Linköping Studies in Science and Technology

Dissertation No. 2094

\title{
Tailoring Conducting \\ Polymer Interface for \\ Sensing and Biosensing
}

Lingyin Meng
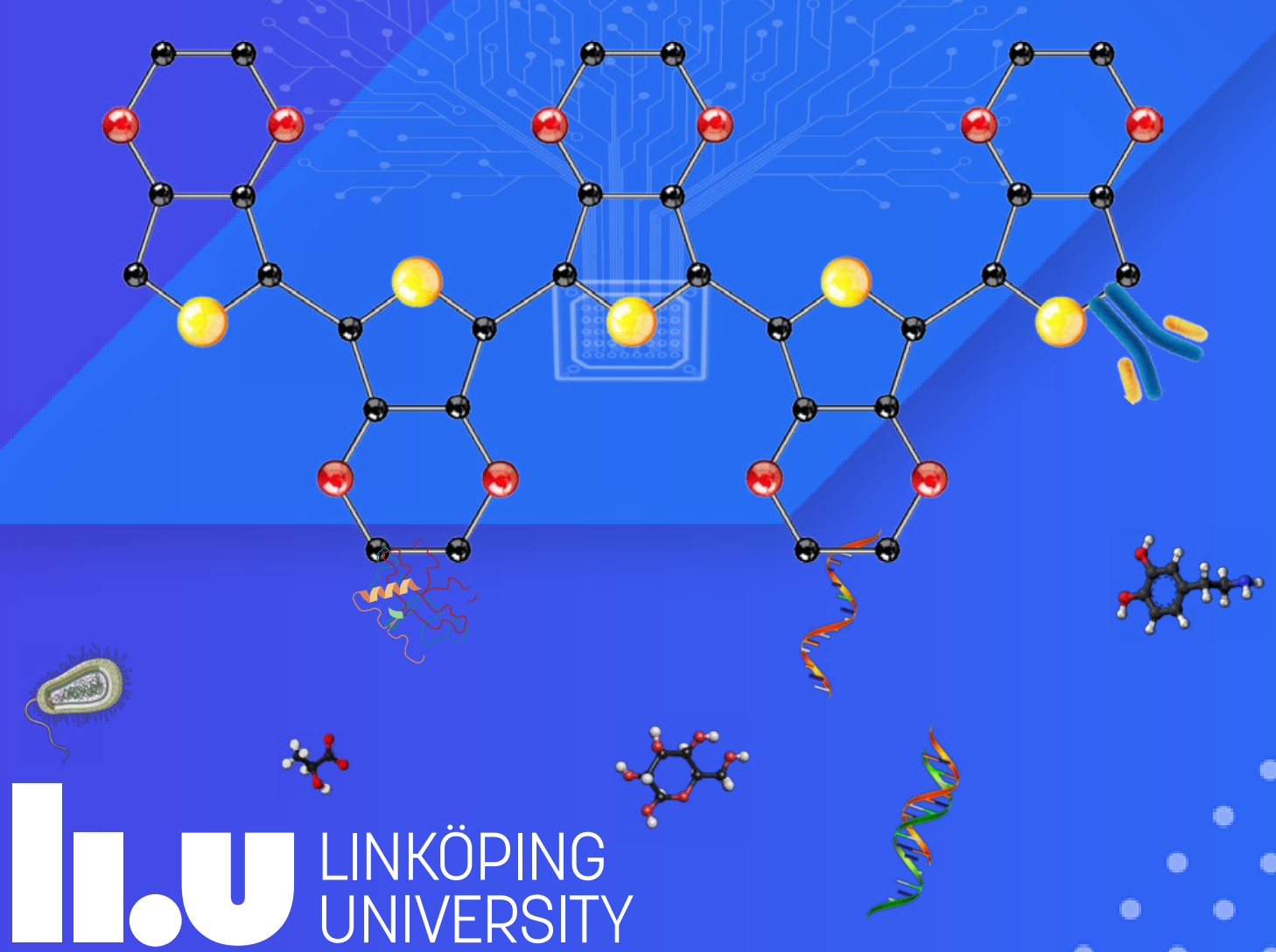
Linkoping Studies in Science and Technology.

Dissertations No. 2094

\title{
Tailoring Conducting Polymer Interface for Sensing and Biosensing
}

\author{
Lingyin Meng
}

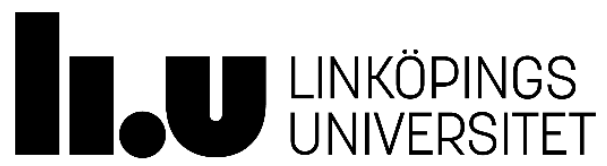

Biosensors and Bioelectronics Unit

Division of Sensor and Actuator Systems (SAS)

Department of Physics, Chemistry and Biology (IFM)

Linköping University, SE-581 83 Linköping, Sweden

Linköping 2020 
During the course of the research underlying this thesis, Lingyin Meng was enrolled in Forum Scientium, a multidisciplinary doctoral program at Linköping University, Sweden

Copyright (C) Lingyin Meng 2020

Cover, PEDOT as electronic interface for sensing and biosensing, designed by Lingyin Meng

Printed in Sweden by Liu-Tryck, Linköping, Sweden, 2020

ISSN: 0345-7524

ISBN: 978-91-7929-800-5 


\section{Abstract}

The routine measurement of significant physiological and biochemical parameters has become increasingly important for health monitoring especially in the cases of elderly people, infants, patients with chronic diseases, athletes and soldiers etc. Monitoring is used to assess both physical fitness level and for disease diagnosis and treatment. Considerable attention has been paid to electrochemical sensors and biosensors as pointof-care diagnostic devices for healthcare management because of their fast response, low-cost, high specificity and ease of operation. The analytical performance of such devices is significantly driven by the high-quality sensing interface, involving signal transduction at the transducer interface and efficient coupling of biomolecules at the transducer bio-interface for specific analyte recognition. The discovery of functional and structured materials, such as metallic and carbon nanomaterials (e.g. gold and graphene), has facilitated the construction of high-performance transducer interfaces which benefit from their unique physicochemical properties. Further exploration of advanced materials remains highly attractive to achieve well-designed and tailored interfaces for electrochemical sensing and biosensing driven by the emerging needs and demands of the "Internet of Things" and wearable sensors.

Conducting polymers (CPs) are emerging functional polymers with extraordinary redox reversibility, electronic/ionic conductivity and mechanical properties, and show considerable potential as a transducer material in sensing and biosensing. While the intrinsic electrocatalytic property of the CPs is limited, especially for the bulk polymer, tailoring of CPs with controlled structure and efficient dopants could improve the electrochemical performance of a transducer interface by delivering a larger surface area and enhanced electrocatalytic property. In addition, the rich synthetic chemistry of CPs endows them with versatile functional groups to modulate the interfacial properties of the polymer for effective biomolecule coupling, thus bridging organic electronics and bioelectrochemistry. Moreover, the soft-material characteristics of CPs enable their use for the development of flexible and wearable sensing platforms which are inexpensive and light-weight, compared to conventional rigid materials, such as carbons, metals and semiconductors.

This thesis focuses on the exploration of CPs for electrochemical sensing and biosensing with improved sensitivity, selectivity and stability by tailoring CP interfaces at different levels, including the $\mathrm{CP}$-based transduction interface, $\mathrm{CP}$-based bio-interface and $\mathrm{CP}$ based device interface. 
First, we demonstrate different strategies for tailoring the physicochemical properties of poly (3,4-ethylenedioxythiophene) (PEDOT) beyond its intrinsic properties, via charge effects, structural effects and by the use of hybrid materials, as a CP-based transduction interface to improve sensing performance of various analytes. 1) A positively-charged PEDOT interface, and a negatively-charged carboxylic-acid-functionalised PEDOT (PEDOT:COOH) interface were developed to modulate the electrode kinetics for oppositely-charged analytes, e.g. negatively-charged nicotinamide adenine dinucleotide (NADH) and positively-charged dopamine (DA), respectively. These interfaces displayed high sensitivity and wide linear range towards the analytes due to the electrostatic attraction effect. 2) Various structured PEDOT including porous microspheres and nanofibres were synthesised via hard-template and soft-template methods, respectively, and were employed as building blocks for a hierarchical PEDOT and 3D nanofibrous PEDOT transduction interface, that facilitated signal transduction for NADH. 3) A PEDOT hybrid material interface was developed via using a novel bifunctional graphene oxide derivative with high reduction degree and negatively-charged sulphonate terminal functionality (S-RGO) as dopant to create PEDOT:S-RGO which delivered an enhanced electrochemical performance for various analytes.

Based on the established CP-based transduction interface, biomolecules (e.g. enzymes) could be coupled to the $\mathrm{CP}$ surface to create $\mathrm{CP}$-based bio-interfaces for biosensing. The immobilisation of enzyme was realised via either covalent bonding to a PEDOT derivative bearing a $-\mathrm{COOH}$ group (PEDOT-COOH) through EDC/NHS chemistry, or by physical absorption into the $3 \mathrm{D}$ porous PEDOT structure. The CP-based biointerfaces were used to demonstrate the stable immobilisation of two different types of enzymes, i.e. lactate dehydrogenase and lactate oxidase, achieving the biosensing of analytes by relay bioelectrochemical signal transduction.

Together, $\mathrm{CP}$ was employed as the $\mathrm{CP}$-based device interface for the fabrication of a flexible and wearable biosensing device. A 3D honeycomb-structured graphene network was generated in-situ on a flexible polyimide surface by mask-free patterning using laser irradiation. The substrate was then reinforced with PEDOT as a polymeric binder to stabilise the $3 \mathrm{D}$ porous network by adhesion and binding, thus minimising the delamination of the biosensing interface under deformation and enhancing the mechanical behaviours for use in flexible and wearable devices. The subsequent nanoscale-coating of Prussian blue and immobilisation of enzyme into the 3D porous network provided a flexible platform for wearable electrochemical biosensors to detect lactate in sweat.

Keywords: conducting polymer, interface, sensing, biosensing, bioelectronics 


\section{Populärvetenskaplig Sammanfattning}

Rutinmässig övervakning av hälsorelaterade fysiologiska och biokemiska parametrar har blivit allt viktigare för ett stort antal människor bland annat seniorer, spädbarn, patienter med kroniska sjukdomar, idrottare, soldater och med flera, på både en fysisk nivå för förebyggande av sjukdomar samt på en medicinsk nivå för diagnos och behandling av sjukdomar. Stor uppmärksamhet har lagts på utveckling av elektrokemiska sensorer och biosensorer som point-of-care (PoC) diagnostiska enheter för rutinmässig sjukvårdsledning genom deras snabba svar, låga kostnad, höga specificitet och enkla drift. Deras analytiska funktioner drivs av avkänningsgränssnittet vilket involverar signaltransduktion vid transducer-gränssnittet och effektiv koppling av biomolekyler till transducer-biogränssnittet för specifik analytigenkänning. Upptäckten av konventionella funktionella och strukturerade material, t.ex. metalliska nanopartiklar, kolnanorör och grafen, har underlättat konstruktionen av transducergränssnitt med hög prestanda på grund av deras unika fysiokemiska egenskaper. Ytterligare forskning av avancerade material är önskvärt för att uppnå ett väldesignat och skräddarsytt gränsnitt för elektrokemisk avkänning och biosensering för Internet of Things och klädd sensorer.

Ledande polymerer (LP) är en typ av nya funktionella polymerer med extraordinär redoxomvändbarhet, elektronisk/jonisk ledningsförmåga och mekaniska egenskaper, som uppvisar betydande potential som ett givarmaterial vid avkänning och biosensering. Medan de inneboende elektrokatalytiska egenskaperna i LP: er är begränsade, speciellt för den skrymmande polymeren, kan skräddarsydda LP: er med kontrollerad struktur och effektiva dopmedel förbättra den elektrokemiska prestandan hos ett givargränssnitt med större ytarea och förbättrade elektrokatalytiska egenskaper. Dessutom ger den syntetiska kemin LP: er mångsidiga funktionella grupper för att modulera gränssnittsegenskaperna för LP: er för att förbättra selektivitet för analytdetektering, såväl som för effektiv biomolekylkoppling som ett biogränssnitt som överbryggar den organiska elektroniken och det biologiska system som stöds av de LP: s organkemiska natur. Dessutom möjliggör de mjuka materialegenskaperna för LP: er för användning i utveckling av en flexibla och bärbara avkänningsplattformar med låg kostnad och lätt vikt, jämfört med konventionella styva material, såsom metaller och halvledare.

Denna avhandling fokuserar på utforskning av LP: er för elektrokemisk avkänning och biosensering med förbättrad känslighet, selektivitet och stabilitet genom att skräddarsy LP: s gränssnitt i olika nivåer, inklusive LP-baserat transduktionsgränssnitt, LP-baserat bio-gränssnitt och LP-baserat enhetsgränssnitt. 
Först demonstrerar vi olika strategier för att skräddarsy fysikalisk-kemiska egenskaper hos poly (3,4-etylendioxytiofen) (PEDOT) som ett LP-baserat transduktionsgränssnitt för avkänning via laddningseffekter, struktureffekter och hybridmaterialeffekter för förbättrad prestanda för olika analyser utöver dess inre egenskaper. 1) Ett positivt laddat hierarkiskt PEDOT-gränssnitt och ett negativt laddat karboxylsyra-funktionaliserad PEDOT (PEDOT: COOH) gränssnitt utvecklades för att modulera gränssnittets kinetik för de motsatt laddade analyterna, t.ex. negativt laddad ß-Nicotinamidadeninudukleotid (NADH) respektive positivt laddat dopamin (DA). Den elektrokemiska avkänningsprestandan hos dessa analyser förbättrades baserat på laddningseffekten med högre känslighet och ett bredare linjärt intervall. 2) Med tanke på den väl skrymmande filmbildande egenskapen och den resulterande låga tillgängliga aktiva ytan för PEDOT, syntetiserades olika strukturerade PEDOT inklusive porösa mikrosfärer och nanofibrer via en hård mall respektive en mjuk mall och användes sedan som byggstenar för hierarkiska PEDOT och 3D nanofibrösa PEDOT-transduktionsgränssnitt, vilket underlättar signaltransduktion för NADH. 3) Ett LP-hybridmaterialgränssnitt utvecklades med användning av ett nytt bi-funktionellt grafenoxidderivat med hög reduktionsgrad och negativt laddad sulfonatterminal funktionalitet (S-RGO) med förbättrad elektrokemisk prestanda för olika analyser.

Baserat på det etablerade LP-baserade transduktionsgränssnittet utvecklades sedan de LP-baserade bio-gränssnitten med immobilisering av biomolekyler (t.ex. enzym) för biosensering. Immobiliseringen av enzym på LP-gränssnittet realiserades via antingen kovalent bindning till PEDOT-derivatbärande -COOH-grupper (PEDOT-COOH) genom EDC/NHS-kemi eller fysisk absorption i porösa 3D-PEDOT-strukturer. De LPbiobaserade gränssnitten visar stabil immobilisering av två olika typer av enzymer, d.v.s. laktatdehydrogenas och laktatoxidas, vilket uppnår biosensering av analyter genom en successiv bioelektrokemisk signaltransduktion.

Tillsammans användes LP: er som det LP-baserade enhetsgränssnittet för tillverkning av en flexibel och bärbar biosenseringsanordning. Ett tredimensionellt bikakestrukturerat grafennätverk genererades in-situ på den flexibla polyimidytan genom maskfri mönstring med laserbestrålningsteknik. Substratet förstärktes sedan med nanodeponerat PEDOT som ett polymert bindemedel för att stabilisera det porösa 3Dnätverket genom vidhäftning och bindning, vilket sålunda förbättrade det mekaniska beteendet för flexibla och bärbara anordningar. Den sekventiella beläggningen på nanoskala av Preussiskt blått (PB) och immobiliseringen av enzym i det porösa 3Dnätverket minimerade delaminering av biosenseringsgränssnittet vid deformation, vilket försedde en flexibel plattform för en bärbar elektrokemisk biosensor för detektering av laktat i svett med det monterade treelektrodsystemet. 


\section{Acknowledgement}

It has been an interesting study experience as a $\mathrm{PhD}$ student at Biosensors and Bioelectronics Unit, Division of Sensor and Actuator Systems (SAS), Department of Physics, Chemistry and Biology (IFM) at Linköping University during the last four years. All the research works included in the thesis could not be accomplished without the help and support from all the people who I worked with. Beyond the thesis, I also learned from them to be more independent, to communicate, to share and to help in scientific research. Here, I would like to express my sincere gratitude to them.

First and foremost, I would like to thank my supervisor Assoc. Prof. Wing Cheung Mak, who is the group leader for Biosensors and Bioelectronics Unit. I am very grateful for his great patient guidance and encouragement for me to carry out the research works. I really admire his innovative thinking in research. Thanks to his insightful comments and suggestions during each group and individual meeting. More importantly, it is his optimistic attitude towards stress and difficulties encouraged me to be strong and positive in daily life. Special thanks goes to his understanding and support during the rock bottom in my life.

I also would like to express my sincere gratitude to my supervisor Prof. Anthony P.F. Turner, who was the head of Biosensors and Bioelectronics Centre. Thank you for taking me as a PhD student at Linköping University. It is a good memory to work together with you. I really enjoy the discussion and thanks to your scientific comments and thought-provoking suggestions during each group and individual meeting. Wish you a happy $70^{\text {th }}$ birthday and wish you a happy retired life.

My sincere thanks also go to my co-supervisor Prof. Daniel Filippini, the head of Sensor and Actuator Systems (SAS), for his sharing of knowledge in optical devices, and cosupervisor Dr. Mikhail Vagin from Laboratory of Organic Electronics (LOE), Department of Science and Technology (ITN), Norrköping, Linköping University for his sharing of knowledge in electrochemistry.

My $\mathrm{PhD}$ study is partially financed by China Scholarship Council (CSC), who provides support for international academic study abroad. I thank my previous supervisor Prof. Yunsong Zhang at Sichuan Agricultural University during my master study period for his encouragement to pursue my $\mathrm{PhD}$ study abroad and also his concern during the last four years.

I am very grateful for all the staff in Kardiologiska kliniken and Thorax-kärlkliniken in Universitetssjukhuset. 
I would like to thank all the previous and current labmates Dr. Tawatchai Kangkamano, Miss Manisa Kaewsem, Mr. Sinan Uzuncar, Dr. Junya Tsutsumi, Mr. Sebastian Thierry, Mr. Phachara Suklim, Miss Frida Dagsgård, Dr. Gerardo Zambrano, Mr. Kiattisak Promsuwan, Miss Supatinee Kongkaew, Mr William Wijård and Miss Viktoria Hell to work together in Biosensors and Bioelectronics group and to have afterwork drinks and fun parties with nice international food. Thanks to my friends Manav Tyagi, Robert Pilstål, Ligen Hu, Weiping Wu, Lianlian Liu, Lei Wang, Yingzhi Jin, Yanfeng Liu, Chi Xiao, Jiwen Hu, Feng Wang, Bin Zhang, Fuxiang Ji, Zhixing Wu, Xin Zhang, Yusheng Yuan, Rui Shu, Rui Zhang and Leiqiang Qin for your continuous friendship.

My gratitude also goes to Stefan Welin Klintström for offering me the opportunity to be a member in Forum Scientium, and Anette Anderson for her help in administrative issues.

Last but not the least, I would like to thank all my families for their endless support. I am sincerely grateful for my mother to help to take care of my little daughter. And for my daughter, An'an, Happy 3rd Birthday. Your innocent smile is the most powerful weapon to defeat my bad mood. I wish you a carefree childhood. Special thanks to my wife Danfeng for your love and support.

Linköping

June 2020 


\section{List of Publications}

\section{Publications included in the thesis}

The $\mathrm{PhD}$ thesis is based on the following publications:

\section{Research paper:}

1) Positively-charged hierarchical PEDOT interface with enhanced electrode kinetics for NADH-based biosensors

Lingyin Meng, Anthony PF Turner, and Wing Cheung Mak.

Biosensors and Bioelectronics 2018, 120, 115-121.

Contribution: Partly conceived the experimental design, performed all the experiments and data analysis, wrote the manuscript and partly revised the manuscript.

2) Modulating electrode kinetics for discrimination of dopamine by a PEDOT:COOH interface doped with negatively charged tricarboxylate

Lingyin Meng, Anthony PF Turner, and Wing Cheung Mak.

ACS Applied Materials \& Interfaces 2019, 11 (37), 34497-34506.

Contribution: Partly conceived the experimental design, performed all the experiments and data analysis, wrote the manuscript and partly revised the manuscript.

3) Bi-functional sulphonate-coupled reduced graphene oxide as an efficient dopant for a conducting polymer with enhanced electrochemical performance

Lingyin Meng, Frida Dagsgård, Anthony P. F. Turner, and Wing Cheung Mak

Journal of Materials Chemistry C, 2020: DOI: 10.1039/D0TC02402C.

Contribution: Conceived the experimental design, performed all the characterisations and data analysis, partly performed the measurements, wrote the manuscript and partly revised the manuscript.

4) Tunable 3D nanofibrous and bio-functionalised PEDOT network explored as a conducting polymer-based biosensor.

Lingyin Meng, Anthony PF Turner, and Wing Cheung Mak

Biosensors and Bioelectronics, 2020, 159, 112181.

Contribution: Partly conceived the experimental design, performed all the experiments and data analysis, wrote the manuscript and partly revised the manuscript.

5) Conducting polymeric binder reinforced graphene network for 3D porous enzymatic electrodes and their application in wearable lactate biosensor

Lingyin Meng, Anthony PF Turner, and Wing Cheung Mak

In Manuscript 
Contribution: Partly conceived the experimental design, performed all the experiments and the data analysis, wrote the manuscript.

\section{Review paper:}

1) Soft and flexible material-based affinity sensors

Lingyin Meng, Anthony PF Turner, and Wing Cheung Mak.

Biotechnology Advances, 2020, 39, 107398.

Contribution: Partly wrote the manuscript and partly revised the manuscript. 


\section{Publications not included in the thesis:}

1) Product-to-intermediate relay achieving complete oxygen reduction reaction (cORR) with Prussian blue integrated nanoporous polymer cathode in fuel cells

Tawatchai Kangkamano, Mikhail Vagin, Lingyin Meng, Panote Thavarungkul, Proespichaya Kanatharana, Xavier Crispin, Wing Cheung Mak.

Nano Energy, 2020, 78, 105125

Contribution: performed and wrote the experimental and discussion section for the XPS and FTIR measurement, data interpretation and analysis.

2) Bio-PEDOT: modulating carboxyl moieties in poly(3,4-ethylenedioxythiophene) for enzyme-coupled bioelectronic interfaces

Kiattisak Promsuwan, Lingyin Meng, Phachara Suklim, Warakorn Limbut, Panote Thavarungkul, Proespichaya Kanatharana, and Wing Cheung Mak

ACS applied materials \& interfaces, DOI: 10.1021/acsami.0c10270

Contribution: Partly conceived the experimental design, partly performed the electrochemical measurements, performed all the electron microscopy and spectrum characterisations, partly analyse the data, partly wrote the manuscript and partly revised the manuscript.

3) Processable and nanofibrous polyaniline:polystyrene-sulfonate (nano-PANI:PSS) for the fabrication of catalyst-free ammonium sensors and enzyme-coupled urea biosensors

Sinan Uzuncar, Lingyin Meng, Anthony P.F. Turner, Wing Cheung Mak.

\section{Submitted}

Contribution: Performed all the electron microscopy and spectrum characterisations, partly performed the electrochemical measurements, partly analyse the data, partly wrote the manuscript and partly revised the manuscript.

4) Evaluation of intrinsic physio-electrochemical properties of allotropic carbon-based papers for flexible electrode platform

Supatinee Kongkaew, Lingyin Meng, Warakorn Limbuta, Proespichaya Kanatharan, Panote Thavarungkul, Wing Cheung Mak

In manuscript

Contribution: Performed all the electron microscopy and spectrum characterisations, partly analyse the data, partly performed the application measurements for glucose detection and capacitor, partly revised the manuscript. 


\section{Abbreviations}

Acetonitrile

$\mathrm{ACN}$

Ascorbic acid

AA

$\beta$-Nicotinamide adenine dinucleotide

$\mathrm{NADH}$

Carbon nanotubes

CNTs

Charge-transfer resistance

Ret

Chemically modified electrodes

CMEs

Colloidal microparticles

CMs

Conducting polymers

CPs

Cyclic voltammetry

CV

Deoxyribonucleic acid

DNA

Direct electron transfer

DET

Differential pulse voltammetry

DPV

Dopamine

DA

Electrocardiograph

ECG

Electrochemical Impedance Spectroscopy

EIS

Ethyl(dimethylaminopropyl) carbodiimide

EDC

Flavin adenine dinucleotide

FAD

Glassy carbon electrode

GCE

Glucose oxidase

$\mathrm{GOx}$

Graphene oxide

GO

Indium-tin-oxide

ITO

Internet of Things

IoT

In-vitro diagnostics

IVDs

Lactate dehydrogenase

LDH

Lactate oxidase

LOx

Layer-by-layer

LBL

Linear sweep voltammetry

LSV

Limit of detection

LoD

Limit of quantification

LoQ

N-hydroxysuccinimide

NHS

N-hydroxysulfosuccinimide

S-NHS

Organic bioelectronics

OBEs

Organic electrochemical transistor

OECT

Organic field-effect transistor

OFET

Polyaniline

PANI

Polydimethylsiloxane

PDMS

Polyethylene terephthalate

PET

$\mathrm{xi}$ 
Polyimide

Poly(methylmethacrylate)

Polypyrrole

Poly(styrenesulfonate)

Poly(3,4-etylendioxytiofen)

Prussian blue

Reduced graphene oxide

Sulphonate-coupled reduced graphene oxide

Tetra-butylammonium perchlorate

Three-dimensional

Uric acid
PI

PMMA

PPy

PSS

PEDOT

PB

RGO

S-RGO

TBAP

3D

UA 


\section{Table of Contents}

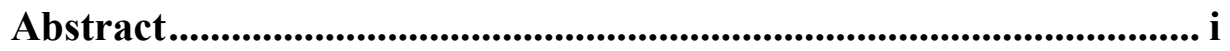

Populärvetenskaplig Sammanfattning ............................................ iii

Acknowledgement .................................................................................... v

List of Publications........................................................................... vii

Abbreviations ................................................................................. xi

Chapter 1 Introduction.................................................................... 1

1.1 Sensors and Biosensors for Health Monitoring ........................... 1

1.2 Soft and Flexible Materials ......................................................... 2

1.3 Aim and Outline of the Thesis ................................................ 3

Chapter 2 Bioelectronics .............................................................................. 5

2.1 Development of Bioelectronics................................................. 5

2.2 Information from Biological System ....................................... 9

2.3 Organic Bioelectronics.......................................................... 12

Chapter 3 Sensors and Biosensors in Bioelectronics ........................ 15

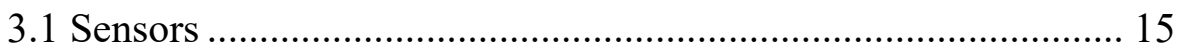

3.1.1 Sensor Definition ...................................................... 15

3.1.2 Sensor Criteria............................................................ 16

3.1.3 Sensor Classification .................................................. 17

3.2 Electrochemical Sensors ....................................................... 18

3.2.1 Electrochemical Techniques ........................................ 18

3.2.2 Chemically Modified Electrodes................................... 22

3.2.3 Electrochemical Sensors for Healthcare Monitoring ....... 25

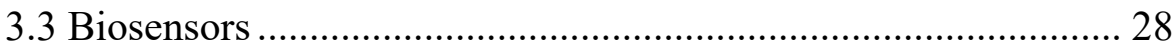

3.3.1 Biosensor Principle .................................................... 28

3.3.2 Biosensor Classification ................................................ 29

3.4 Electrochemical Enzyme-based Biosensors............................... 30

3.4.1 Generations of Enzyme-based Biosensors ..................... 30

3.4.2 Immobilisation of Enzymes ......................................... 32

3.4.3 Electrochemical Enzyme-based Lactate Biosensors........ 33 
3.4.4 Electrochemical Enzyme-based Glucose Biosensors ....... 34

Chapter 4 Conducting Polymers........................................................... 37

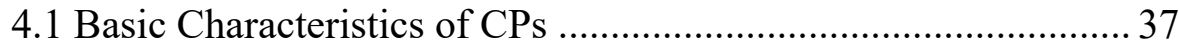

4.1.1 Development of CPs..................................................... 37

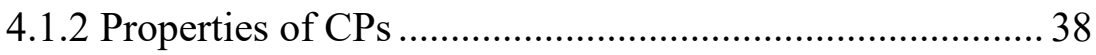

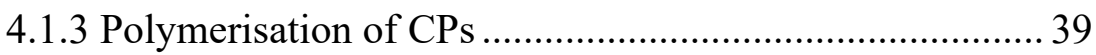

4.1.4 Applications of CPs ....................................................... 41

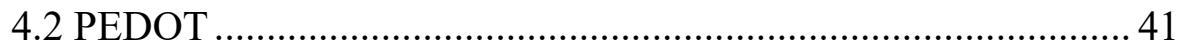

4.2.1 Incorporation of Dopant into PEDOT .............................. 41

4.2.2 Modulating PEDOT Surface Charge in Sensing and

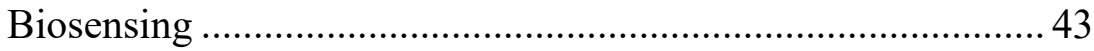

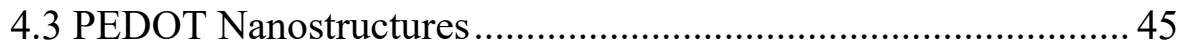

4.3.1 Nanostructure Features ...................................................... 45

4.3.2 PEDOT Nanostructures ............................................... 45

4.3.3 Nanostructured Interface in Sensing and Biosensing ....... 49

4.4 PEDOT Derivatives................................................................ 50

4.4.1 PEDOT Derivative Properties ....................................... 50

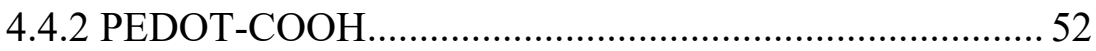

4.4.3 Biofunctionalisation of PEDOT-COOH......................... 52

4.5 PEDOT Composites ................................................................. 54

4.5.1 Pristine PEDOT .......................................................... 54

4.5.2 PEDOT Composites in Sensing and Biosensing ............. 55

Chapter 5 Polymer-based Flexible and Wearable Bioelectronics .. 59

5.1 Flexible and Wearable Bioelectronics........................................ 59

5.2 Soft and Flexible Materials .......................................................... 61

5.3 All-polymer based Bioelectronics .............................................. 64

Chapter 6 Summary of the Papers Included ....................................... 65

Chapter 7 Conclusion and Outlook ................................................... 75

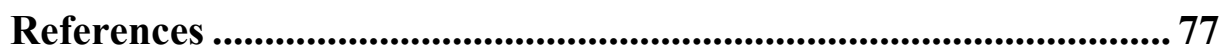




\section{Chapter 1 Introduction}

\subsection{Sensors and Biosensors for Health Monitoring}

Traditionally, people were used to visiting doctors for diagnosis and treatment only when they were sick. Over the past few decades, the significant increase in the quality of our daily life promoted more frequent regular check-ups, which are favourable for the early diagnosis and treatment of diseases, thus reducing the suffering of patients and national healthcare costs, especially for the chronic diseases that are the leading causes of death and disability worldwide including cardiovascular diseases and diabetes ${ }^{1}$. This diagnosis has generally relied on complicated, expensive and time-consuming laboratory devices, which perform the medical tests on samples (e.g. blood and tissue) taken from human body invasively in central hospitals.

Nowadays, there is increasing demand for healthcare services from seniors, infants, patients with chronic diseases, athletes and soldiers etc. To address this, health selfmonitoring is playing a vital role as an effective solution to track an individual's physiological and biochemical information in decentralised locations to assess the physical fitness levels for health management and, at a medical level, for disease treatment. A variety of home-based sensors and biosensors have been developed for point-of-care (PoC) tests by the research community and industry. Among these, electrochemical sensors and biosensors have gained the dominant role in home-based devices for health monitoring due to their ease of operation, low-cost, portability and fast response. Notable examples include the portable electrocardiogram (ECG) monitor and the most successful commercialised biosensor of all, glucometer for blood glucose measurement for the management of diabetes ( $>5 \%$ of the world market for biosensors) $)^{2}$.

Given the limitations imposed by invasiveness of conventional home-based devices and the demand for continuous monitoring in daily life, researchers are working on nextgeneration bioelectronic devices which move away from home-based PoC tests towards minimally/non-invasive flexible and wearable sensors for continuous and real-time personalised monitoring. Driven by this, continuous glucose monitoring (CGM) can now provide real-time and long-term measurements of body glucose levels, which also can be combined with insulin pumps to form an automated feedback loop to manage insulin delivery when hypo/hyperglycemia is developing. Beyond this, many other efforts have been devoted to the development of flexible and wearable electronic devices for healthcare management, in which an important prerequisite to be fulfilled is a low- 
cost, light-weight, high flexible and high-quality sensing and biosensing platform. The integration and miniaturisation of these with the "Internet of Things (IoT)" promises the best usage of data for widespread health monitoring in the future.

\subsection{Soft and Flexible Materials}

Recent advancements in soft and flexible materials are playing a key role in propelling the field of soft electronics and new types of bioelectronics, and their subsequent application in wearable and flexible sensors and biosensors. The crucial issue for conventional rigid materials, such as metals, silicon compounds and ceramics, in soft electronics and bioelectronics is their low compatibility and the mechanical mismatch (e.g. flexibility and stretchability) with surrounding tissues (e.g. skin and cornea) at the target site $^{3}$. Compared to the traditional rigid materials, soft and flexible materials consisting of synthetic polymers, colloids, gels, biomaterials etc., have attracted extensive attention for various applications including microfluidic devices, electronic skins, soft robotics and actuators, wearable sensors and biosensors ${ }^{4}$. Soft and flexible materials possess unique mechanical characteristics, facilitating flexibility under large deformation including bending, folding and stretching, which can be potentially employed at the fundamental device level as substrates and encapsulation layers. Besides, the versatile features of soft and flexible materials can be tailored with various structures and new physicochemical properties to provide active sensing and biosensing components.

Among the soft and flexible materials, conducting polymers (CPs) have attracted tremendous interest and have developed rapidly in both the academic and industrial communities. Desirable organic polymer characteristics such as well-maintained flexibility and structural diversity, and newly introduced electronic/ionic conductivity and redox reversibility by a doping/de-doping process, make them well suited for applications in electrochemistry-related applications, such as energy and biomedical devices $^{5}$. In addition, the similarities of CPs in their organic nature with biological molecules facilitates the concept of organic bioelectronics (OBEs), bridging the world of organic electronics in CPs with bioelectronics in biological systems ${ }^{6}$. OBEs comprising of CPs as the communication interface have demonstrated promising capabilities for various biomedical fields, such as tissue scaffolds ${ }^{7}$, neural interfaces ${ }^{8}$, drug delivery ${ }^{9}$, artificial muscles ${ }^{10}$, and sensors and biosensors ${ }^{11}$. In sensing and biosensing applications, CPs can be utilised as the transduction interface relying on their electronic/ionic conductivity. On the other hand, CPs can serve as a sensing element interface by further functionalisation with specific biorecognition ability by virtue of their rich synthetic chemistry and conjugation. To achieve high sensitivity and good 
selectivity using CPs as the communication interface for OBEs, tailoring the CP's interface to deliver enhanced electrochemical activity for transduction and functionality for bioconjugation is essential.

\subsection{Aim and Outline of the Thesis}

The analytical performance of electrochemical sensors and biosensors in the form of $\mathrm{PoC}$ or flexible devices for health monitoring is dependent on high-quality sensing interfaces through either signal transduction or specific analyte recognition. The evolution of conventional functional and structured materials, such as metallic and carbon nanomaterials (e.g. gold and graphene), has facilitated the construction of highperformance transducer interfaces by virtue of their unique physicochemical properties. Further exploration of advanced materials in the field of soft and flexible materials remains highly attractive for electrochemical sensing and biosensing driven by the emerging needs of the IoT and wearable sensors.

Building upon the scientific background mentioned above, this thesis aims to explore the application of CPs for electrochemical sensing and biosensing by tailoring the CP interface with specific physicochemical characteristics to deliver fast electrochemical kinetics and high analytical performance with improved sensitivity, selectivity for analytes together with better stability. The tailoring of the physicochemical properties of CPs is achieved from three different aspects, including:

1) tailoring the $\mathrm{CP}$-based transduction interface for chemical sensing via modulating the charge effect, morphology effect and composite effect;

2) tailoring the CP-based bio-interface for stable immobilisation of biorecognition elements for biosensing;

3) tailoring CP-based device interface for flexible bioelectronics.

The thesis is composed of 7 Chapters. In Chapter 1, introductory information about "sensors and biosensors for health monitoring" and "soft and flexible materials" are given. In Chapter 2, a brief review is provided on the development of the "bioelectronics" field from the aspects of bioelectricity, biomarker, materials and biomaterials, followed by a description of hierarchical "biological system" that can be used for health monitoring and disease diagnosis. The new era of "organic bioelectronics" which bridges the gap at the biotic/abiotic interface between biological systems and electronics is also discussed. Chapter 3 discusses the function and application of electrochemical sensors and biosensors in the field of bioelectronics with detailed descriptions of the sensing and biosensing definition, structure and mechanism, electrode modification and immobilisation, signal transduction of several key biomarkers. Chapter 4 is dedicated to exploring the application of CPs in sensing and 
biosensing. The basic characteristics of CPs are covered first in the context of their development, properties, polymerisation techniques and applications. PEDOT, as the most commonly used $\mathrm{CP}$, is then the focus and is discussed as a sensing and biosensing interface by tailoring its physicochemical properties in the respect of surface charge, nanostructure, derivatives and composites. The main results of the tailoring of PEDOT for sensing biosensing are presented in Paper I-V. In Chapter 5, the advancement of soft and flexible materials and their application for the fabrication of next-generation of flexible and wearable bioelectronic devices are discussed. Paper $\mathrm{V}$ and the review paper included in this thesis present the successful employment of soft and flexible material in the fabrication of sensors and biosensors. Details of the contributions of our work to the field of sensing and biosensing based on CPs are summarised in Chapter 6. Finally, the conclusion and future perspectives are given in Chapter 7 . 


\section{Chapter 2 Bioelectronics}

\subsection{Development of Bioelectronics}

Bioelectronics, specifically bio-molecular electronics, is a developing interdisciplinary research area studying the interactions between biological entities and electronic devices through electrical changes, or as defined by the National Institute of Standards and Technology (NIST) in the report in 2009, is the discipline resulting from the convergence of biology and electronics ${ }^{12}$. The bioelectronic field encompasses a range of topics at the interface of biology and electronics in various areas, such as the study of bioelectricity, detection of physiological signals and biomedical information via biomarkers, the development of materials and biomaterials and so on ${ }^{12}$. A brief timeline of some selected events in the development of bioelectronics is described in Figure 2.1.

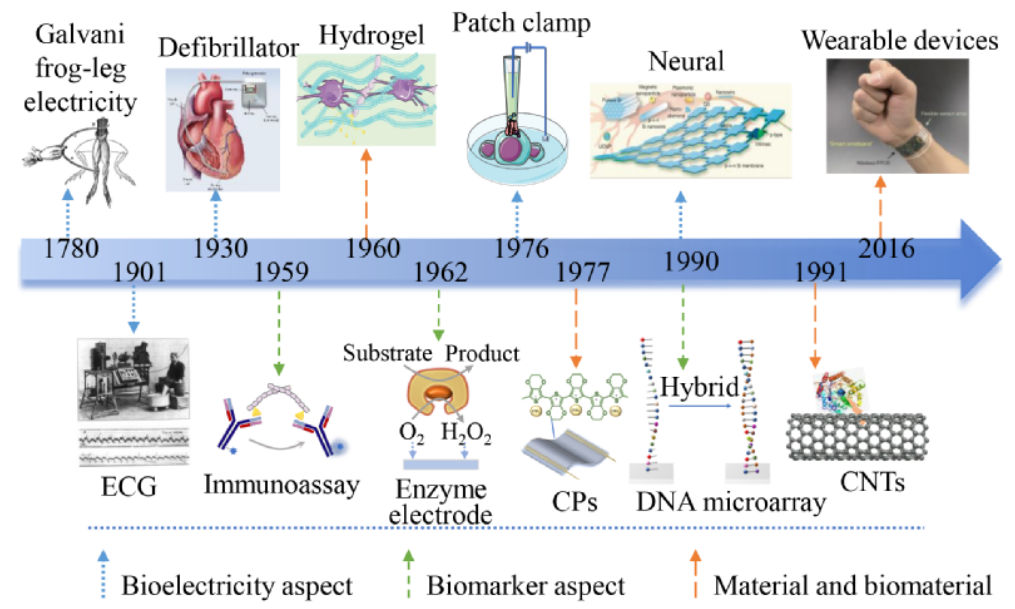

Figure 2.1 Brief timeline of the selected events in the development of bioelectronics. Parts of the insets are adapted with permission from Ledesma, H. A. et al. (neural interface) ${ }^{13}$; Gao, W. et al. (wearable devices) ${ }^{14}$.

Research in the area of bioelectronics can be traced back to the $18^{\text {th }}$ century with Luigi Galvani's discovery that the muscles of a dead frog's leg twitched when struck by an electrical spark, and this initiated the study of bioelectricity from tissues. Since then, much effort has been devoted over the last century to the research and development of bioelectronics for healthcare and medicine. At the beginning of 1901, Willem Einthoven initially evolved the string galvanometer ECG to record the electrical activity of the 
heart and received the Nobel Prize in 1924 for his contribution of the first practical system of ECG, which facilitated the establishment of cardiology and is now an integral part of clinical diagnosis ${ }^{15}$. From the 1930s, external defibrillators and artificial cardiac pacemakers have been developed to rectify life-threatening cardiac arrest and dysrhythmias by delivering a dose of electric current to restore normal heart rhythms This was followed with the appearance of implantable cardioverter defibrillators (ICD) and implantable pacemakers in recent decades in the form of battery-operated devices inside the body. The study of bioelectronics from organ level down to individual isolated living cell level has been realised by the development of patch clamp technique, in 1976, by Erwin Neher and Bert Sakmann who received the Nobel Prize in Physiology or Medicine in $1991^{16}$. The patch clamp technique provided the necessary sensitivity to measure ion channel meditated electrical current flow through the cell membrane of excitable cells including neurons, cardiomyocytes, muscle fibres and so on. Moreover, integrated neural interfaces based on metals, silicon, complementary metal-oxidesemiconductor (CMOS) and CPs have been increasingly explored for bioelectrical signal communication between the neural network and external devices, since the mid$1990 \mathrm{~s}^{17}$, enabling the research on brain function monitoring, diagnosis and therapy for neurological disorders such as Parkinson's disease ${ }^{18}$.

Besides the research and development of bioelectronics in bioelectricity, bioelectronics also established the transduction of physiological signals and biomedical events via the detection of biomarkers. The transduction of biomarkers, including small molecular metabolites (e.g. dopamine, glucose, lactate), antigen and nucleic acid etc., could be realised by incorporating a synthetic receptor or a biological recognition element (e.g. enzyme, antibody and nucleic acid with specific sequence) in an electronic transducer. Yalow and Berson inaugurated the field of bioaffinity monitoring of small molecules from human body by their development of the first immunoassay for insulin in 1959, and Yalow received the Nobel Prize in Medicine in $1977^{19}$. The "enzyme electrode" concept relying on biocatalytic reaction was elucidated by Leyland C. Clark, in 1962, and the first commercial blood glucose analyser (YSI Model 23 Analyzer) based on this was successfully launched in 1975. A further milestone in this important technology was the emergence of a convenient, hand-held commercial format of glucose analyser which revolutionised the use of biosensors in $1987^{2}$. With the discovery of the DNA double helix structure in 1953 by James Watson and Francis Crick (awarded with Nobel Prize in Physiology or Medicine in 1962) and the invention of the polymerase chain reaction (PCR) by Kary Mullis in 1983 (awarded with Nobel Prize in Chemistry in 1993), identifying and sequencing of deoxyribonucleic acid (DNA) molecules have attracted intensive interest and rapidly developed in 1990s. These developments contributed to bioelectronics in the field of DNA biosensors and microarrays, because DNA and ribonucleic acid (RNA) could be sensitive biomarkers for pathogen 
recognition, sub-atomic diagnostics, genomic sequence analysis and cancer diagnostics ${ }^{20}$.

Based on these foundations, the conversion of biomarkers from biological system into readable electronic signals is another important development in the field of bioelectronics. The key aspect here is achieving an effective communication interface between biological systems and micro- and nano-electronics ${ }^{6}$. The advances in material and biomaterial science, nanotechnology and engineering in recent decades have propelled the fabrication of promising communication interfaces for bioelectronic applications including biological event recognition and electronic signal transduction. Three examples of important materials and biomaterials that impacted on the development of bioelectronic interface are introduced here including hydrogels, CPs and carbon nanotubes (CNTs). Bioelectronic interfacing with biological systems used to be very challenging, especially for cell stimulation and neural recording in implantable devices facing skin, brain and heart, due to the high requirements of biocompatibility and stability in face of immune and inflammatory responses. Hydrogels, crosslinked polymer networks with a high water content, emerged as a promising matrix material for bioelectronic interfaces in tissue engineering, biomedicine and actuators. The first application of a hydrogel in biomedicine was reported by Wichterle and Lim in $1960^{21}$. The unique tissue-like mechanical properties and high biocompatibility of hydrogels can minimise the biomechanical mismatch and immune/inflammatory response between the biological system and electronics ${ }^{22}$. Further functionalisation endows hydrogels with versatility in electrical, mechanical, biocompatible and bifunctional properties suitable for a communication interface in bioelectronics. In addition, sharing similarities to biological molecules in their chemical structure, CPs have been used as another promising biointerfacing material to be integrated with biological systems in electroresponsive surfaces and tissue engineering scaffolds ${ }^{6}$. Ever since the discovery of CNTs, in 1991, by Iijima and graphene, in 2004, by Geim and Novoselov, carbon based nanomaterials have been intensively explored as transducer materials for bioelectronic interfaces through "bottom-up" fabrication with chemical modification, hybridisation and further bio-functionalisation of biological molecules ${ }^{23}$. Moreover, the ongoing miniaturisation and integration of devices with mobile communications are leading the revolution in size, durability and cost for the next-generation of wearable and implantable electronic and bioelectronic devices to fulfill the demand for worldwide distribution of decentralised and personalised monitoring and therapy. The recent proofof-concept study by Gao, in 2016, demonstrated this trend to realise integrated wearable sensor arrays on a conformable patch for multiplexed in situ non-invasive perspiration analysis ${ }^{14}$. 
As shown in Figure 2.2a, the $21^{\text {st }}$ century has witnessed the rapid development of bioelectronics with an increasing number in publications according to an analysis from Web of Science Core Collection including the keyword "bioelectronic*". Although the rise from 2000 (27 publications) to 2014 (147 publications) is relatively slow, it is worthy of note that the number of publications has risen dramatically since 2015 , with 442 publications in 2019. Research and development of bioelectronics are fostered by more than 100 disciplines and multidisciplinary fields, such as biology, engineering, chemistry, physics and material science. Figure 2.2b summarises the top ten disciplines that are closely related to bioelectronics according to the distribution analysis of the above publications in different research fields and disciplines. Material science multidisciplinary, nanoscience and nanotechnology, and chemistry multidisciplinary are the leading three disciplines encompassing bioelectronics. Given the great potential of bioelectronics in fundamental research of biological phenomenon and theory and their visionary applications in next-generation of electronic devices, the field of bioelectronics is poised for influencing our daily life in the near future.

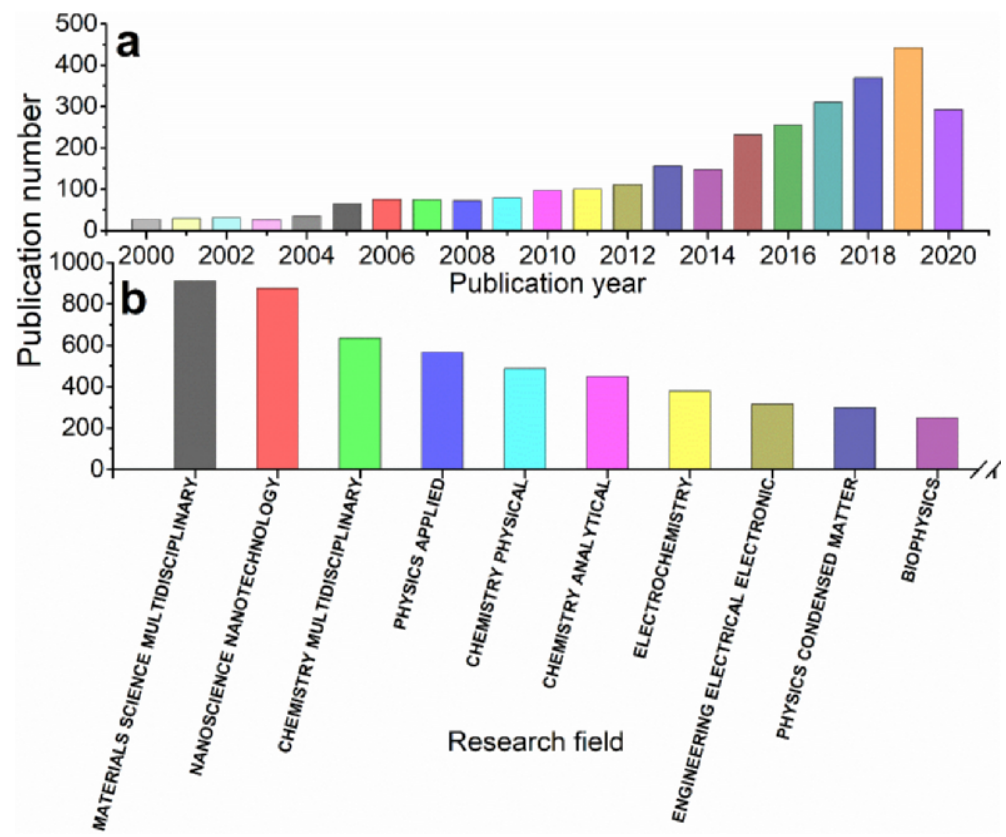

Figure 2.2 Publication number from 2000 to 2020 (until August 24, 2020) and their distribution in different research fields extracted from Web of Science Core Collection using "bioelectronic*" as the topic. 


\subsection{Information from Biological System}

Medical diagnosis has a long history for human beings in the fight against diseases, and it can be traced back to the days of Imhotep in ancient Egypt. The earliest methods applied in different civilisations include the use of empiricism, logic and rationality in diagnosis in ancient Babylonia, four diagnostic methods with inspection, auscultationolfaction, interrogation, and palpation in ancient China, and tests with the body fluids (e.g. urine and sweat) by Hippocrates in ancient Greece. Accurate diagnosis of diseases could not be simply determined from the apparent symptoms by top-down approach from whole-body performance to organ function due to the complexity of the human biological system. Diagnostic tools and techniques for measurement and visualisation, such as the microscope, thermometer and stethoscope, were not used widely until the end of $19^{\text {th }}$ century ${ }^{24}$. Nowadays, the emergence of more sophisticated quantitative diagnosis (e.g. PCR, magnetic resonance imaging (MRI) and gas chromatography-mass spectrometry (GC-MS) etc.) can determine the indicators of disease from the biological system in hierarchical levels in a bottom-up approach from molecular, cellular, tissue and organ to whole-body level.

Figure 2.3 illustrates the hierarchical levels of a biological system. An organism, i.e. the whole-body, is highly organised and structured in a hierarchy that can be disassembled by a top-down direction from large-to-small or a bottom-up direction from small-to-large $^{25}$. Following a top-down direction, a whole-body is a complex organisation of eleven distinct organ systems including respiratory system, digestive system, nervous system, cardiovascular system, muscular system etc. The organ system is composed of several organs that work collaboratively to perform one or more specific functions, such as the brain in nervous system and the stomach in digestive system. Tissue links the cellular level and organ level, which is a group of cells with a common structure and function. For instance, the nervous tissue is composed of neurons and neuroglial cells for transmission of nerve impulse and protection, respectively. As shown in the scheme, the single epidermal cell and neuron are the smallest fundamental unit of structure and function in a living organism. Other than those cells forming tissues, there exist some other cells in the living organism, such as bacteria and fungus, which are single-celled prokaryotic and eukaryotic microorganisms. In the biological system, the molecules are also called biomolecules, and possess a wide range of sizes and structures with a vast variety of functions in cells, tissues and body-fluids. The most important biomolecules in a living organism are four kinds of macromolecules including nucleic acids, proteins, carbohydrates and lipids, which are the polymerisation products from their monomers and oligomers. Except for the macromolecules, a diverse range of small molecules also play a vital role in biological functions, such as metabolites (glucose, lactate), hormones, and neurotransmitters etc. All these molecules are formed 
from atoms (hydrogen, oxygen, carbon etc.) which are the smallest and most fundamental unit of matter.

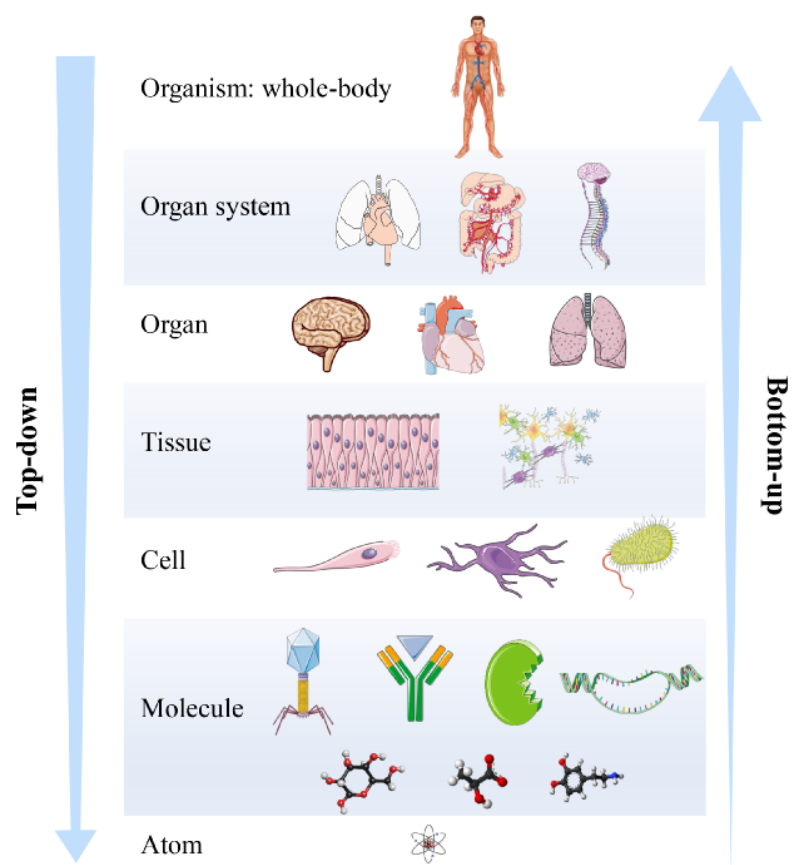

Figure 2.3 Scheme of hierarchical levels of a biological system.

The hierarchical levels of a biological system comprise of a large amount of information from the biological characteristics. The changes in biological quantities of the biological characteristics give us hints about health status and the encountered environments, because some biological phenomena fluctuate in a certain range, such as the body temperature of a human over the range of $35-42{ }^{\circ} \mathrm{C}$. Different levels of the biological system can be used for health monitoring for physical activities, healthy diet and exercise at the individual level. A better understanding of these biological characteristics and biomarkers can provide information for diagnosis of disease, screening and prevention of disease, as well as management of patients' responses to an exposure or a therapy during their treatment. It can also reflect the entire spectrum for categorised groups at a societal level. For instance, the establishment of gene mapping and specific DNA sequencing allow the stratification of genetic susceptibility to a specific "genotype" associated disease.

Table 2.1 summarises some common cases related to the application of physiological clues for health monitoring and disease diagnosis from different levels of a biological system. In some cases, the health monitoring for health status may be indicated 
immediately by a variety of physiological clues from macroscopic levels, including the general appearance by observing the breathing, facial features and expression, body motions from hand, leg, foot and waist, as well as vital signs from heart rate, blood pressure, temperature and dehydration rate ${ }^{26}$. For instance, a preliminary judgment can be made for the diagnosis of upper respiratory tract infection through coughing and wheezing in respiratory system. At the organ level, heartbeat rate and pulse rate can be used as an indicator for physical excise to analyse the intensity and duration of activities, and their disorder can be further used for fast clinical diagnosis of heart failure and cardiovascular disease. Despite the use of these indicators from macroscopic levels in empirical medicine, the accurate diagnosis of diseases requires the detection of biomarkers at microscopic levels.

Table 2.1. Employment of different levels of biological system for health monitoring and disease diagnosis.

\begin{tabular}{|c|c|c|c|}
\hline Biological level & Position & Indicator & $\begin{array}{l}\text { Health monitoring/disease } \\
\text { diagnosis }\end{array}$ \\
\hline Organism & Whole-body & Shuffling/festinating & $\begin{array}{l}\text { Classically in Parkinson's } \\
\text { disease }\end{array}$ \\
\hline Organ system & $\begin{array}{l}\text { Respiratory } \\
\text { system }\end{array}$ & Cough/wheeze & $\begin{array}{l}\text { Upper respiratory tract } \\
\text { infection }\end{array}$ \\
\hline \multirow{3}{*}{ Organ } & Skin & Temperature & Fever/infection \\
\hline & Heart/wrist & Beat/pulse rate & $\begin{array}{l}\text { Heart failure/cardiovascular } \\
\text { disease }\end{array}$ \\
\hline & Lung & Abnormal nodule & Lung cancer \\
\hline Tissue & Tissue & Abnormal cells & Tumor \\
\hline Cell & Biofluid & White blood cells & Infection \\
\hline \multirow{2}{*}{ Macromolecule } & \multirow{2}{*}{ Biofluid } & Nucleic acids & Pathogen infection \\
\hline & & Antigen & Infection \\
\hline Metabolite & Biofluid & Glucose & Routine diabetes monitoring \\
\hline
\end{tabular}

Biomarkers are defined as the biological characteristics that can be measured accurately and reproducibly as indicators of disease states, ranging from disease-specific molecules and cellular observation from biological media to physiological signal measurements ${ }^{27}$. The detection of multiple biomarkers provides a dynamic and powerful approach for a reliable and accurate evaluation of disease from the earliest stage to the terminal stage. For example, the diagnosis of lung cancer based on some certain symptoms usually involves a series of tests for biomarkers including imaging scan by X-ray and 
computerised tomography (CT), sputum cytology and tissue biopsy. At molecular level, a variety of molecules have been successfully used as biomarkers for health monitoring or disease diagnosis, ranging from macromolecules including enzymes, nucleic acids, antigen/antibody, collagen, virus, peptides and lipids, to small metabolite molecules such as glucose, lactate, creatinine, cholesterol, cortisol, vitamins, potassium and sodium cations. The knowledge gained from the biological system has driven the development and commercialisation of biosensors for self-monitoring such as the homebased devices for diabetes management based on the monitoring the blood glucose and paper-based sensing tools for pregnancy test based on the human chorionic gonadotropin (HCG) level.

\subsection{Organic Bioelectronics}

It is of particular significance to collect and analyse the biological phenomena in the manner of visually observable signal, which relates to the signal transfer or signal transduction into desired digital or analogue information. Such research has propelled the emergence and/or the development of a variety of disciplines and inter-disciplines, such as systems biology, molecular biology, cell biology, neurology, genetics, radiology, sensors, material science, laboratory instrument technology. Originally inspired by the pioneering study of bioelectricity from frog tissues by Galvani, efforts have been devoted to the development of electronic devices for living organs and tissues. A good example is the neural interface designed for bridging between analogue nervous systems and electronic devices consisting of three key modules, e.g. tissue interface, sensing interface and neural signal processing unit. Another example is the application of glucose biosensors for detection of blood glucose level by using glucose oxidase (GOx) as the biorecognition element for specific recognition and subsequent transduction of intermediate into electrons collected by a conductive electrode. One of the key challenges in signal transfer/transduction is the establishment of an efficient biotic/abiotic interface.

Rapid advances have been made in recent years in the development of interfacing materials to bridge the gap at the biotic/abiotic interface, including a large variety of inorganic materials such as metals, silicon and ceramics, organic materials such as biomacromolecules (e.g. peptides), biomimetic molecules inspired from nature (e.g. chiral molecules), hydrogels and CPs, as well as the rational design of the structure, morphology and post-functionalisation ${ }^{28-30}$. The employment of inorganic materials for biointerfaces encountered several limitations on the two side of the interface (inorganic/bioorganic) due to their incompatibilities in terms of chemical structure (inorganic $v s$ organic), different mechanical properties (high vs low Young's modulus) 
and different electrical properties (electronic $v s$ ionic conduction) ${ }^{6}$. Organic materials, especially CPs, have been proposed as an alternative material to overcome these issues for the fabrication of organic electronic devices used for bioelectronics, leading to the new terminology of "Organic bioelectronics (OBEs)" in the last two decades.

OBEs are dedicated to research into the properties and mechanisms of organic electronic materials, and the development and studies of organic electronic devices, which are usually composed of CPs as the active elements in combination with other functional materials $^{31}$. CPs are emerging as promising interfacing materials in OBEs because they share sufficient similarities in their organic chemical nature with biological systems. On the other hand, their rich synthetic chemistry endows CPs with versatile functional groups for biomolecule coupling with increased compatibility bridging between the organic electronics world and bioelectronics. Moreover, the soft characteristic of CPs enable their integration for the development of flexible organic electronic devices with low-cost and light-weight, compared to conventional rigid materials, such as metals and semiconductors. More details regarding to CPs are given in Chapter 4. Due to these unique properties of $\mathrm{CPs}, \mathrm{CP}$-based organic electronic devices have been applied widely in the biomedical field for bioelectronics including neural interfaces for signal recording and stimulation, electronic surfaces and scaffolds for cell and tissue culture, artificial electronic skin with flexibility and display features, chemical and drug delivery system for treatment and stimulation, organic field-effect transistor (OFET) based sensors and biosensors, actuators and so on (Figure 2.4).

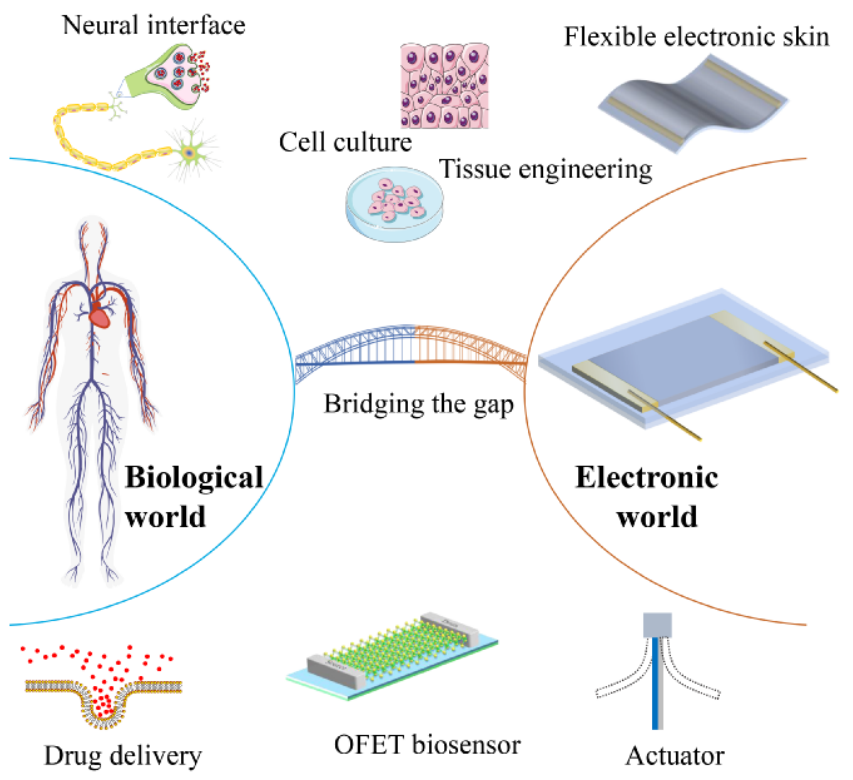

Figure 2.4 Applications of OBEs bridging the biological world and electronic world. 
Chapter 2 


\section{Chapter 3 Sensors and Biosensors in Bioelectronics}

Conventional analytical methods and techniques employ laboratory and bench-top instruments have been intensively and widely applied in acquiring bioinformation from the aforementioned hierarchical levels of a biological system for disease diagnosis. Such instruments provide a comprehensive and accurate evaluation in hospital or laboratory level, such as automatic biochemistry analysers for multi-characteristic analysis in different biofluids including, but not limited to blood, serum, plasma, urine and cerebrospinal fluid, and optical bioimaging and spectroscopy system for a certain of pathological sites including CT scan, MRI, optical coherence tomography (OCT), ultrasound, infrared (IR), X-ray and Raman. However, they also have some disadvantages in expansion of the application scope in decentralised locations in a far more cost-effective manner, because of the expensive materials and instruments, timeconsuming and needing of trained personnel for operation. Taking advantage of their fast response, low-cost, portability and easy-of-operation, sensors and biosensors proved invaluable for quantitative and accurate determination of characteristics in the form of PoC devices in recent decades, as well as their potential for the development of wearable sensing and biosensing devices for real-time and remote health monitoring. This chapter focuses on the development of sensors and biosensors in bioelectronic applications. Sensors and biosensors are discussed separately from the recognition and signal transduction perspective.

\subsection{Sensors}

\subsubsection{Sensor Definition}

A sensor is a device for event detection and signal transduction to provide a certain type of response directly related to the quantity of a specific change. In the range of bioelectronics related to a living organism, a sensor can convert the physical (e.g. body motions, temperature) or physiological parameters (e.g., uric acid, glucose) into a signal which can be measured optically or electrically. Figure 3.1 illustrates the main components of a sensor including a sensing element, a transduction element and a signal processing element. In a sensor system, the physical, chemical or biological change of the system to be determined is specifically recognised by the sensing elements, followed by the transduction of the input change into a readable signal in quantity in a variety of techniques, such as thermometric, electrochemical, optical, piezoelectric, magnetic and micromechanical methods. The signal is received by a signal processing element, which 
may undergo a series of extra process with amplification and filtration, analysis, transmission and feedback.

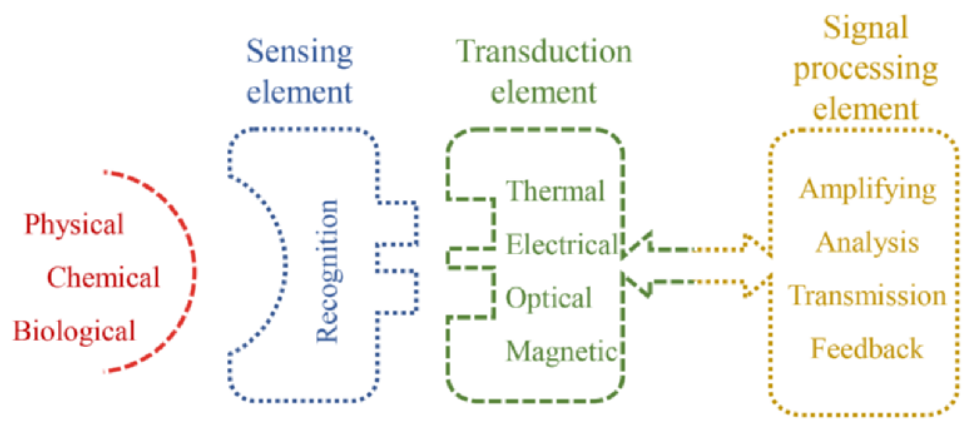

Figure 3.1 Main components of a sensor system.

\subsubsection{Sensor Criteria}

There are several features can be used for the evaluation of a sensor for its static and dynamic performance, such as accuracy, sensitivity, selectivity, limit of detection (LoD), dynamic range, response time, stability and repeatability. The accuracy of a sensor is the difference or error of the signal between the actual value and the detected value collected from the sensing and transduction elements. For instance, $+/-15 \%$ accuracy has been recommended for the home blood monitoring systems by the U.S. Food and Drug Administration (FDA). Some of the other criteria are illustrated in Figure 3.2.

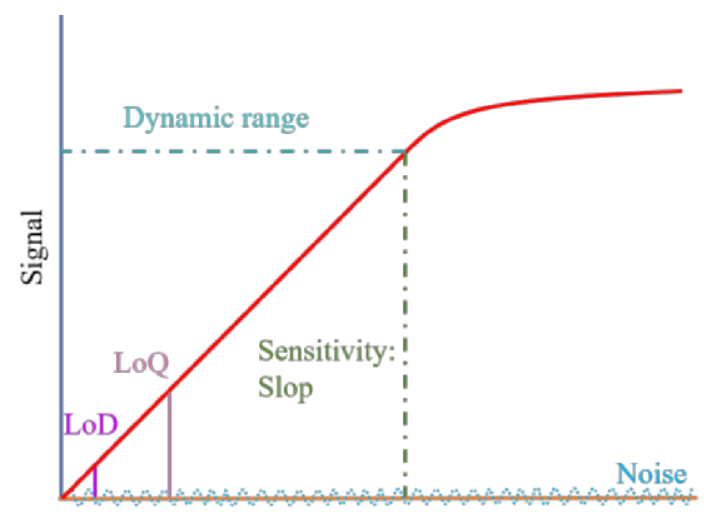

Physi/chemi/bio Changes

Figure 3.2 Criteria usually used to assess a sensor.

Dynamic range is the working range of the physical, chemical or biological changes from minimum to maximum that a certain response value can be assumed into the 
corresponding change in quantity. The slope of the dynamic range to the function of change is usually used to define the sensitivity, indicating the response value per change. Noise is the unwanted wave vibration caused by the external circumstance or internal structure, which determines the resolution and limits the ultimate performance that can be obtained by a given sensor in terms of signal to noise ratio.

In chemical sensors and biosensors, LoD and limit of quantification (LoQ) are two important performance characteristics describing the lowest level of the changes that can be distinguished from the blank without necessary quantification and can be determined with acceptable accuracy, respectively, by taking consideration of the blank and signal vibration. There are different methods for the estimation of LoD and LoQ. For a linear regression, the LoD and LoQ are expressed as the following equations,

$$
\begin{aligned}
& \mathrm{LoD}=3 S_{a} / b, \\
& \mathrm{LoQ}=10 S_{a} / b,
\end{aligned}
$$

where $S_{a}$ is the standard deviation of the signal response by either y-residuals, or yintercepts of the regression lines by 10 times, and $b$ is the slope of the calibration curve according to the International Union of Pure and Applied Chemistry (IUPAC) ${ }^{32}$. Alternatively, the signal-to-noise $(\mathrm{S} / \mathrm{N})$ method is used by calculating the noise magnitude, in which 3 and 10 are generally accepted as the $\mathrm{S} / \mathrm{N}$ ratio to estimate the LoD and $\mathrm{LoQ}^{32}$.

Response time is defined as the time required by the sensor system to respond to a loading of change from its initial state, and $90 \%$ of the final response value is set to specify the response time. Selectivity or specificity is the ability of a sensor system to determine the level of a specific substance in the presence of other interferences, which is also called anti-inference ability. Stability is another criterion to assess a sensor system including the long-term running stability and storage stability. Whether it is repeatable for a measurement by several times or by several batches is defined as the repeatability and reproducibility, respectively, which are closely related to the precision by the relative standard deviation (RSD) value in statistics.

\subsubsection{Sensor Classification}

Sensors are classified based upon several different perspectives. First, sensors can be divided into two categories of active and passive sensors, in which active sensors need an external excitation signal to generate the output signal, while passive sensors do not require any external signal. According to the datatype, sensors are classified into analogue sensors and digital sensors. Analogue sensors can produce a continuous output signal that is generally proportional to the quantity being measured, such as a thermometer responding to temperature changes continuously. Digital sensors produce 
a discrete digital output signal, i.e. non-continuous value. Another type of classification is based on the application for various targets, such as mechanical sensors for velocity, acceleration, position, flow and deformation sensing and control, physical sensors for humidity, pressure, strain and temperature detection, chemical sensors for gaseous, liquid and solid molecules concentration detection, and biosensors for biomarkers and other biological characteristics detection. What's more, the most commonly used classification for sensors in the academic community relies on the transduction methods for phenomenon conversion into readable signal including electrochemical, photoelectric, thermoelectric, magnetic, piezoelectric, optical methods and so on.

\subsection{Electrochemical Sensors}

Electrochemical sensors are a subclass of sensors with an electrode as the transducer element that converts various physical, chemical and biological parameters into an electrical signal in quantity. The field of electrochemical sensors is attractive and expanding very fast because of their capability to deliver fast, precise, sensitive, selective analysis with an easy-to-use device. The range of applications of the electrochemical sensors includes, but not limited to, food monitoring, environmental and agricultural monitoring, medical and healthcare monitoring. Recent development of material science and nanotechnology enables the improvement of electrochemical performance via the functionalisation of the electrode surface with advanced materials. In addition. the potential for miniaturisation and fabrication of portable devices can be used for the exploration of the next generation of flexible and wearable sensing devices. This section summarises the commonly used electrochemical techniques in electrochemical sensors, chemically modified electrodes to improve the electrochemical performance with advanced materials compared to conventional bare electrodes, and the applications of electrochemical sensors for healthcare monitoring.

\subsubsection{Electrochemical Techniques}

The recognition and conversion of physical, chemical and biological parameters into electrical signals are carried out sequentially at the electrode interface by the sensing element and transducer by various electrochemical techniques. Such signal transduction can be quantified according to the changes of current, potential, resistance, capacitance, charge transfer resistance at the electrode surface of a transducer by different electrochemical techniques consisting of voltammetry, amperometry, potentiometry, conductometry, coulometry and impedance. Table 3.1 summarises these electrochemical techniques, physicochemical properties obtained and their applications for healthcare monitoring. Some of these key techniques used in this thesis are explained in detail below. 
Table 3.1 Summarisation of the selected electrochemical techniques.

\begin{tabular}{lll}
\hline Technique & $\begin{array}{l}\text { Physicochemical } \\
\text { properties }\end{array}$ & Applications \\
\hline Voltammetry & Potential-current & Analyte redox peaks, reversibility, electrode kinetics \\
Amperometry & Current-time & Deposition, chemical-/bio- sensing \\
Impedance & Impedance, diffusion & Electrode kinetics \\
Potentiometry & Potential difference & Ion-selective electrode (e.g. pH meter) \\
Conductometry & Resistance & Titration analysis \\
Coulometry & Current-time, charge & Titration analysis, deposition, chemical-/bio- sensing \\
\hline
\end{tabular}

As shown in Figure 3.3, an electrochemical cell is usually connected with a potentiostat for most of the electrochemical techniques and equipped with a three-electrode system including a working electrode, a reference electrode and a counter electrode, in which the working electrode and reference electrode form a closed circuit to measure and control the applied potential precisely, and the working electrode and counter electrode form a closed circuit to pass and measure the current. The criteria for reference electrodes are the capability to provide a well-defined and stable equilibrium potential. Silver/silver chloride $(\mathrm{Ag} / \mathrm{AgCl})$ and silver/silver ion $\left(\mathrm{Ag} / \mathrm{Ag}^{+}\right)$electrodes were used for aqueous solution and non-aqueous solution, respectively, in this thesis. Inert materials are used to be chosen as the counter electrode to minimise the side reactions, and the surface area of the counter electrode should be much larger than that of the working electrode, such as platinum (Pt) wire or plate ${ }^{33}$.

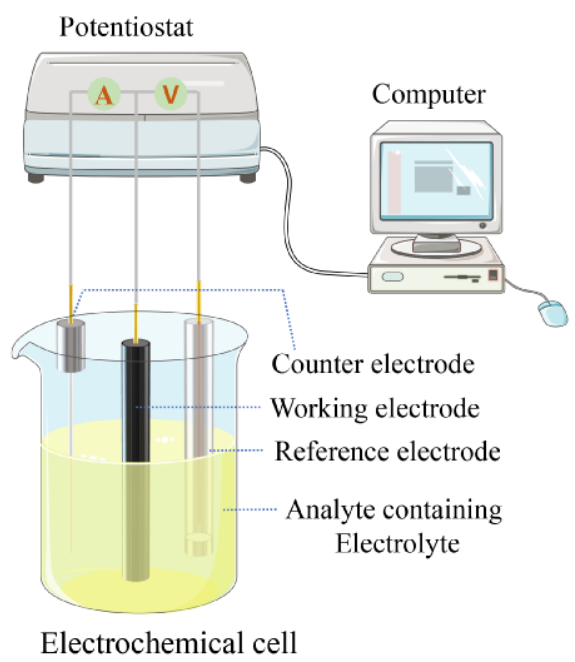

Figure 3.3 Electrochemical cell with a three-electrode system. 
Voltammetry is one of the most powerful electrochemical techniques to study the halfcell reactivity of an analyte. In a voltammetric experiment, dynamic potentials are applied to the three-electrode system and the current response is measured. The applied potentials relative to a reference electrode are dynamic and change as a function of the time. According to the relationship between the dynamic potential and the time, voltammetric methods can be classified into cyclic voltammetry (CV), linear sweep voltammetry (LSV), differential pulse voltammetry (DPV), square wave voltammetry (SWV), anodic/cathodic stripping voltammetry (ASV/CSV) etc. Figure 3.4 illustrates the $\mathrm{CV}$ and DPV techniques used in this thesis. In CV, the potential sweeps between the setting V1 and V2 (potential window) at a constant rate (scan rate), and an oxidation peak and a reduction peak originating from the redox reactions of the molecules in the electrolyte can be observed in the potential-current curve in the presence of a pair of reversible redox probe, such as ferricyanide/ferrocyanide $\left(\mathrm{Fe}^{\mathrm{III}}(\mathrm{CN})_{6}{ }^{3-} / \mathrm{Fe}^{\mathrm{II}}(\mathrm{CN})_{6}{ }^{4-}\right)$ in the following reaction.

$$
\mathrm{Fe}^{\mathrm{III}}(\mathrm{CN}) 6^{3-}+\mathrm{e}^{-} \rightleftarrows \mathrm{Fe}^{\mathrm{II}}(\mathrm{CN})_{6}^{4-}
$$

(a)

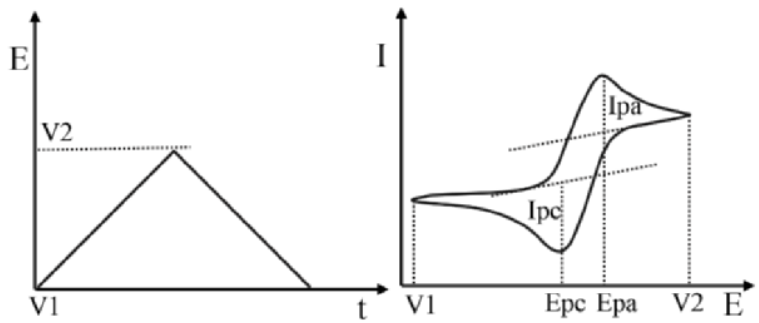

(b)

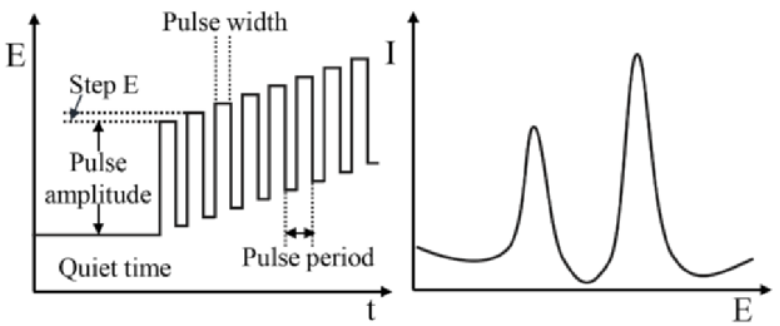

Figure 3.4 CV (a) and DPV (b) techniques with the potential-time curve (left) and potential-current curve (right).

Various information can be obtained from $\mathrm{CV}$, including quantitative level, redox potential, diffusion coefficient, electrochemical reaction rate for the analytes in the electrolyte, as well as electrode kinetics, electron transfer and charge transport at a modified electrode surface. In Paper I-V, CV was employed to investigate the oxidation or reduction potential for $\mathrm{NADH}$, dopamine, ascorbic acid, uric acid, ferricyanide and 
hydrogen peroxide at the modified electrode surface. Besides, CV was also used to evaluate the properties of the modified electrode comprising of the surface charge in Paper I, II and IV, active surface area in Paper I, interfacial capacitance in Paper III, conductivity related electron transfer rate in Paper $\mathbf{V}$. DPV is a derivative technique of LSV and staircase voltammetry, involving an applied pulse with a constant amplitude for the increase of the potential as a function of time. Since the potential of each subsequent pulse is slightly higher than the previous one, the rate of decay of the charging current and the faradic current are different, in which the charging current decays exponentially while the faradic current decays as a function of $1 /(\text { time })^{1 / 2}$. Therefore, the charging current is negligible and thus DPV possesses higher sensitivity than other voltammetric techniques. In Paper II, DPV was applied to measure the concentration of dopamine at a low concentration level.

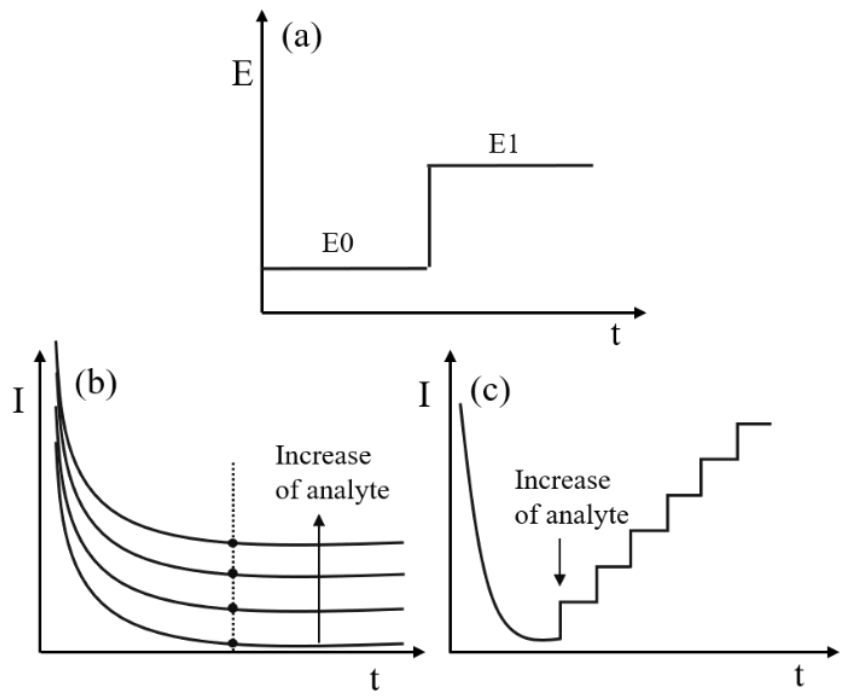

Figure 3.5 (a) Chronoamperometry or amperometry with the current-time curve, (b) current-time curve at different concentration of analyte in static (i.e. non-stirred) system, (c) current-time curve with successive addition of analyte into the solution in a stirred system.

Unlike the dynamic potential in voltammetry, chronoamperometry or amperometry are potentiostatic techniques. The current flow at the working electrode is measured as a function of time with a constant applied potential (Figure 3.5). And the current collected from static (i.e. non-stirred) system is correlated with the concentration of the analytes diffused to the working electrode interface from the bulk solution according to the Cottrell equation ${ }^{34}$ : 


$$
\mathrm{I}=\mathrm{n} \mathrm{F} \mathrm{C} \mathrm{A} \mathrm{D}{ }^{1 / 2} \pi^{-1 / 2} \mathrm{t}^{-1 / 2}
$$

where $\mathrm{I}$ is the current, $\mathrm{n}$ is the number of electrons, $\mathrm{F}$ is the Faraday's constant, $\mathrm{C}$ is the analyte concentration, $\mathrm{D}$ is the diffusion coefficient, $\mathrm{t}$ is the time. In Paper $\mathbf{V}$, amperometry with a non-stirred system was applied to get a calibration curve for lactate in artificial sweat with a wide linear range. On the other hand, in a stirred system, the successive injections of the analyte into the electrolyte caused a stepwise increase in the current response, and this was employed in Paper I, IV and V.

Electrochemical impedance spectroscopy (EIS) is a very sensitive and powerful technique to evaluate the modification of the electrode interface, molecule diffusion kinetics and interactions with the electrode surface. The obtained impedance spectrum resulting from the AC signal over a wide range of frequencies (usually $100 \mathrm{kHz}$ to 0.01 $\mathrm{Hz}$ ) can be modelled with a suitable circuit, i.e. equivalent circuit. A commonly used equivalent circuit is Randles model circuit (Figure 3.6) that are composed of the mixed kinetic and charge-transfer control, in which Rs is the solution resistance, Rct is the charge-transfer resistance, CPE is the constant phase element for a rough interface $(\mathrm{n}=$ 0 is a pure resistor, $\mathrm{n}=1$ is a pure capacitor), and $\mathrm{W}$ is the Warburg impedance by the diffusion contribution. EIS was employed to evaluate the electrode kinetics for selective discrimination of dopamine in Paper II, and was used to calculate the equivalent series resistance (ESR) and Rct of the modified electrode in Paper III.

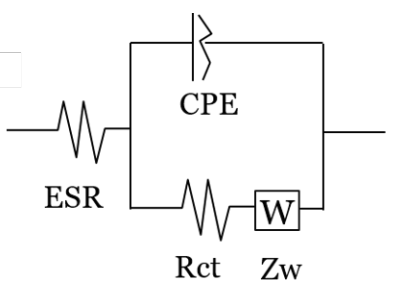

Figure 3.6 Randles model circuit.

\subsubsection{Chemically Modified Electrodes}

Chemically modified electrodes (CMEs) have been widely researched for applications ranging from sensing and biosensing to energy storage including capacitors and batteries $^{35}$. Especially in electrochemical sensing applications, almost all of the electrochemical reaction processes occur at the electrode interface. The conventional working electrodes used extensively are carbon-based solid electrodes including glassy carbon electrode (GCE), pyrolytic graphite electrodes, boron doped diamond electrode and other types of carbon electrode (e.g. laser induced graphene electrode prepared in Paper $\mathbf{V}$ in this thesis), and screen printed electrodes using carbon or metal ink, noble metal electrodes involving $\mathrm{Pt}, \mathrm{Au}$ and $\mathrm{Pd}$. Such bare electrodes usually surfer from the 
limitation of sluggish electrode kinetics for a large variety of analytes. Therefore, the modification or functionalisation of the electrode surface is of crucial importance in electrochemical reactions in sensing. There are several advantages of CMEs compared to the conventional bare working electrodes for electrochemical sensing including:

1) enhanced charge transfer kinetics with low overpotential, especially when the electrochemical process is either not possible or difficult to achieve for poorly active analytes;

2) improved sensitivity via increased surface area with accessible reactive sites and surface activity;

3) improved selectivity for the analytes in the presence of interferences due to the recognition effect;

4) enhanced chemical and electrochemical stability and reduced fouling ${ }^{36}$.

Considerable effort has therefore been devoted to developing CMEs using advanced materials with desirable properties that can boost the electrochemical sensing signal transduction. The successful modification or functionalisation of the electrodes can be achieved in a variety of physical and chemical methods, which can be categorised into physical deposition, electrochemical deposition/polymerisation and chemical bonding as shown in Figure 3.7. Physical deposition of the electrode surface is the use of either physical techniques (e.g. UV and laser) for treatment of the surface, or the direct coating of active material composites using spin-coating, dip-coating, drop-casting and bladecoating in the formation of suspension, ink and paste of carbon materials (e.g. CNTs, graphene, graphite), metallic materials (e.g. metal particles, Prussian blue) and polymers (e.g. polyelectrolytes, redox polymers and CPs). For instance, water processable PEDOT colloidal microparticles (PEDOT CMs) suspension was deposited (Figure 3.7a) on the bare GCE surface in Paper $\mathbf{I}$, resulting in a hierarchically-structured electrode surface with increased sensitivity than the dense film of benchmark poly $(3,4-$ ethylenedioxythiophene)-poly(styrenesulfonate) (PEDOT:PSS).

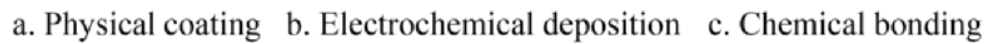
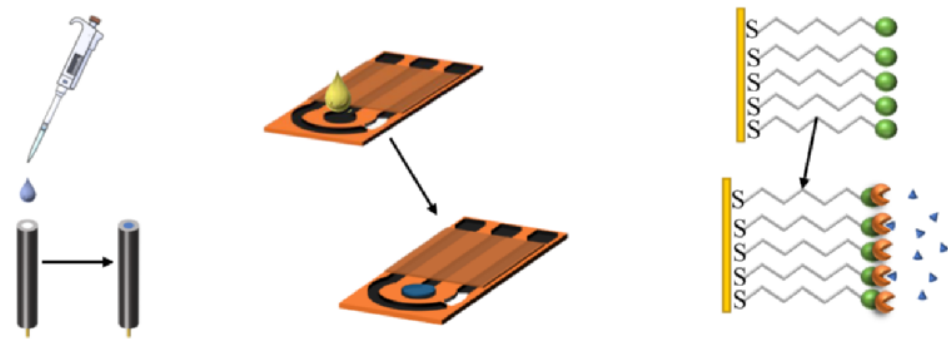

Figure 3.7 Three examples for chemically modified electrode using physical coating (a), electrochemical deposition (b) and chemical bonding via gold-thiols (c). 
Besides physical deposition, electrochemical deposition/polymerisation (Figure 3.7b) is another convenient technique for functionalisation of the electrode surface using the electrochemical techniques mentioned above. Inorganic materials including metal nanoparticles, metal oxide, metal complex and carbon are a large category of the materials that can be electrochemically deposited on the electrode surface. In Pager V, Prussian blue $\left(\mathrm{KFe}^{\mathrm{II}}\left[\mathrm{Fe}^{\mathrm{II}}(\mathrm{CN})_{6}\right]\right)$ was electrochemically deposited on the working electrode via chronoamperometry at a constant potential using $\mathrm{Fe}(\mathrm{CN})_{6}{ }^{3-}$ and $\mathrm{FeCl}_{3}$ as precursors following the reactions listed below ${ }^{37,38}$ :

$$
\begin{gathered}
\mathrm{Fe}^{3+}+\mathrm{e}^{-} \rightarrow \mathrm{Fe}^{2+} \quad \text { at about } 0.4 \mathrm{~V} \\
\mathrm{Fe}^{2+}+\mathrm{Fe}(\mathrm{CN})_{6}^{3-}+\mathrm{K}^{+} \rightarrow \mathrm{KFe}^{\mathrm{III}}\left[\mathrm{Fe}^{\mathrm{II}}(\mathrm{CN})_{6}\right] \\
\mathrm{KFe}^{\mathrm{III}}\left[\mathrm{Fe}^{\mathrm{II}}(\mathrm{CN})_{6}\right]+\mathrm{e}^{-}+\mathrm{K}^{+} \rightleftarrows \mathrm{K}_{2} \mathrm{Fe}^{\mathrm{II}}\left[\mathrm{Fe}^{\mathrm{II}}(\mathrm{CN})_{6}\right] \\
\mathrm{KFe}^{\mathrm{II}}\left[\mathrm{Fe}^{\mathrm{II}}(\mathrm{CN})_{6}\right]+\mathrm{H}_{2} \mathrm{O}_{2} \rightarrow \mathrm{KFe}^{\mathrm{III}}\left[\mathrm{Fe}^{\mathrm{II}}(\mathrm{CN})_{6}\right]+\mathrm{H}_{2} \mathrm{O}
\end{gathered}
$$

As the metal complex, the reduced form of Prussian blue, i.e. Prussian white $\left(\mathrm{K}_{2} \mathrm{Fe}^{\mathrm{II}}\left[\mathrm{Fe}^{\mathrm{II}}(\mathrm{CN})_{6}\right]\right)$, possesses excellent reduction catalytic activity towards hydrogen peroxide $\left(\mathrm{H}_{2} \mathrm{O}_{2}\right)$, which is widely accepted as an "artificial peroxidase".

The electrochemical deposition of these functional materials as mediators, catalysts or morphology-templates endows the electrode surface with enhanced catalytic activity, active surface area and conductivity compared to that of a bare electrode, and can be used for the fabrication of an electrochemical sensor with high sensitivity. Using similar electrochemical techniques, different forms of polymers including polyelectrolytes, redox polymers and CPs can be electropolymerised onto the electrode surface to form good films. The electrochemical polymerisation of CPs used in Paper II-V is discussed in Chapter 4 in detail. In addition to CPs, some redox polymers can be electropolymerised on the electrode surface acting as heterogeneous redox mediators for electrochemical sensing of analytes, such as poly(malachite green $)^{39}$ and poly(1naphthylamine $)^{40}$ etc.

The introduction of functional groups on the electrode surface can tune its surface charge, wettability and active functional groups as anchoring sites for post-treatment with other functional molecules or materials. For instance, the covalent bond between gold and sulphur contributes to a robust and modifiable gold electrode surface (RS-Au), especially for the self-assembled monolayers of thiols on planar gold surfaces (Figure 3.7 c). The attached functional groups from thiols or post-anchored recognition element can participate directly in the interactions with analyte molecules in the solution based on adsorption, hydrogen or coordination bonds and redox reactions, which may influence the charge transfer resistance or capacitance at the electrode interface and are thus used for electrochemical sensing via impedimetric or capacitive techniques ${ }^{41,42}$. A 
similar mechanism is also applied for the introduction of azide groups, amine groups and carboxylic acid onto the electrode surface and their post-treatment via "clickchemistry" ${ }^{\prime 3}$.

\subsubsection{Electrochemical Sensors for Healthcare Monitoring}

Owing to the advantages of fast response, high sensitivity, easy-of-operation, as well as the capability for miniaturisation and fabrication into portable and even wearable devices, electrochemical sensors are one of the most promising candidates for PoC devices intended for healthcare monitoring. Such healthcare monitoring relies on the detection of a variety of physiological parameters, including physical phenomena, chemical sensing and biosensing, in which biosensing is discussed in section 3.3 in detail.

The applications of electrochemical sensors in chemical sensing refer to the detection of specific chemical elements, such as ions, molecules and compounds along with their concentration level, serving as indicators for healthcare management or intermediate signal transduction for biosensing of other metabolites.

Nicotinamide adenine dinucleotide (NADH) is the most important cofactor found in human body since it participates in reactions with more than 300 dehydrogenases associated with $\mathrm{NAD}^{+}$(the oxidised form). Deficiency of NADH will cause the energy deficit owing to the lack of ATP production at the cellular level, while excessive levels of NADH will lead to insomnia, anxiety, fatigue and overstimulation ${ }^{44}$. Electrochemical oxidation of $\mathrm{NADH}$ at an electrode surface has been proven to be an efficient way for the determination of NADH level. As shown in Figure 3.8, the mechanism of the electrochemical oxidation of NADH undergoes a two-electron and one-proton process following an "electron transfer-chemical reaction-electron transfer (ECE)" mechanism. However, the electrochemical oxidation of NADH at conventional electrode surface usually requires a high overpotential, such as $0.735 \mathrm{~V}$ at bare GCE based on our results in Paper I, which will result in potential interferences from other molecules and electrode fouling due to the adsorption of intermediates. To diminish these problems caused by the high overpotential, functionalised electrodes have been widely developed to reduce the overpotential and increase reaction kinetics with the assistance of redox mediators, carbon-based nanomaterials, metallic nanomaterials and $\mathrm{CPs}^{45}$. This thesis demonstrates two strategies to increase the electrochemical oxidation reaction kinetics of NADH with decreased peak potential and increased sensitivity. In Paper I, we demonstrated the facile preparation of a positively-charged PEDOT interface for the electrooxidation of $\mathrm{NADH}$, in which the intrinsic positive charge of the PEDOT microparticles facilitated the detection of negatively-charged NADH molecules by electrostatic accumulation. In the other case, a three-dimensional PEDOT nanofibrous 
network with its high specific surface area resulted in a low charge transfer resistance and high transduction activity towards NADH as described in Paper IV.
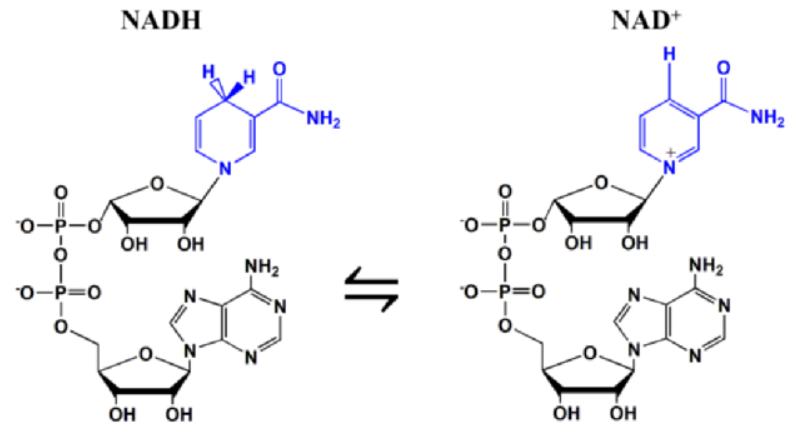

$$
\begin{aligned}
& \text { Electron transfer step: } \mathrm{NADH} \rightarrow \mathrm{NADH} \bullet^{+} \mathrm{e}^{-} \\
& \text {Chemical reaction step: NADH } \bullet^{+} \rightarrow \mathrm{NAD} \bullet+\mathrm{H}^{+} \\
& \text {Electron transfer step: NAD } \bullet \rightarrow \mathrm{NAD}^{+}+\mathrm{e}^{-}
\end{aligned}
$$

Figure 3.8 Structure of $\mathrm{NADH}$ and $\mathrm{NAD}^{+}$and the electrochemical oxidation process form $\mathrm{NADH}$ to $\mathrm{NAD}^{+}$.

Hydrogen peroxide $\left(\mathrm{H}_{2} \mathrm{O}_{2}\right)$ as a kind of reactive oxygen species, is another important biomarker that has attracted extensive research interest for its fast and accurate detection, because the expression level of $\mathrm{H}_{2} \mathrm{O}_{2}$ in biological tissues or fluids are of great significance, while the long-term excessive and abnormal expression of $\mathrm{H}_{2} \mathrm{O}_{2}$ will impact the intracellular signaling pathways of healthy cells and the cellular constituents, causing some neurodegenerative diseases and cancers ${ }^{46}$. The detection of $\mathrm{H}_{2} \mathrm{O}_{2}$ can also be linked with many enzymatic catalytic reactions for a lot of metabolites, such as glucose based on GOx and lactate based on lactate oxidase (LOx) ${ }^{47}$. Horseradish peroxidase (HRP) had been widely used for enzymatic catalytic detection of $\mathrm{H}_{2} \mathrm{O}_{2}$ with high selectivity, but the performance of the enzymatic electrochemical sensors are still limited owing to the unsatisfactory stability derived from the intrinsic nature of the enzyme. Therefore, much effort has been devoted to the development of enzyme-free electrocatalysts for $\mathrm{H}_{2} \mathrm{O}_{2}$ sensing with several classifications of active materials involving carbon-based nanomaterials (e.g. CNTs, graphene, carbon quantum dots), noble metal nanoparticles (e.g. Pt, $\mathrm{Pd}$ ), metal oxides (e.g. $\left.\mathrm{Co}_{3} \mathrm{O}_{4}, \mathrm{MnO}_{2}\right)$ and dyes (e.g. Prussian blue, toluidine blue $)^{48} \cdot \mathrm{H}_{2} \mathrm{O}_{2}$ can be either electrochemical oxidised via a twoelectron and two-proton process at Pt electrode surface ${ }^{49}$ or reduced by Prussian blue via a two-electron process as shown in Figure 3.9. In Paper III, PEDOT doped with sulphonate-coupled reduced graphene oxide (PEDOT:S-RGO) was evaluated as electrochemical transduction for $\mathrm{H}_{2} \mathrm{O}_{2}$ and showed decreased onset potential and 
increased oxidation current compared to that of PEDOT:GO and PEDOT:RGO. In Paper V, Prussian blue was electrodeposited on 3D porous electrode surface as the catalyst towards the enzymatic intermediate $\mathrm{H}_{2} \mathrm{O}_{2}$ from LOx.

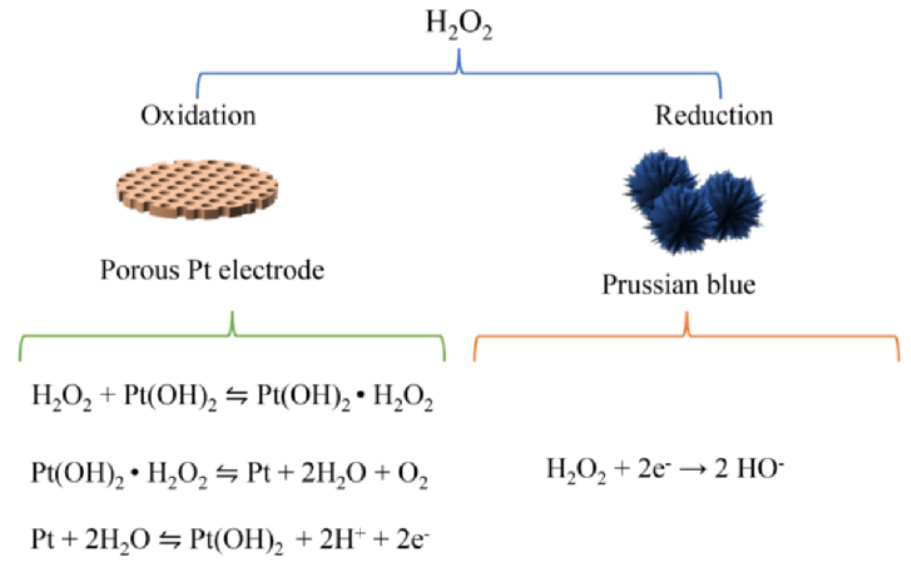

Figure 3.9 Electrochemical oxidation and reduction mechanism of $\mathrm{H}_{2} \mathrm{O}_{2}$ by porous $\mathrm{Pt}$ electrode and Prussian blue, respectively.

As one of the crucial neurotransmitters in cardiovascular, renal, hormonal, and central nervous systems, the physiological level of dopamine (DA) in extracellular fluid is vitally important in the clinical diagnosis of the DA-correlated neurological disorders, such as Parkinson's and Alzheimer's diseases. Electrochemical technique is one of the promising methods for the DA determination based on its electrochemical oxidation via two-electron and two proton transfer process as illustrated in Figure 3.10. However, it is challenging to discriminate DA from the co-existing ascorbic acid (AA) and uric acid (UA) in physiological samples because of their closely similar oxidation potentials. In order to diminish this problem, many functionalised electrodes have been constructed to separate the oxidation peaks of DA, AA and UA based on the carbon nanomaterials, metal complexes, polymers, redox mediators or their composites. On the other hand, the different protonated/deprotonated forms of AA $(\mathrm{pKa}=4.2), \mathrm{UA}(\mathrm{pKa}=5.4)$ with DA $(\mathrm{pKa}=8.9)$ under physiological conditions $(\mathrm{pH} 7.4)$ provides a charge effect to circumvent this issue by attracting the cationic DA to a negatively charged electrode interface while repelling the anionic AA and UA. In Paper II, negatively charged carboxylic-acid-group-functionalised PEDOT (PEDOT:COOH) showed improved electrode kinetics and facilitated the detection and discrimination of DA from the major interferents AA and UA. 


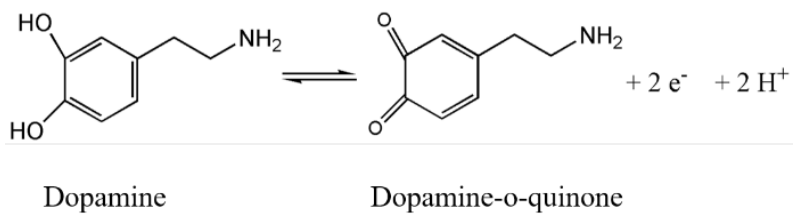

Figure 3.10 Dopamine structure and its electrochemical oxidation.

Apart from the detection of these chemical biomarkers by electrochemical sensors, a variety of physical phenomena involving temperature and humidity of the human body, strain and pressure from touch, voice, muscle movement for body motions, heartbeat rate and jugular venous pulse rate have been used as the indicators for human-activity monitoring and personal healthcare by electrochemical sensors ${ }^{50}$.

\subsection{Biosensors}

The monitoring of physical phenomena and chemical changes in the living systems described above does not go far enough to fulfill the demanding for disease diagnosis and daily healthcare management, which is supplemented by the utmost important bioinformation from biological or biochemical processes/levels. However, the conversion of such bioinformation into electrical signal has been challenging since the complexity of bridging the biological world to electronics. Biosensors discussed in this section are an attractive strategy.

\subsubsection{Biosensor Principle}

A biosensor, as classically defined, is a specific type of chemical sensor incorporating biological or biologically-derived receptors as sensing elements to harness the exquisite specificity and sensitivity of living systems in conjunction with electronic transducers and processors, to either provide data directly or to actuate an appropriate response. The schematic diagram in Figure 3.11 illustrates the components of a biosensor. The analytes from the living systems consist of the biomarkers discussed in section 2.2 in different levels, such as a large variety of metabolites, nucleic acids, antigen/antibody, microorganism, viruses and cells, which can provide significant information for their presence/absence, and activity and function of the human body. The recognitions of these analytes are realised by bioreceptors with the specific interactions or reactions resulted from the biological events. Subsequently, the transducers convert these events into a quantifiable and readable signal, which are then analysed and displayed as the electrical signal. 


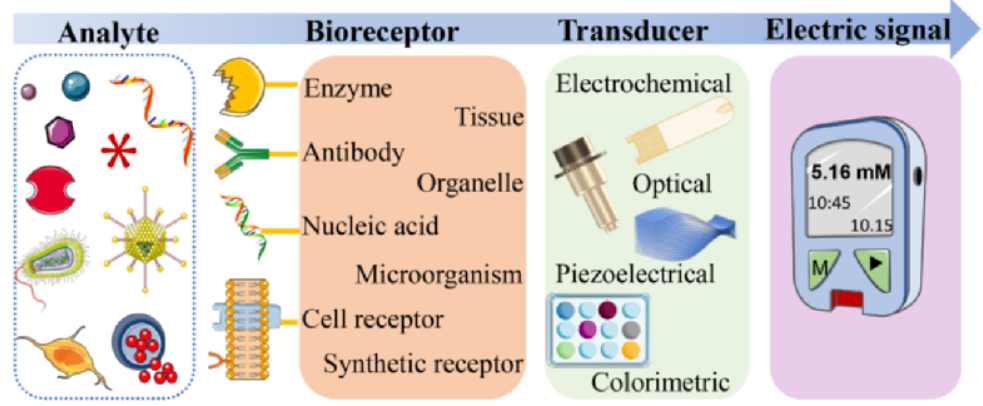

Figure 3.11 Components of a biosensor.

\subsubsection{Biosensor Classification}

Bioreceptors originating from natural systems such as enzyme, antibody, nucleic acid, cell receptor, tissue, organelle and microorganism can be used for the fabrication of biosensors. However, some of these natural bioreceptors may have several intrinsic limitations in their application, such as high cost, low stability and complicated methodology, and in some cases, no suitable bioreceptors are existing in nature. To circumvent these intrinsic limitations, synthetic receptors can offer an alternative and promising approach by mimicking the natural biorecognition events and biological entities, such as molecularly imprinted polymers ${ }^{51}$ and aptamers ${ }^{52}$.

Biosensors can be classified into (i) catalytic biosensors, typical examples are enzymatic biosensors; and (ii) affinity biosensors, typical examples are immunosensors and nucleic acid biosensors, based on the difference in the biorecognition principle. Catalytic biosensors employ catalysts as bioreceptors including enzymes, biomimetic/synthetic catalysts, cells and microorganisms etc., in which the sensing principle relies on the detection of biocatalytic reactions in the presence of the target analyte. In contrast, affinity biosensors use affinity molecules as bioreceptors, such as antibodies, nucleic acids, synthetic receptors, in which the sensing principle is based upon the detection of affinity interactions between the bioreceptors and the analyte. Both the catalytic and affinity biosensors are triggered by the biomolecule recognition event between the guest analyte molecules and the host bioreceptors, and such recognition can be characterised in terms of the kinetic process with the binding constant $k_{b}$ and unbinding constant $k_{\text {off }}$ and thermodynamic process for the binding complex as shown in Figure 3.12a. Figure $3.12 \mathrm{~b}$ and $\mathrm{c}$ illustrate the affinity process with recognition and signal amplification, as well as the catalytic process with recognition by an enzyme-substrate complex and subsequent catalysis into products, respectively. Based on the transduction mechanism, biosensors are generally categorised into optical, electrochemical, calorimetric, piezoelectric, thermal and micro-mechanical biosensors. 
a

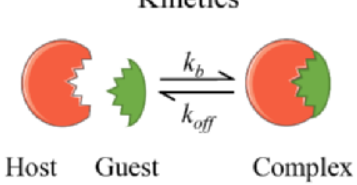

b

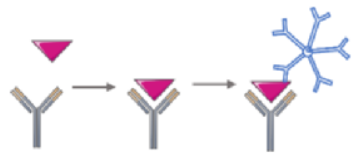

Affinity
Thermodynamics
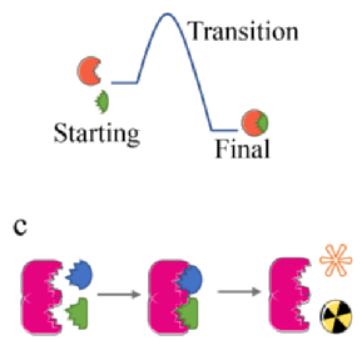

Catalytic

Figure 3.12 (a) kinetics and thermodynamics for biomolecule recognition; (b) affinity process with recognition and signal amplification by poly-clonal antibody; (c) catalytic process with recognition to enzyme-substrate complex and subsequent catalysis into products.

\subsection{Electrochemical Enzyme-based Biosensors}

Electrochemical biosensors make efficient use of bioreceptors as biosensing elements and combined with electrochemical techniques and offer low-cost and ease-of-operation with high sensitivity, extending the use of electrochemical sensors towards a large variety of biological analytes. In the field of electrochemical biosensors, much attention has been devoted to enzyme-based biosensors, immunosensors and DNA sensors ${ }^{53}$. Among these, electrochemical enzyme-based biosensors are mainly focused and discussed in this thesis.

\subsubsection{Generations of Enzyme-based Biosensors}

The mechanism of an electrochemical enzyme-based biosensor is based on a relayed enzymatic catalysis of analytes at the enzyme-based bio-electrode interface and subsequent electrochemical signal transduction at the functionalised electrode interface. Based on the different signal transduction process at the functionalised electrode interface, enzyme-based biosensors can be categorised into three so-called "generations" as shown in Figure 3.13.

In a 1st generation of enzymatic biosensor, the signal transduction is based on the measurement of the consumption of co-substrate $\left(e . g . \mathrm{O}_{2}\right)$ or the production of enzymatic product (e.g. NADH and $\mathrm{H}_{2} \mathrm{O}_{2}$ ). The 1 st generation of enzymatic biosensors has been intensively researched since the first proposal of an "enzyme electrode" based on an oxygen electrode or the detection of $\mathrm{H}_{2} \mathrm{O}_{2}$ at a platinum electrode by Leyland $\mathrm{C}$. 
Clark, in $1962^{2}$. However, the sensitivity of such enzymatic biosensors is influenced by the concentration of dissolved oxygen in the system.

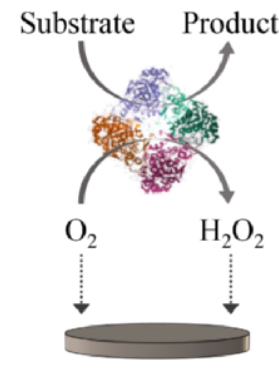

1st generation

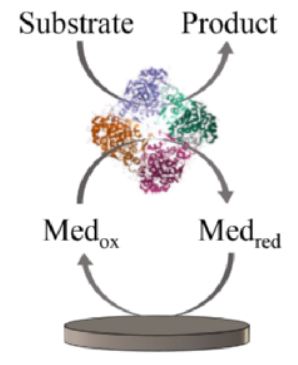

2nd generation

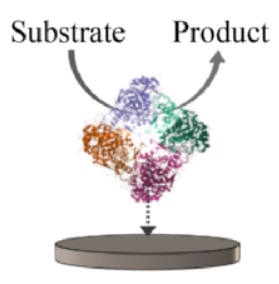

3rd generation

Figure 3.13 Schematic illustration of 1st, 2nd and 3rd enzymatic biosensors.

To eliminate the oxygen dependency impact, redox mediators such as ferrocene derivatives, ferrocyanide, dyes and quinones were applied in the 2nd generation of enzymatic biosensors to shuttle electrons between enzyme active centre and electrode surface. The mediator is usually reduced by the electron generated from the enzymatic reaction of analytes, and then is oxidised at the electrode surface. Despite it is oxygenindependence, the 2nd generation of enzymatic biosensors have limitations such as mediator leaching and a diffusion barrier of the mediator at enzyme/electrode interface.

The 3rd generation of enzymatic biosensors involves direct electron transfer (DET) between the enzyme active centre and electrode surface to generate the response without the use of other mediators and reagents. Efficient DET has been reported for many redox proteins in recent decades since the very first reports on reversible electrochemistry by cytochrome $c$ on gold and tin doped indium oxide electrodes ${ }^{54,55}$. The redox proteins or enzymes with known DET properties usually contain redox active metallo-centres in their active site, such as heme-containing cytochrome $c$ and horseradish peroxidase $(\mathrm{HRP})^{54}$, copper-containing laccase and ascorbate oxidase ${ }^{55}$, as well as iron-sulphur cluster containing ferredoxin ${ }^{56}$, or contain an organic cofactor, such as flavin-containing glucose dehydrogenase $\mathrm{e}^{57}$ and fructose dehydrogenase $\mathrm{e}^{58}$ as well as quinone-containing lactate dehydrogenase $\mathrm{e}^{59}$. However, deeply embedded active redox centres and denaturation of enzymes on flat electrode surfaces may influence the DET efficiency, although it has been argued that this could potentially be improved by the use of nanomaterials. 


\subsubsection{Immobilisation of Enzymes}

The two key factors for the development of an electrochemical enzyme-based biosensor with high performance are the biosensing layer with immobilised enzyme and the transduction layer with a functionalised electrode, in which the latter is discussed in detail in section 3.2. The immobilisation of enzyme is important from the aspects of loading amount, biological activity and structure retention after immobilisation, longterm storage and operation stability, as well as the immobilisation stability to minimise the leakage during operation, which determines the sensitivity and stability of a biosensor. Other considerations for the immobilisation of enzyme are reproducibility, cost and difficulty of the whole process. Enzyme immobilisation techniques can be classified as adsorption, entrapment, cross-linking, covalent coupling and bioaffinity interaction ${ }^{60}$.

Adsorption is a simple approach for immobilisation involving physical adsorption via week interactions (e.g. hydrogen bond, hydrophobic and van der Waals attractions) and electrostatic attractions (e.g. layer-by-layer (LBL)) for opposite-charged enzyme molecules, which is favourable for activity retention without the disruption of subunits of enzymes. No modification is needed for the enzyme in this approach, but enzymes tend to leak during operation because of the weak interactions. Entrapment or encapsulation provides another non-covalent approach for enzyme immobilisation into a matrix, which is favourable for the distribution of the enzyme molecules inside the matrix without disruption and denaturation. A variety of matrices have been used for encapsulation of enzymes, such as organic polymer including CPs, sol-gels or hydrogels and biomacromolecules including liposome and polysaccharide. However, the additional encapsulation layer may cause a huge diffusion barrier, thus reduce the sensitivity and response time. To address this issue, efforts have been made to produce 3D enzymatic electrodes with hierarchically nanostructured pores for well distributed enzyme, fast substrate diffusion, efficient enzyme-substrate reaction and electrical transfer with the electrode ${ }^{61-63}$.

The covalent coupling approach forms strong covalent bonds between the enzymes and the electrode material surface. The process usually involves the initial activation of the functional groups on the electrode surface by chemical reagents, followed by enzyme coupling to the activated substrate and final removal of the excess and unbound enzyme molecules. Carboxylic acid groups and amino groups are the most commonly used functional groups for covalent coupling of biomolecules. Covalent coupling could improve the uniformity and distribution of the enzyme molecules on the electrode surface with decreased aggregation and leakage. Similar to covalent coupling, the crosslinking approach allows the strong chemical binding of enzyme molecules with each other or with other inert biomolecules (e.g. bovine serum albumin) with the assistance 
of a cross-linker, such as glutaraldehyde. In addition, enzyme immobilisation can be realised via bioaffinity interactions such as biotin-avidin, lectin-carbohydrate and metal cation-chelator interactions. These approaches provide control of the orientation of enzyme molecule in order to decrease enzyme deactivation and/or active site blocking ${ }^{60}$.

\subsubsection{Electrochemical Enzyme-based Lactate Biosensors}

Lactate is an important metabolite from the anaerobic glycolytic pathway resulting from the converting of pyruvate to lactate by the enzyme lactate dehydrogenase (LDH). The physiological level of lactate in human body reflects the energy-related metabolism, which is the prerequisite for cellular function and its alteration can be used as a key biomarker for pathophysiological diagnosis, such as ischemia, cellular fatigue, oxygen deficiency, liver disease and cancers ${ }^{64}$. In addition, the level of lactate in the body can be used as an indicator for health monitoring in sport and military applications to evaluate the fitness, intensity and duration of the activities, since a high level of lactate causes lactic acidosis. The concentration of lactate in different biofluids are variable as shown in Table 3.2 which is summarised from previously reported papers ${ }^{65,66}$. The monitoring of lactate from arterial blood provides accurate quantification of lactate level as the "gold standard", but the invasive sampling is painful and needs well-trained personnel. Thus, it is difficult to commercialise such lactate monitoring devices. Alternatively, monitoring from other body-fluids is potentially promising for noninvasive monitoring of lactate using, for example, tears, saliva and sweat. However, the correlation between blood lactate and other body-fluid lactate levels remains doubtful, especially for sweat. Even so, continuing extensive research reveals a positive correlation for the development of non-invasive monitoring of lactate during physical exercise as opposed to disease diagnosis ${ }^{67}$.

Table 3.2 Summary of lactate level in different biofluids.

\begin{tabular}{llllll}
\hline & Blood $/ \mathrm{mM}$ & $\begin{array}{l}\text { Interstitial } \\
\text { fluid } / \mathrm{mM}\end{array}$ & Tears $/ \mathrm{mM}$ & Saliva/mM & Sweat $/ \mathrm{mM}$ \\
\hline Concentration & $\begin{array}{l}\text { Rest } 0.5-2, \\
\text { Exercise } \sim 25\end{array}$ & $1.9-2.2$ & $1-5$ & $0.1-2.5$ & $>20$ \\
\hline
\end{tabular}

The electrochemical lactate biosensors provide a simple and fast device to monitor lactate levels. Such biosensors rely on the immobilisation of catalytic enzyme on the electrode surface. Lactate dehydrogenase (LDH) is an important dehydrogenase that relies on the cofactors of $\mathrm{NADH}$ and $\mathrm{NAD}^{+}$according to the following reaction.

$$
\begin{gathered}
\text { Lactate }+\mathrm{NAD}^{+} \rightarrow \text { pyruvate }+\mathrm{NADH} \text { in presence of } \mathrm{LDH} \\
\mathrm{NADH} \rightarrow \mathrm{NAD}^{+}+\mathrm{H}^{+}+2 \mathrm{e}^{-}
\end{gathered}
$$


In LDH-based lactate biosensors, lactate is catalytically oxidised into pyruvate, while the co-existing $\mathrm{NAD}^{+}$is reduced to NADH. Then the quantification of lactate concentration is based on the electrochemical oxidation of NADH into $\mathrm{NAD}^{+}$at the transducer interface.

Lactate oxidase (LOx), a flavin mononucleotide-dependent alpha hydroxyl acid oxidising enzyme, is another widely used enzyme for the fabrication of electrochemical lactate biosensors. According to the following enzymatic reactions, the flavin adenine dinucleotide (FAD) cofactor in the active centre of LOx oxidise lactate into pyruvate with resulting $\mathrm{FADH}_{2}$, which is then reduced back to $\mathrm{FAD}$ in the appearance of dioxygen $\left(\mathrm{O}_{2}\right)$ with the production of $\mathrm{H}_{2} \mathrm{O}_{2}$. Under a sufficient applied potential, the production of $\mathrm{H}_{2} \mathrm{O}_{2}$ or consumption $\mathrm{O}_{2}$ can be transduced into an electrical signal via an electrode in the 1 st generation of enzymatic electrode.

$$
\begin{gathered}
\text { Lactate }+\mathrm{LOx}(\mathrm{FAD}) \rightarrow \text { pyruvate }+\mathrm{LOx}\left(\mathrm{FADH}_{2}\right) \\
\text { LOx }(\mathrm{FADH} 2)+\mathrm{O}_{2} \rightarrow \mathrm{LOx}(\mathrm{FAD})+\mathrm{H}_{2} \mathrm{O}_{2} \\
\mathrm{H}_{2} \mathrm{O}_{2} \rightarrow \mathrm{O}_{2}+2 \mathrm{H}^{+}+2 \mathrm{e}^{-} \\
\text {Or } \mathrm{O}_{2}+4 \mathrm{H}^{+}+4 \mathrm{e}^{-} \rightarrow 2 \mathrm{H}_{2} \mathrm{O}
\end{gathered}
$$

The oxidation of NADH produced from $\mathrm{LDH}$ and $\mathrm{H}_{2} \mathrm{O}_{2}$ from LOx usually needs a high overpotential, resulting in electrochemical interferences and electrode fouling. Therefore, different strategies have been developed to reduce the overpotential and increase the reaction kinetics and thus increase the sensitivity; for instance, the use of redox mediators, carbon-based nanomaterials, metal complexes and conducting polymers to deliver larger electrode surface area, higher conductivity, and higher catalytic activity. In addition, the biocompatibility of the functionalised electrode is another consideration when seeking to increase the electrochemical performance by increasing the amount and stability of immobilised enzyme molecules. In Paper IV and $\mathbf{V}$ in this thesis, $\mathrm{LDH}$ and LOx were used to fabricate a lactate biosensor based on $\mathrm{NADH}$ and $\mathrm{H}_{2} \mathrm{O}_{2}$ transduction, respectively.

\subsubsection{Electrochemical Enzyme-based Glucose Biosensors}

Diabetes mellitus is one of the most prevalent chronic diseases with the metabolic disorder of blood glucose levels resulted from insufficient insulin production or secretion. Although it is not clear how to cure diabetes, patients with diabetes are recommended to monitor their blood glucose levels several times per day, and this is a key part for diabetes self-management. Blood glucose monitoring is the gold-standard method for glucose sampling and the normal range of blood glucose is $4.4-6.6 \mathrm{mM}$ $(80-120 \mathrm{mg} / \mathrm{dL})^{68}$. However, self-monitoring of blood glucose by the commonly used "finger prick" test is invasive and provides only a temporal value. In recent years, much 
effort has been focused on the development of continuous glucose monitoring (CGM) in interstitial fluid and other proof-of-concept studies for glucose level detection in sweat, tears and saliva in a minimally-invasive or noninvasive manner ${ }^{69}$.

Most glucose detection devices rely on enzyme-based amperometric biosensors to measure glucose concentrations, in which GOx established the first basic principle for the electrochemical enzymatic detection of glucose. GOx is a typical oxidoreductase with two bound flavin adenine dinucleotide (FAD) as redox prosthetic group. The whole enzymatic reactions and signal transduction for the fabrication of the 1st generation of biosensors are shown as the following reactions. The conversion of glucose into gluconolactone by GOx involves the transfer of two protons and two electrons to coenzyme FAD with resulting $\mathrm{FADH}_{2}$ which is then reduced back to FAD by $\mathrm{O}_{2}$ accompanying the production of $\mathrm{H}_{2} \mathrm{O}_{2}$. The depletion of $\mathrm{O}_{2}$ or liberation of $\mathrm{H}_{2} \mathrm{O}_{2}$ can be measured electrochemically to assess the glucose level.

$$
\begin{gathered}
\text { Glucose }+\mathrm{GOx}(\mathrm{FAD}) \rightarrow \text { Gluconolactone }+\mathrm{GOx}\left(\mathrm{FADH}_{2}\right) \\
\text { GOx }\left(\mathrm{FADH} \mathrm{F}_{2}\right)+\mathrm{O}_{2} \rightarrow \mathrm{GOx}(\mathrm{FAD})+\mathrm{H}_{2} \mathrm{O}_{2} \\
\mathrm{H}_{2} \mathrm{O}_{2} \rightarrow \mathrm{O}_{2}+2 \mathrm{H}^{+}+2 \mathrm{e}^{-} \\
\mathrm{Or} \mathrm{O}_{2}+4 \mathrm{H}^{+}+4 \mathrm{e}^{-} \rightarrow 2 \mathrm{H}_{2} \mathrm{O}
\end{gathered}
$$

Alternatively, glucose dehydrogenase (GDH) has been used for the fabrication of amperometric glucose biosensors that could be operated at lower potentials and eliminate the oxygen deficiency in GOx-based 1st generation glucose biosensors. NADdependent GDH is one of the subclasses of GDH that can be used for detection of glucose without using oxygen as a cosubstrate, which relies on $\mathrm{NAD}^{+}$acting as a cofactor and electrochemical oxidation of the NADH product as the following reactions ${ }^{70}$.

$$
\begin{aligned}
\text { Glucose }+\mathrm{NAD}^{+} \rightarrow & \text { Gluconolactone }+\mathrm{NADH} \text { in presence of GDH } \\
& \mathrm{NADH} \rightarrow \mathrm{NAD}^{+}+\mathrm{H}^{+}+2 \mathrm{e}^{-}
\end{aligned}
$$

In addition, pyrroloquinoline quinone (PQQ)-dependent GDH is another important enzyme adopted by industries for glucose sensor systems, which employ tightly bound PQQ as coenzyme as the following reactions ${ }^{68,71,72}$.

$$
\text { Glucose }+ \text { PQQ(ox) } \rightarrow \text { Gluconolactone }+ \text { PQQ(red) }
$$


Chapter 3 


\section{Chapter 4 Conducting Polymers}

Conducting polymers (CPs), or intrinsically conducting polymers (ICPs), are a class of functional organic polymers with $\pi$-conjugated double bonds along the polymer chains that could conduct electricity. The high conjugation of the polymer chain and its doping/de-doping process endow the CPs with new unique physicochemical properties, such as electronic/ionic conductivity and electrochemical reversibility. In addition, the mechanical flexibility originating from the intrinsically polymeric nature makes the CPs promising for electrochemical sensing and biosensing. In this chapter, the tailoring of CPs for sensor and biosensor applications is discussed in detail from the aspects of modulation of the $\mathrm{CP}$ surface charge, creating $\mathrm{CP}$ micro/nano-structures, introducing functional groups via $\mathrm{CP}$ derivatives and fabrication of $\mathrm{CP}$ composites with additional physicochemical properties.

\subsection{Basic Characteristics of CPs}

\subsubsection{Development of CPs}

Research in the field of CPs can be traced back to the middle of the 19th century with the chemical polymerisation of aniline by Letheby, but only since Shirakawa and Heeger/MacDiarmid's groups discovered the metallic conductivity of crystalline polyacetylene (PA) doped with halogen (p-type dopants) in 1977, has the field of CPs attracted much attention from both of the academic and industrial communities and it has subsequently developed rapidly ${ }^{73}$. In 2000, Alan J. Heeger, Alan G. MacDiarmid, and Hideki Shirakawa were awarded a Nobel Prize in Chemistry for their contribution to "the discovery and development of conductive polymers." The last few decades have witnessed the development and application of CPs in different fields, with more than 25 classes of CPs established. Among them, polypyrrole (PPy), polyaniline (PANI), poly(thiophenes) (PTs) and poly(3,4-ethylenedioxythiophene) (PEDOT) are the most extensively studied to date from both academic and industrial points of view. Figure 4.1 illustrates the structure of several representative CPs. 


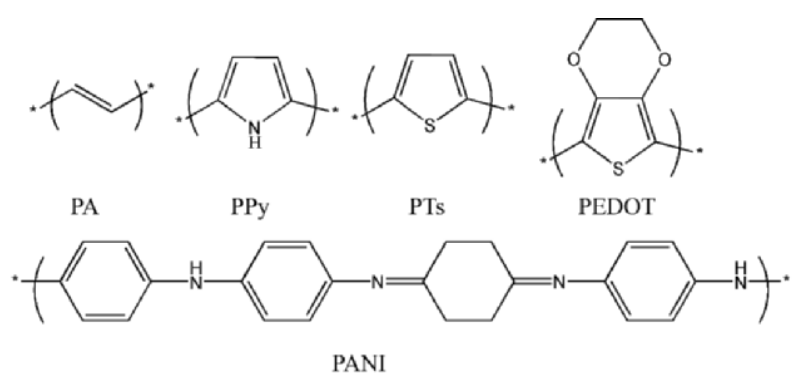

Figure 4.1 Structures of several representative CPs.

\subsubsection{Properties of CPs}

CPs serve as the preferred active and conductive material in many fundamental and applied electronic devices owing to their inherited mechanical flexibility as organic polymers, as well as their new fascinating properties in electronics including electronic conductivity as a conductor, as well as ionic conductivity and redox reversibility. The neutral state of CPs is insulating. During the chemical or electrochemical oxidation of the $\mathrm{CPs}$, electrons are removed from the valence band, i.e. p-type doping, leading to delocalised charge carriers alongside the positively-charged polymer chain which is compensated with negatively-charged counterions. In contrast, n-type doping occurs when the polymer chain is reduced chemically or electrochemically with a negativelycharged backbone that is compensated by the inserted positively-charged counterions. The CPs studied in the thesis are p-type doped CPs.

The doping process is reversible. For p-type CPs, the dedoping process occurs when reducing the CPs to undoped state without the degradation of the $\mathrm{CP}$ backbone. The reversible doping/dedoping process imparts the CPs with a variety of promising physicochemical properties as shown in Figure 4.2. In the doped sate, CPs possess electrical conductivity, and the conductivity can be tuned by the doping level, covering three broad conductivity classifications of materials including insulators, semiconductors and metals. During the doping/dedoping process, the dopant can move into and out of the CP matrix, making the CPs ionically conductive. The transport of ions and solvent into and out of the $\mathrm{CP}$ matrix lead to the swelling or shrinking of $\mathrm{CPs}$ and thus the corresponding physical and volumetric strains. Although such volumetric change is favourable of the employment for soft actuators, it inevitably leads to low cycling life for other electrochemical applications. Redox reversibility is another electrochemical property of CPs imparted from doping/dedoping process, during which $\mathrm{CP}$ is oxidised in the doping process and reduced in the dedoping process. In addition, electrochemical doping/dedoping also influences the optical properties and transparency of CPs. Using PEDOT on the ITO glass as the model in Figure 4.2, PEDOT in the 
oxidised state (doped) displays a light blue color with high transparency, while it shows as dark blue in the reduced state (dedoped).

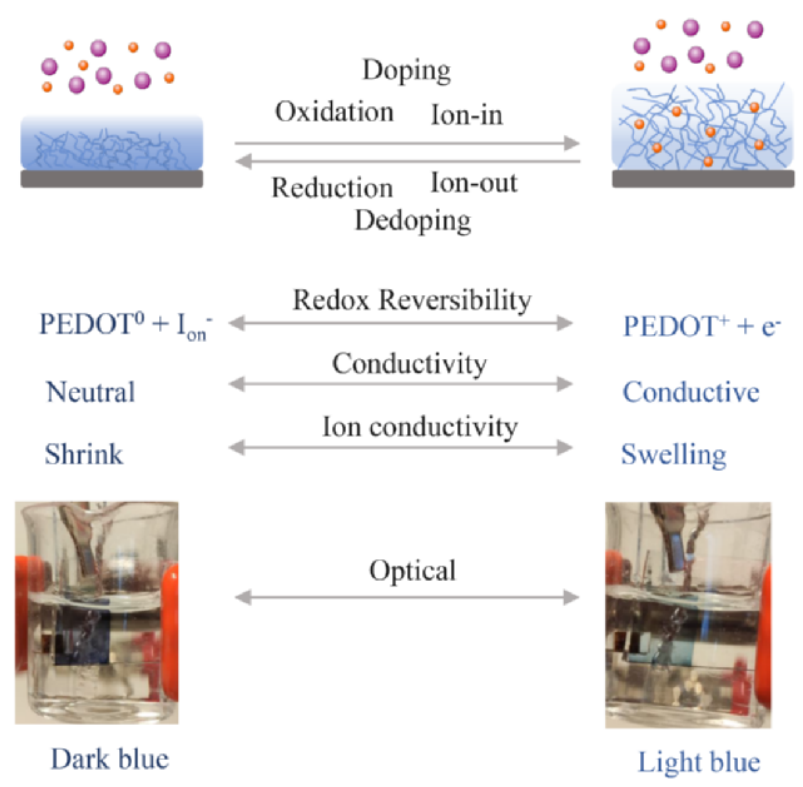

Figure 4.2 Schematic diagram illustrating the reversible doping/dedoping of CPs using PEDOT as a model.

Apart from these electrical and optical properties, CPs are inexpensive and stable. In addition, the rich synthetic chemistry allows the fine tailoring of their molecular structure with versatile functional groups for specific applications. What is more, their intrinsically mechanical flexibility facilitates the easy formation of a tenacious film on the substrate and further usage for soft electronic devices.

\subsubsection{Polymerisation of CPs}

Polymerisation is a process for the formation of polymer chains or three-dimensional networks from their monomers, dimers or oligomers. As shown in Figure 4.3 using PEDOT as the model, the polymerisation of CPs usually proceeds via: 1) initial oxidation of the monomers into radical cation; 2) coupling where a radical cation attacks another monomer molecules to produce a dimer radical cation or radical-radical coupling to a radical di-cation and then deprotonate and oxidise to a dimer radical cation, depending on the different oxidation approaches applied; 3 ) propagation of the polymer chains $^{73}$. 
Chemical and electrochemical approaches are commonly used for oxidative polymerisation of CPs from their monomers. In chemical polymerisation, chemical oxidants, such as iron (III) chloride $\left(\mathrm{FeCl}_{3}\right)$, iron (III) tosylate (Fe(TOS)) and ammonium persulphate $\left(\left(\mathrm{NH}_{4}\right)_{2} \mathrm{~S}_{2} \mathrm{O}_{8}\right)$, are applied to initial the oxidation of the monomers. Chemical polymerisation allows the deposition of CPs on a variety of substrates, especially for non-conductive substrates, and the bulk synthesis in large quantities, such as the commercially available PEDOT:PSS suspension ${ }^{74}$. Alternatively, electrochemical polymerisation is carried out by employing a potential or current with potentiodynamic, potentiostatic or galvanostatic techniques on a conductive substrate. The electropolymerisation usually follows: 1) instantaneous 2D mechanism with oligomer growth on the electrode surface to a compact film; 2) progressive 3D nucleation and growth with branched CPs formation ${ }^{75}$. The advantages of this approach are controllable polymerisation amount by controlling the time, current and potential, tunable morphology by adjusting the electrolyte, template and temperature etc., capability to incorporate different dopant or composites, co-polymerisation of different monomers and sequential deposition of different CP films.

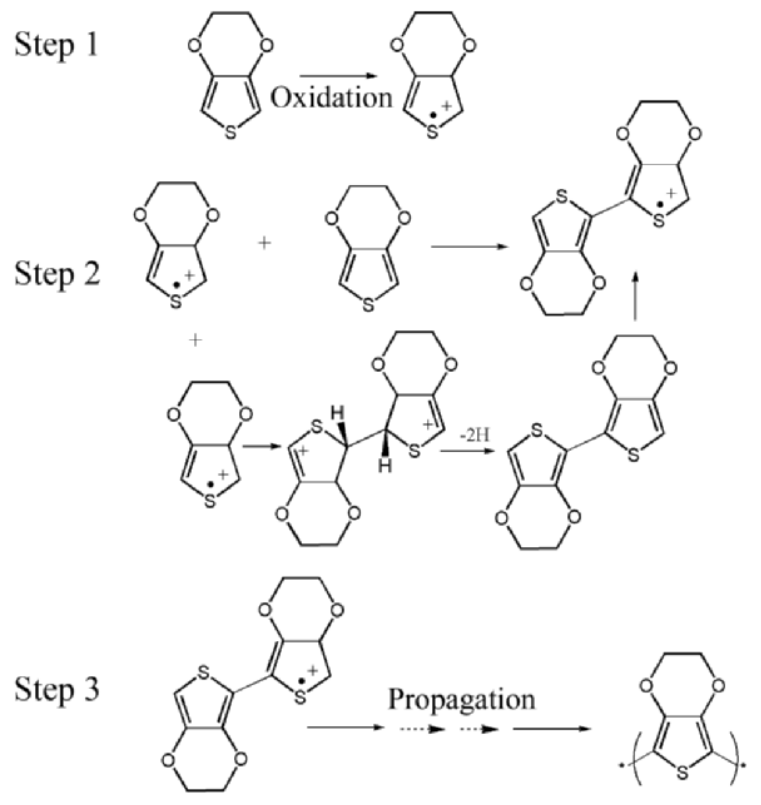

Figure 4.3 CPs polymerisation process using PEDOT as the model with initial oxidation for radical cation (step 1), coupling of dimer radical cation (step 2) and propagation of the CP chain (step 3). 


\subsubsection{Applications of CPs}

Based on their unique electrical and ionic conductivity, optical and mechanical properties, CPs have demonstrated their promising potential in a wide range of applications. Their specific optical and photonic properties enable the applications of CPs in organic light-emitting diodes and electrochromic displays ${ }^{76}$. In the field of energy conversion and storage fields, much effort has been devoted to their application in solar cells, electrocatalysis, fuel cells, batteries and supercapacitors ${ }^{77}$. The electrical and ionic conductivity of CPs can be exploited for the fabrication of electronic devices including OFET, sensors, biosensors and actuators, as well as many biomedical applications, such as tissue engineering, neural interface and drug delivery $e t c^{78}$.

\subsection{PEDOT}

As a derivative of thiophenes, 3,4-ethylenedioxythiophene (EDOT) was first synthesised at the Bayer AG research laboratories in the 1980s because the oxygensubstituted thiophenes can increase the stability in its polymeric form and eliminate the undesirable $\alpha, \beta$-coupling and $\beta, \beta$-coupling ${ }^{79}$. Since then, PEDOT has attracted considerable attention because of its excellent stability, reversible doping state, transparency, high conductivity and electrochemical properties.

\subsubsection{Incorporation of Dopant into PEDOT}

Dopants or counterions are introduced into the PEDOT matrix during the oxidative polymerisation. In chemical oxidative polymerisation, the choice of the dopants is quite limited because the dopants usually originate from the oxidants. However, a large variety of dopants can be doped into electropolymerised PEDOT as long as the dopants carry negative charge and can be stably dispersed in the reaction solvents, ranging from small molecules including $\mathrm{Cl}^{-}, \mathrm{ClO}_{4}^{-}, \mathrm{NO}_{3}{ }^{-}$, tosylate and carboxylate, polyelectrolytes including poly(styrene sulfonate) (PSS), poly(acrylic acid) (PAA) and Nafion, to biomolecules and biomacromolecules including hemin, hyaluronic acid, nucleic acid and enzyme etc.

The incorporation of dopants into the PEDOT matrix is an important prerequisite for obtaining a conductive PEDOT owing to their important role in doping level, doping affinity and polymerisation efficiency. For instance, Spanninga et a ${ }^{80}$ reported that there exists an affinity trend for different anions with thiosulfate dominating over all other anions as PEDOT counterions, such as $\mathrm{Cl}^{-}$and $\mathrm{ClO}_{4}^{-}$, but the factor that determinates PEDOT counterion affinity remains unclear. Besides the counterion affinity, co-existing counterions in the solution during polymerisation may be co-doped into the PEDOT matrix with improved polymerisation efficiency. This phenomenon was demonstrated 
in Paper II by the integration of dopants with different affinity (i.e. citrate as a lower affinity dopant and $\mathrm{ClO}_{4}^{-}$as a high affinity dopant). As shown in Figure 4.4, the electropolymerisation of PEDOT with citrate as the dopant (PEDOT:C) shows low polymerisation efficiency with a high onset oxidation potential $(1.0 \mathrm{~V})$, no apparent increase in currents with the increasing of cycling numbers, and loosely-populated PEDOT particles. In contrast, the $\mathrm{ClO}_{4}^{-}$dopants facilitated the polymerisation of PEDOT (PEDOT:P) with decreased onset oxidation potential to $0.9 \mathrm{~V}$, notably increased current upon successive cycles and compact film with cauliflower-like structure. To introduce functionality onto the PEDOT interface, citrate with carboxylate groups was co-doped into PEDOT (PEDOT: $\mathrm{L}_{2} \mathrm{C}_{1}$ ) with the assistance of $\mathrm{ClO}_{4}{ }^{-}$with high polymerisation efficiency, resulting in a dense compact film with a well-distinguished and homogeneously distributed globular structure on the electrode surface.
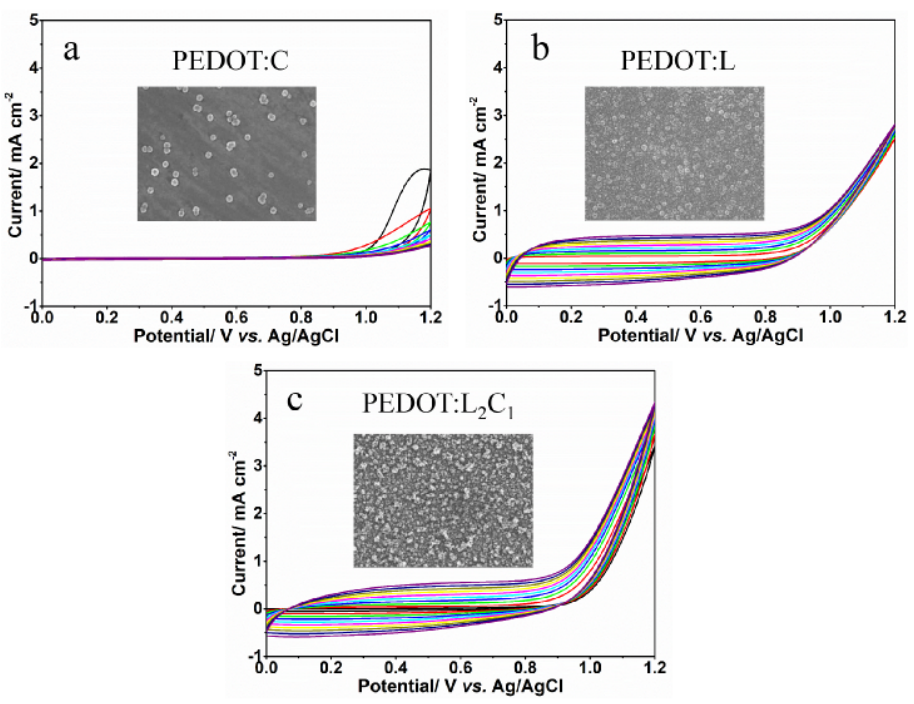

Figure 4.4 Potentiodynamic polymerisation of PEDOT using citrate (a, PEDOT:C), $\mathrm{ClO}_{4}^{-}$(b, PEDOT:L), citrate and $\mathrm{ClO}_{4}^{-}$(c, PEDOT: $\mathrm{L}_{2} \mathrm{C}_{1}$ ) as dopants, insets are the corresponding SEM image of the electropolymerised PEDOT on Au-Si electrode surface.

The dopants incorporated into the PEDOT matrix endow it with new properties, such as charge, solubility and processability. Intrinsic PEDOT presents low water solubility and processability. The introduction of excess negative charge by dopants can improve the colloidal stability of PEDOT in water solution. For instance, PSS is used as a counterion to prepare water processable PEDOT:PSS (Figure 4.5), since PSS can compensate the positive charges on the PEDOT backbone, as well as facilitate the water dispersibility 
of the PEDOT segments by its high hydrophilicity. In addition, the effect of dopants serving as a soft-template to tailor the PEDOT morphology is discussed in detail in section 4.3. What is more, some other functional materials are employed to be incorporated into the PEDOT matrix as composites, which is discussed in detail in section 4.5.

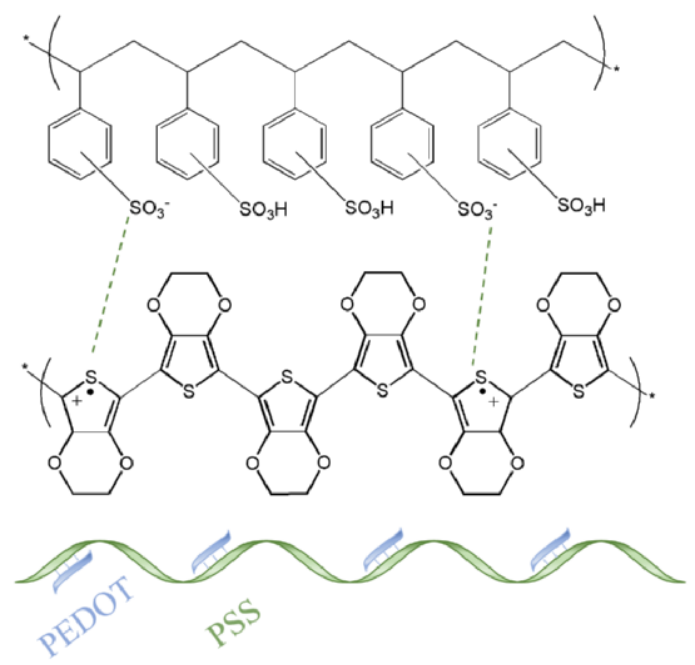

Figure 4.5 Structure of commercially available PEDOT:PSS.

The stability of the dopants incorporated into PEDOT matrix and their influence on the surface and bulk properties are also important for the choice of dopants. The employment of small-sized anions as dopants for PEDOT, such as tosylate and perchlorate, is favourable for ordered crystalline structure and thus high conductivity ${ }^{81}$. However, the dopants undergo leakage from the matrix and replacement by the anions in the bulk solution during doping/de-doping process ${ }^{82,83}$. The doping of large-sized molecules could improve the stability, however, they may interrupt the surface morphology and decrease the conductivity owing to the large excess of insulting dopants inside the PEDOT ${ }^{82,84}$.

\subsubsection{Modulating PEDOT Surface Charge in Sensing and Biosensing}

Charge-charge interactions are found to be useful in many fields, such as surface coating, the ink industry, separations, drug release, environmental treatments, LBL fabrication and analytical chemistry. The charge property on a CME surface also contributes to the chemical sensing and biosensing of analytes in the manner of: 1) electrostatic accumulation of opposite-charged analyte molecules; 2) repelling the same-charged interference molecules; 3 ) increasing the anti-fouling ability towards intermediates or 
products; 4) immobilisation of biosensing recognition elements. The introduction of different charges, namely positive charge and negative charge, can be realised with different materials including polyelectrolytes ${ }^{85}$, ligands ${ }^{86}$, metal oxide ${ }^{87}$ (e.g. titanate, silica), carbon-based nanomaterials ${ }^{88}$ (e.g. graphene oxide) and so on.

Compared to these conventional materials, PEDOT provides a versatile platform for the fabrication of a charged electrode surface with high conductivity. Negatively charged sensing surfaces can be fabricated on the electrode surface by polymerising PEDOT with excess negatively-charged dopants, such as PSS and Nafion ${ }^{89}$. On the other hand, the surface charge can be introduced directly onto the PEDOT interface by utilising EDOT derivatives bearing charged functional groups during the polymerisation process, such as EDOT-COOH ${ }^{90,91}$. Moreover, post-treatment of the PEDOT modified electrode surface with charged surfactants or polyelectrolytes can also introduce charge on PEDOT surface. For instance, Atta et al..$^{92}$ demonstrated the deposition of a monolayer of sodium dodecyl sulfate on PEDOT to produce a negatively-charged electrode surface to facilitate the selective determination of DA in presence of high concentrations of AA and UA. However, the post-treated non-conducting layer on the PEDOT sensing surface would influence the conductivity and charge transfer thus resulting in low sensitivity. Therefore, the modulation of charge effect directly on the PEDOT backbone/surface with high conductivity is desirable. In this thesis, two different charged PEDOT, i.e. positively-charged PEDOT colloidal microparticles (Paper I) and negatively-charged carboxylic acid group functionalised PEDOT (PEDOT:COOH) (Paper II), were developed and used for chemical sensing of negatively-charged NADH and positivelycharged DA, respectively, showing the corresponding electrostatic effect for the attraction of targeted analytes accompanied by repulsion of electrochemical interferents (e.g. AA and UA).

The electrochemical behaviours of positively-charged or negatively-charged electrode surfaces can be investigated using two differently charged redox probes (i.e., negativelycharged $\mathrm{Fe}(\mathrm{CN}) 6^{3-/ 4-}$ and positively-charged $\mathrm{Ru}\left(\mathrm{NH}_{3}\right) 6^{2+/ 3+}$ to prove the surface charge effect. As shown in Figure 4.6, negatively-charged $\mathrm{Fe}(\mathrm{CN}) 6^{3-/ 4-}$ molecules tend to diffuse to a positively-charged electrode and accumulate at the surface owing to the electrostatic attraction effect, while such an electrode is prone to repel the same charged $\mathrm{Ru}\left(\mathrm{NH}_{3}\right)_{6}{ }^{2+/ 3+}$ molecules by the electrostatic repulsion effect. On the contrary, a negatively-charged electrode surface facilitates the accumulation of positively-charged $\mathrm{Ru}\left(\mathrm{NH}_{3}\right)_{6}{ }^{2+/ 3+}$ and repel the same charged $\mathrm{Fe}(\mathrm{CN})_{6}{ }^{3-/ 4-}$ molecules. Such electrostatic attraction and repulsion of charged analyte molecules can be observed through CVs by comparison of their redox peak potentials, redox peak potential difference and redox peak currents. 


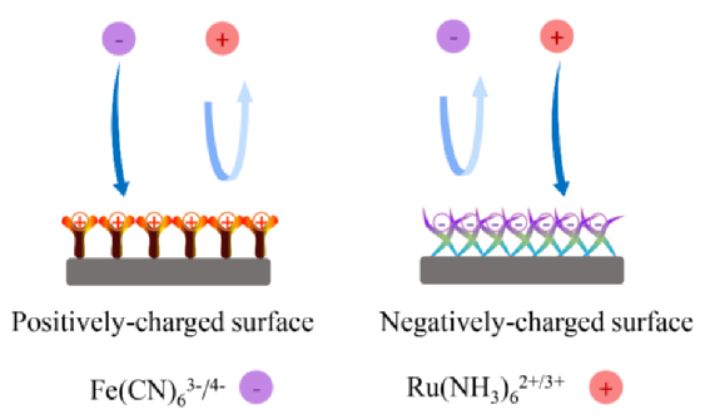

Figure 4.6 Diagram of the attraction or repulsion of negatively-charged $\mathrm{Fe}(\mathrm{CN}) 6^{3-14-}$ and positively-charged $\mathrm{Ru}\left(\mathrm{NH}_{3}\right)_{6}{ }^{2+/ 3+}$ at different charged electrode surfaces.

\subsection{PEDOT Nanostructures}

\subsubsection{Nanostructure Features}

The rapid development of nanotechnology and material science makes it possible to design and fabricate materials with controlled size and morphology on a micro and nano scale. The fabrication of nanostructures with different dimensions, including 0D (e.g. nanoparticles), 1D (e.g. nanotubes, nanorods and nanowires), 2D (e.g. thin film and nanolayers) and 3D (complex of multi-level of nanostructures) can be realised in two directions, i.e. bottom-up using atoms or molecules as the building blocks for the creation of complex structures, and top-down methods via removing or division of bulk materials into micro/nano parts, in which self-assembling, 3D printing, etching, lithography, micromachining, and template-orientation etc. techniques are commonly used during the process.

Nanostructured materials, therefore, gain new or improved physicochemical, optical, mechanical and electrochemical properties compared with conventional materials and their bulk counterparts. Nanostructured materials usually possess high surface area to volume ratio, which are favourable for the communication between the analytes with the structured electrode surface for signal transduction. Especially in electrochemistry, the electrochemical kinetics at the bulk electrode surface are sluggish with slow electron transfer due to the low specific surface area caused by hysteresis.

\subsubsection{PEDOT Nanostructures}

The precise control and tuning of PEDOT structure and morphology can be realised by bottom-up routes during the synthesis process or by post-treatment. In bottom-up routes, the polymerisation of PEDOT materials is commonly synthesised in the form of powder or film on substrate surface via chemical and electrochemical approaches. Template- 
orientation provides versatile potentials for the fabrication of PEDOT nanostructures in a manner of a straightforward and efficient route for large-scale synthesis. The employment of template can be divided into hard-template and soft-template. With the assistance of a template, various well-defined CP structures, including nanospheres ${ }^{93}$, nanowires ${ }^{94}$, nanotubes ${ }^{95}$, and nanoarrays ${ }^{96}$ etc., can be designed and synthesised. Figure 4.7 illustrates some examples using hard-templates or soft-templates for nanostructures.

a)

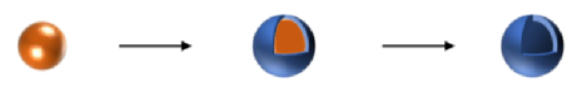

b)

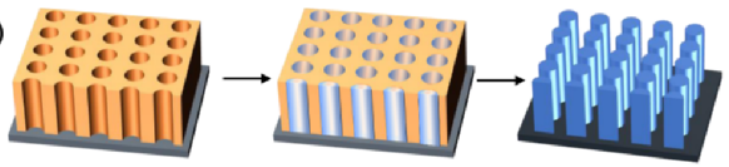

c)

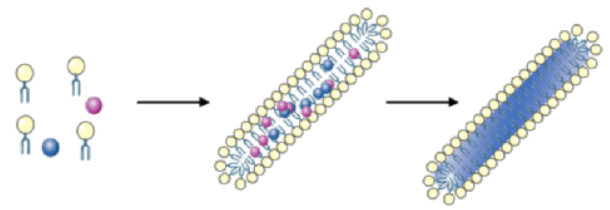

Figure 4.7 Schematic of nanostructured PEDOT fabrication process with (a) nanospheres as hard-template with core-shell structure and final hollow nanospheres, (b) anodised alumina oxide as hard-template with inner nanorods and final nanoarray, (c) sodium bis(2-ethylhexyl) sulphosuccinate as soft-template with reversedmicroemulsion for nanorod.

In hard-template synthesis, a physical template as a scaffold is applied for the chemical or electrochemical deposition of PEDOT, and then post-treatment is implemented to remove the template. The use of nanoparticles and nanorods as template leads to a coreshell structure and final hollow nanocapsules or nanorods by the removal of hardtemplate, while the use of nanosized channels results in nanowires, nanorods or nanotubes. The size and structure of the resulting CP can be tailored according to the design of the hard template such as mesoporous silica ${ }^{97}$ and anodised alumina oxide ${ }^{98}$, while template removal may need complex and harsh conditions and destroy the structure.

In Paper I, we demonstrated the fabrication of processable PEDOT colloidal microparticles (PEDOT CMs) by hard-template-assisted polymerisation and these were then utilised as building blocks for the fabrication of hierarchically-structured electrodes with a larger accessible electroactive surface. As shown in Figure 4.8, calcium 
carbonate $\left(\mathrm{CaCO}_{3}\right)$ microparticles with narrow size distribution and porosity were employed as the hard-template to assist the polymerisation of PEDOT. The removal of the $\mathrm{CaCO}_{3}$ template by the mild reagent ethylenediaminetetraacetic acid disodium $(\text { EDTA-Na })_{2}$ resulted in positively-charged and porous PEDOT microparticles suspended in aqueous solution, which were used as building blocks for the fabrication of hierarchically-structured electrodes. Similar to $\mathrm{CaCO}_{3}$, a 3D porous architecture with hierarchically interconnected graphene structure was used as the hard-template for electropolymerisation of PEDOT in Paper V. PEDOT was deposited alongside the porous graphene with well-maintained porous structure. There is no need to remove the template owing to its high conductivity. Reversely, PEDOT could reinforce the mechanical stability of the porous graphene structure as a conductive polymeric binder.

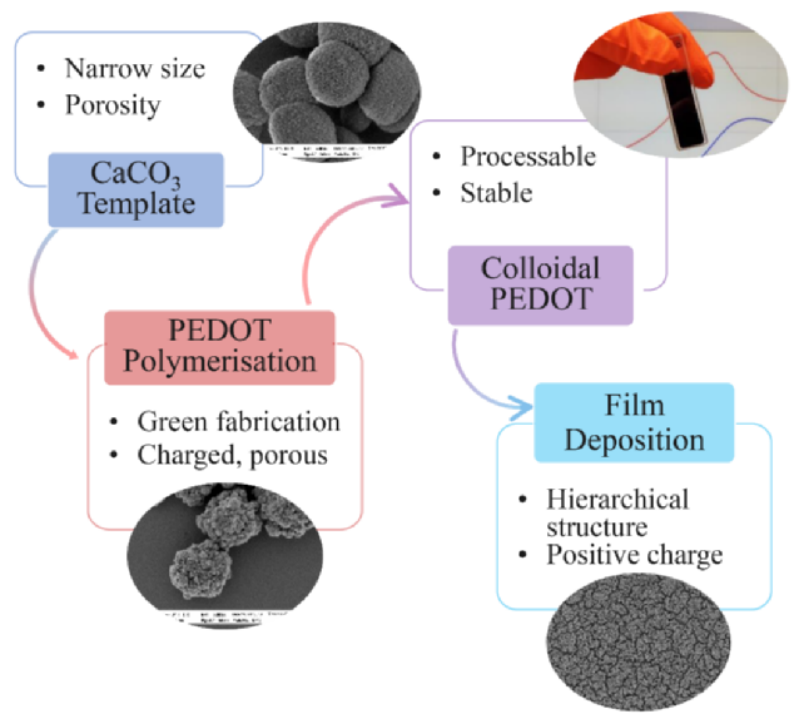

Figure 4.8 Schematic diagram of colloidal PEDOT microparticles fabrication process using porous $\mathrm{CaCO}_{3}$ as hard-template and its employment for film deposition to a hierarchical and positively-charged interface.

Soft-templates, typically consisting of a surfactant, are a simple but versatile route for PEDOT nanoparticles, nanofibres and nanotubes growth via a self-assembly process during microemulsion or reversed-microemulsion polymerisation, such as the sodium bis(2-ethylhexyl) sulphosuccinate for nanorod ${ }^{99}$. The dopant or the dimers can also serve as a self-template for PEDOT growth, usually defined as template-free route ${ }^{100}$. The soft-template or template-free approaches rely on the self-assembly of monomers and oligomers via non-covalent interactions, such as hydrogen bonds, van der Waals forces, $\pi-\pi$ stacking interaction and so $\mathrm{on}^{101}$. Unlike the chemical polymerisation of PEDOT in 
the appearance of hard-template, the morphology and size for soft-template and template-free routes are not well-controlled. Especially for electropolymerisation, the morphology of the resulting PEDOT is much complicated, since electropolymerised PEDOT is closely defined by the polymerisation conditions including potential, electrolytes, temperature, dopants, monomer side chains and electrochemical techniques ${ }^{102,103}$.

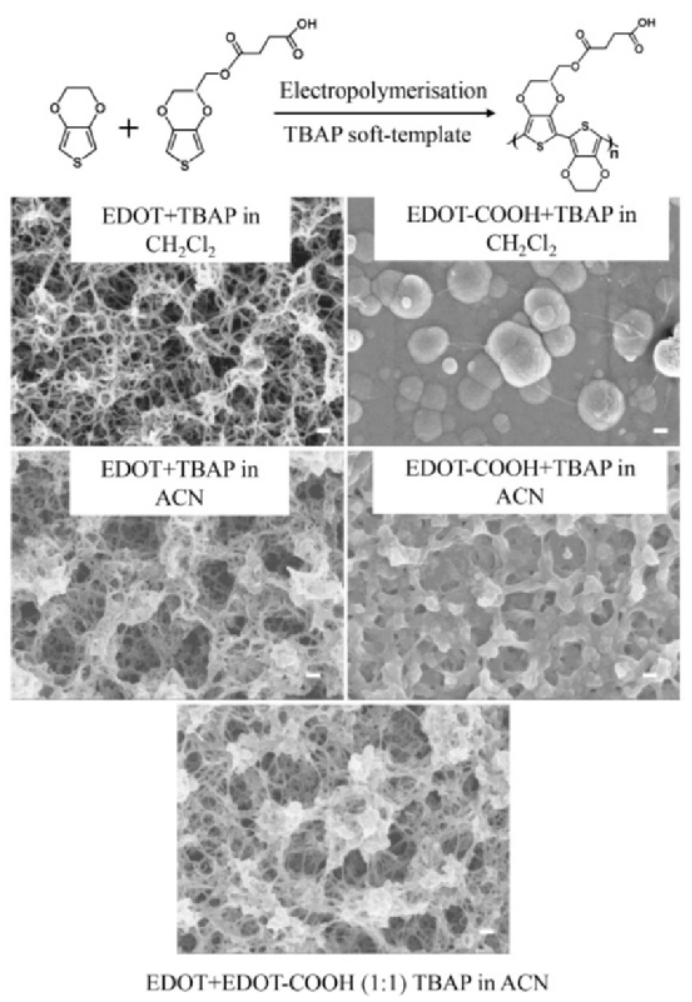

Figure 4.9 Electropolymerisation of EDOT, EDOT-COOH, or their co-polymerisation in the appearance of TBAP in $\mathrm{CH}_{2} \mathrm{Cl}_{2}$ or $\mathrm{ACN}$, the scale bar is $200 \mathrm{~nm}$.

Tetra-butylammonium perchlorate (TBAP) is supposed to contribute to the formation of nanostructured PEDOT with the disassociated perchlorate anions $\left(\mathrm{ClO}_{4}^{-}\right)$doped into PEDOT backbone and $\mathrm{TBA}^{+}$attracted to the lone-pair electrons of the oxygen atoms of EDOT monomer ${ }^{100}$. However, we found that the solvent and monomer side chain also influence the PEDOT structure. As shown in Figure 4.9, PEDOT formed nanofibre type nanostructure when prepared from TBAP in dichloromethane $\left(\mathrm{CH}_{2} \mathrm{Cl}_{2}\right)$. As a comparison, PEDOT bearing a 4-methoxy-4-oxobutanoic acid (PEDOT-COOH) formed compact dense film at the bottom and sub-micro structured PEDOT on the top. When it was prepared in acetonitrile (ACN), PEDOT showed nanofibrous structure with 
narrower diameter size compared that of PEDOT in $\mathrm{CH}_{2} \mathrm{Cl}_{2}$, while the fibrous structure of PEDOT-COOH remained on the top with a closed-structured film. The structural improvement for PEDOT-COOH could be ascribed to the increased polarity of ACN compared to that of $\mathrm{CH}_{2} \mathrm{Cl}_{2}$. To modulate the PEDOT interface with functionality for bioconjugation and nanostructure for electrochemical activity simultaneously, EDOT and EDOT-COOH monomer were co-polymerised into nanofibrous PEDOT-COOH with the assistance of TBAP in ACN, in which the fibre diameter and carboxylic acid density could be controlled through adjusting the ratio between EDOT and EDOT$\mathrm{COOH}$.

In addition to these chemical template directed growth, several physical approaches have been applied in the synthesis of PEDOT structures, involving electrospinning technology for nanofibre or nanotubes ${ }^{104}$ and imprinting for nanoarrays ${ }^{96}$.

\subsubsection{Nanostructured Interface in Sensing and Biosensing}

Bulk PEDOT encounters several limitations in specific applications in chemical sensing and biosensing regarding sensitivity, selectivity, and response time. Nanostructured PEDOT inherits prominent physicochemical properties from its bulk polymer equivalents, while acquiring beneficial characteristics of nanomaterials such as large active surface area to promote catalytic reactions. For instance, in Paper I, hierarchically structured PEDOT CMs electrodes could provide a larger accessible electroactive surface (2.8 times larger than that of the benchmark PEDOT:PSS) and inter-particle space, thus improving electrode kinetics towards the electrooxidation of $\mathrm{NADH}$ at a lower potential and with higher sensitivity and wider linear range compared to the compact structure of conventional PEDOT:PSS electrodes, due to large surface area, interfacial transport, high mass transfer kinetics and shortened diffusion paths, as shown in Figure 4.10.

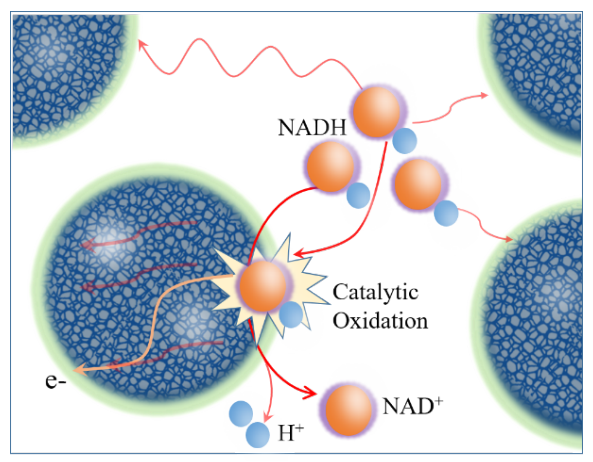

Figure 4.10 Structured PEDOT facilitates the electrochemical catalytic oxidation of NADH. 


\subsection{PEDOT Derivatives}

Owing to its excellent electronic/ionic conductivity and electrochemical redox reversibility, PEDOT shows promising analytical performance as transducers in electrochemical sensing and is capable of responding to a physical stimulus (e.g. pressure and strain $\left.{ }^{105}\right)$, a chemical molecule level (e.g. NADH and DA) or a biological change (e.g. neural record ${ }^{106}$ ). Further exploration of PEDOT as the sensing element and transducer in biosensing, that can specifically recognise biomarkers and subsequently transduce the corresponding bioelectronic information into electronics, is attractive. Two strategies have been used intensively for the incorporation of biorecognition molecules by either doping of biomolecules into PEDOT matrix during the polymerisation process or bioconjugation through functionalised PEDOT with reactive functional groups. However, doping of biomolecules into the PEDOT matrix may disrupt the conductivity and surface properties of PEDOT. To address this issue, PEDOT derivatives bearing functional groups emerge as a promising alternative PEDOT material for anchoring biorecognition molecules under the premise of maintaining the basic characteristics of PEDOT.

\subsubsection{PEDOT Derivative Properties}

Based on the intrinsic organic polymer property of CPs, the rich synthetic chemistry endows PEDOT with versatile functional groups that facilitate effective immobilisation of biomolecules via covalent chemistry for biosensing applications. Various functional groups have been introduced to EDOT monomer as a side chain. Figure 4.11 summarises some of the representative functional EDOT derivates, such as chloromethyl-EDOT (EDOT-Cl), azidomethyl-EDOT (EDOT-N ${ }_{3}$ ), hydroxymethylEDOT (EDOT-OH), carboxy-EDOT (EDOT-COOH) and aminomethyl-EDOT (EDOT-NH $\mathrm{N}_{2}$. These elementary functional groups on the side chain serve as the basis to enable further modification of the PEDOT with other chemical and biochemical functionality. For instance, EDOT-OH is one of the most widely used EDOT derivative. As shown in Figure 4.11, the -OH group enables the nucleophilic substitution reaction for etherification or esterification to expand the functional groups on the side chain ${ }^{107}$.

The introduction of functional groups on the side chain endows EDOT and the resulting PEDOT with new properties. The solubility of EDOT monomer in aqueous solution can be increased when a lyophilic group is introduced as the side chain, which facilitates the polymerisation of EDOT derivative in water without using the surfactant and organic solvent ${ }^{91,108}$. In some cases, the side chain in the EDOT derivative is favourable for the formation of nanostructures with the template-free approach, such as the formation of tube array by using EDOT-OH ${ }^{100}$. In addition, the $\pi$-conjugation and energy band gap of the PEDOT backbone can be tuned by the side chain which results in modulation of 
the optical and electrical properties ${ }^{100,}{ }^{109}$. What is more, the wettability (hydrophobicity/hydrophilicity) of PEDOT derivative surface can be controlled by the long hydrophobic (e.g. fluorinated aliphatic chain) ${ }^{110}$, hydrophilic (e.g. aliphatic sulphonate) ${ }^{111}$ or zwitterionic (phosphorylcholine) $)^{82}$ groups on the side chain, which can be used to reduce nonspecific adsorption, control protein absorption and enhance cell adhesion $^{112-114}$.

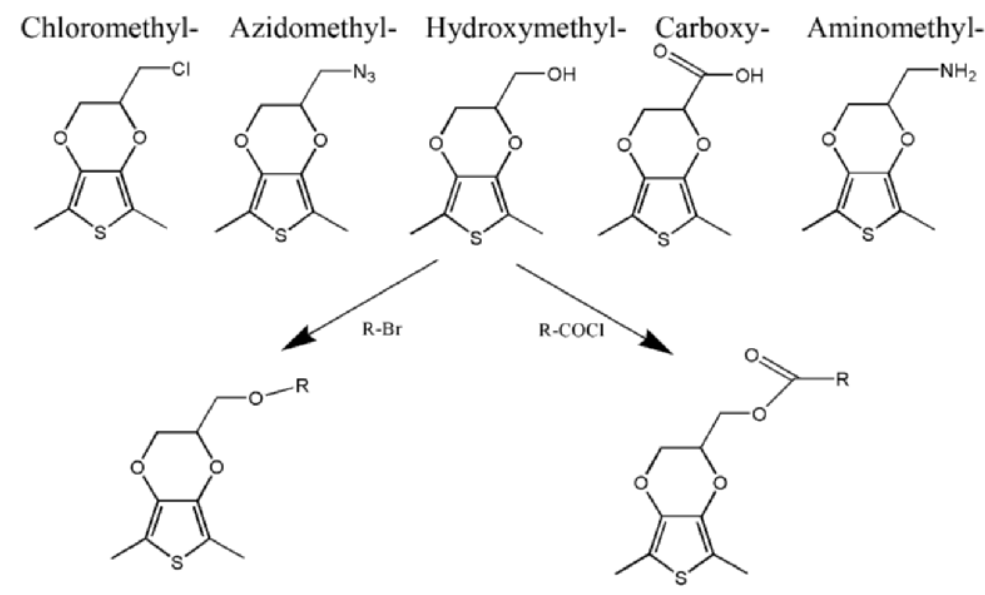

Substitution

$\mathrm{R}=$
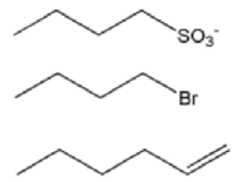

Acyl substitution/Esterification

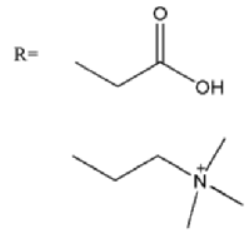

Figure 4.11 Representative EDOT derivatives and post-modification of EDOT-OH.

PEDOT derivatives provide anchoring sites for biorecognition molecules through noncovalent or covalent approaches and can then be used for biosensing. The density of the functional groups on the electrode surface for immobilisation can be controlled precisely by adjusting the ratio of different EDOT and EDOT derivative monomer. For instance, Goda et al. ${ }^{82}$ developed a new EDOT derivative bearing a zwitterionic phosphorylcholine group for the specific detection of an acute-phase biomarker, human C-reactive protein (CRP), by DPV in the appearance of ferricyanide/ferrocyanide probes. Hai et $a l .{ }^{84}$ synthesised a new EDOT derivate bearing an oxylamine group (EDOTOA) that could be electrochemically copolymerised with EDOT in different ratios. The addition of sialyllactose to the side chain of PEDOT through oxylamine group realised 
label-free detection of human influenza A virus (H1N1) by potentiometry with high sensitivity.

\subsubsection{PEDOT-COOH}

PEDOT bearing carboxylic acid groups is an important PEDOT derivate that delivers profound value for sensing and biosensing applications because of: 1) improved aqueous solubility of EDOT-COOH ${ }^{91}$; 2) the negative-charged effect ${ }^{114}$; 3) high biocompatibility ${ }^{15}$; 4) capability for bioconjugation with a large variety of biological molecules, such as nucleotides and proteins (antibodies and enzymes). As shown in Figure 4.12, carboxylic acid group containing side chains are introduced through nucleophilic substitution reactions towards the hydroxyl group in EDOT-OH by etherification with methyl bromoacetate (routine $1, \mathrm{C}_{2}$-PEDOT-COOH) or esterification with succinic anhydride (routine 2, $\mathrm{C}_{4}$-PEDOT-COOH) ${ }^{116,117}$.

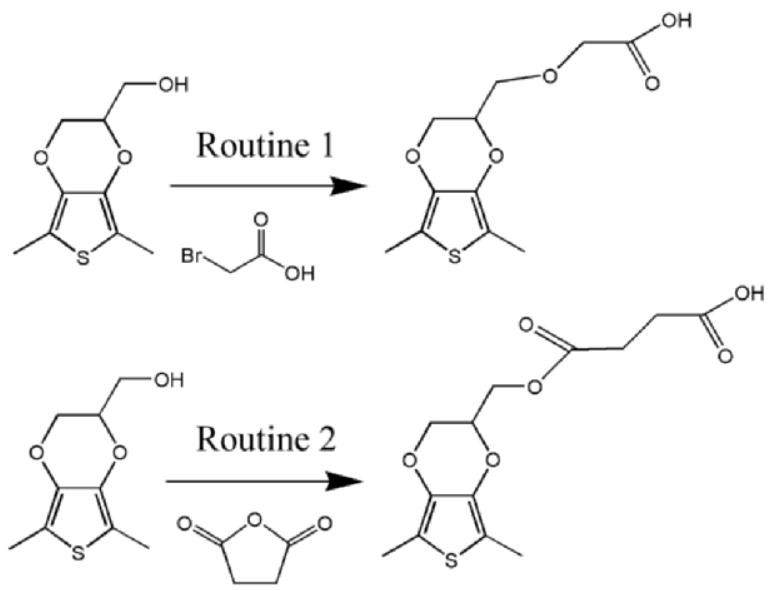

Figure 4.12 Two routines for synthesis of EDOT-COOH from EDOT-OH.

\subsubsection{Biofunctionalisation of PEDOT-COOH}

The biofunctionalisation of PEDOT-COOH with biological molecules can be achieved by using ethyl(dimethylaminopropyl) carbodiimide (EDC)/N-hydroxysuccinimide (NHS) chemistry. As shown in Figure 4.13, the carboxylic acid group is activated by EDC via the formation of an amine-reactive $\mathrm{O}$-acylisourea intermediate that is not stable in aqueous solution, and then the addition of NHS stabilise amine-reactive intermediate by converting it into an amine-reactive succinimide ester. The subsequently added amine containing biomolecules onto the electrode surface reacts spontaneously with such amine reactive ester to covalently link the biomolecules to the electrode surface. Finally, the excess active ester is de-activated by ethanolamine. 


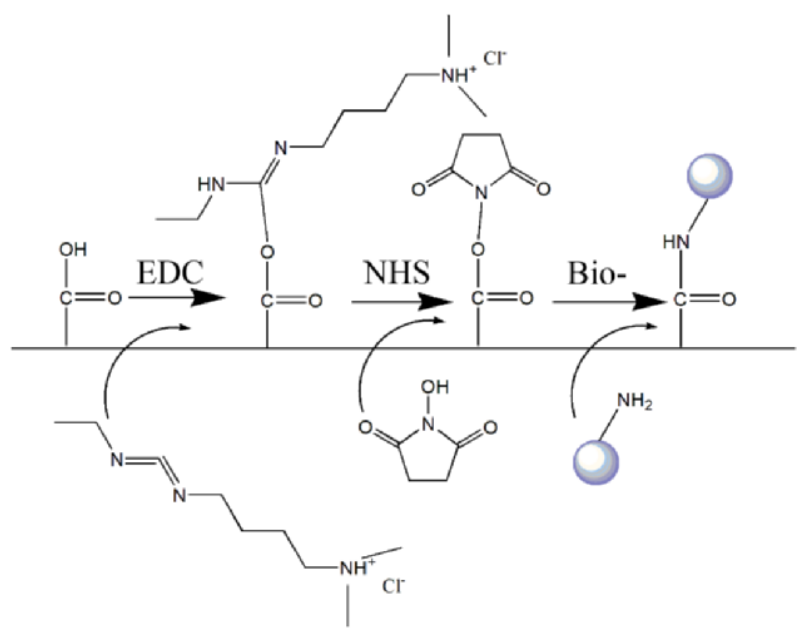

Figure 4.13 Amine containing biomolecule covalent coupling to carboxylic acid on the electrode surface through EDC/NHS chemistry.

Successful biofunctionalisation of PEDOT-COOH with biorecognition elements (e.g. antibody and nucleic acid) via EDH/NHS have been developed for affinity biosensing. For instance, Sekine et al. ${ }^{115}$ demonstrated the electropolymerisation of PEDOT-COOH nanodots on ITO glass, and then streptavidin was conjugated onto PEDOT-COOH via EDC/NHS chemistry, followed by the affinity attachment of biotinylated epithelial cellular adhesion molecule antibody (anti-EpCAM) to direct these antibodies onto the surfaces. The biofunctionalised PEDOT-COOH with antibody was successfully used for the capture of MCF7 cancer cell with over expressed EpCAM on the cell surface. Similarly, Luo et al. ${ }^{118}$ reported the co-polymerisation of EDOT-OH and EDOT-COOH with controlled surface density of carboxylic groups for immobilisation of oligonucleotide probes for monitoring of DNA hybridisation efficiency.

In addition to the biofunctionalisation of PEDOT for affinity biosensing, the bioconjugation of enzyme molecules onto PEDOT surface can be used for catalytic biosensing of a large variety of metabolites, realising a relay bioelectrochemical signal transduction. To achieve a PEDOT-based biointerface with high analytical performance for a catalytic biosensor, both the immobilisation of the enzyme on PEDOT as sensing element for specific recognition of analytes and the subsequent signal transduction at the PEDOT interface as transducer element are important. Therefore, in Paper IV, we aimed to prepare a functional PEDOT interface with a tunable 3D nanofibrous network and carboxylic acid groups (i.e. Nano-PEDOT-COOH) via controlled copolymerisation of EDOT and EDOT-COOH monomers. The carboxylic acid groups provide an anchoring site for the stable immobilisation of an NADH-dependent 
dehydrogenase (i.e. LDH) via EDC/NHS chemistry, for the fabrication of a Bio-NanoPEDOT-based biosensor for lactate detection (Figure 4.14), realising a relay catalytic bioelectrochemical signal transduction mechanism, during which LDH catalyses the conversion of lactate to pyruvate and $\mathrm{NADH}$ in the presence of $\mathrm{NAD}^{+}$, followed by the Nano-PEDOT electrode catalytically oxidising NADH to generate a signal current.

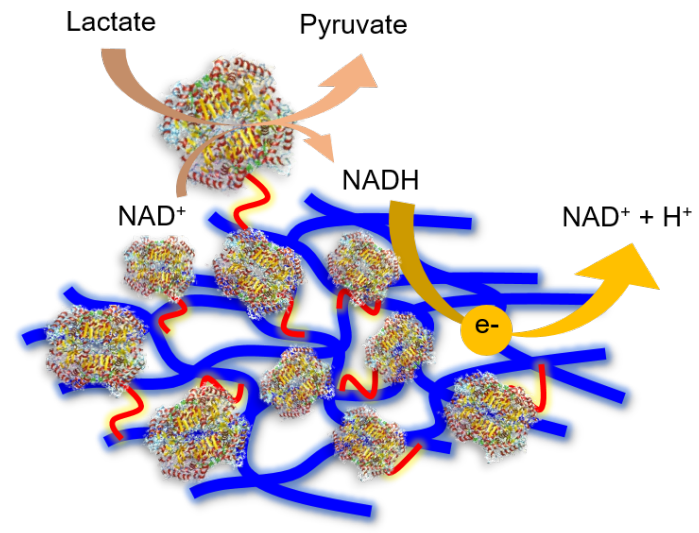

Figure 4.14 Co-polymerisation of EDOT and EDOT-COOH into a nanofibrous PEDOT-COOH network for enzyme coupling for lactate biosensing.

\subsection{PEDOT Composites}

\subsubsection{Pristine PEDOT}

Due to its fascinating electronic and ionic conductivity in a doped state, reversibility, and its unique mechanical properties, PEDOT, as one of the most widely researched CPs, has been extensively used for electrode modification for a wide range of applications including energy conversion and storage such as organic photovoltaic cells $^{119}$ and supercapacitors ${ }^{120}$, antistatic coatings ${ }^{121}$, organic light emitting diodes ${ }^{122}$, electrochromic devices ${ }^{123}$, organic filed effect transistors ${ }^{124}$, analytical and biomedical applications such as sensors and biosensors ${ }^{125}$, actuators ${ }^{126}$, tissue engineering ${ }^{127}$ and drug release $\mathrm{e}^{104}$ etc. In the field of sensors and biosensors, PEDOT is a promising candidate as an electronic transducer for the determination of physical, chemical and biological parameters. The electronic transduction of analytes/mediators/intermediates at PEDOT interface relies on the electron transfer at the PEDOT interface and the redox state (doping level) of PEDOT ${ }^{128}$. Despite the advantageous morphological and structural properties of PEDOT, the requirement for well electrochemical performance with respect to high sensitivity, fast response, low LoD and good selectivity from a 
pristine PEDOT modified electrode remains unfulfilled for a variety of analytes, especially for catalytic sensors and biosensors.

\subsubsection{PEDOT Composites in Sensing and Biosensing}

PEDOT composites refer to the PEDOT complex associated with one or more other functional components. To improve the performance of PEDOT for electrochemical signal transduction in sensing and biosensing, various functional materials with unique physical-chemical properties, have been combined with PEDOT, such as carbon-based nanomaterials, metal/metal oxide, dyes and biomolecules etc. Figure 4.15 shows some representative nanomaterials incorporated for PEDOT composites. Such materials are usually incorporated into the PEDOT matrix either as negatively-charged dopants by virtue of positively-charged backbone of PEDOT during the polymerisation process, or as additives blended with processable PEDOT. The incorporation of these nanomaterials endows PEDOT with enhanced or even extended electrochemical properties with an increased active surface area, enhanced charge/electron transfer, high catalytic activity and improved mechanical stability, and thus high sensitivity, fast response, low LoD and good selectivity. In the meantime, PEDOT also facilitates the dispersion and anchoring of these nanomaterials on the modified electrode surface with minimised aggregation, stacking and leakage.

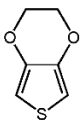

Incorporation as dopants

Carbon materials
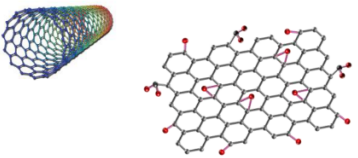

Dye

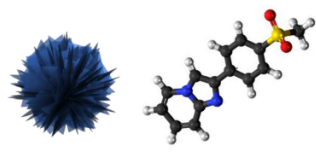

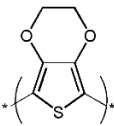

Post-blending

Metal/Metal oxide
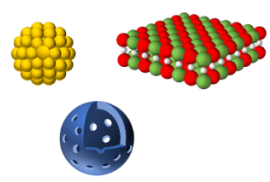

Biomolecules

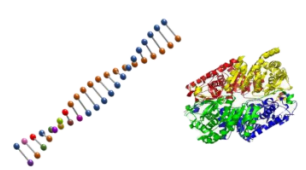

Figure 4.15 Incorporation of functional materials into PEDOT as dopants via doping or additives via post-blending. 
Carbon-based nanomaterials of different dimensions, such as 0D carbon quantum dots, 1D CNTs, 2D graphene, and 3D carbon network, have been used for the development of electrochemical sensors and biosensors due to their excellent conductivity, high chemical stability and large surface area. These carbon-based nanomaterials can serve as supporting templates to direct the formation of PEDOT nanostructures with high surface area, while PEDOT can improve the dispersion of carbon materials with minimised $\pi$ - $\pi$ stacking. Besides, metals and metal oxides are another class of functional materials for PEDOT composites, such as $\mathrm{Au}, \mathrm{Pt}, \mathrm{Pd}, \mathrm{ZnO}, \mathrm{Fe}_{3} \mathrm{O}_{4}$ in the form of nanoparticles, nanowires, nanoclusters, nanoflowers etc. These provide unique electrochemical catalytic activity towards chemical analytes and thus contribute to the improved analytical performance with low overpotential and high sensitivity. Additionally, some dyes or pigments with redox reversibility provide PEDOT with better signal transduction by their catalytic effect towards the enzymatic products, such as Prussian blue and Meldola's blue. What is more, biological active materials can also be doped or entrapped into PEDOT matrix for improved biocatalytic activity or selectivity, including small biomolecules (e.g. hemin) and biomacromolecules (e.g. enzymes, antibodies, nucleic acids). These PEDOT composites synergistically enhance electrochemical sensing and biosensing ability for a large variety of target chemical and biochemical analytes, such as acetaminophen ${ }^{129}$, dopamine ${ }^{130}, \mathrm{NADH}^{131}, \mathrm{H}_{2} \mathrm{O}_{2}{ }^{132}$, glucose $^{133}$ and lactate ${ }^{134}$ etc.

Among these functional nanomaterials mentioned above, 2D graphene and its derivatives including graphene oxide $(\mathrm{GO})$ and its reduced form (reduced graphene oxide, RGO) are attractive to serve as dopants for PEDOT due to their high specific surface area, unique physicochemical property and compatibility. For GO, the abundant oxygen-containing functional groups, including hydroxyl $(-\mathrm{OH})$, carboxyl $(-\mathrm{COOH})$ and carbonyl $(\mathrm{C}=\mathrm{O})$ endow $\mathrm{GO}$ with negatively-charged surface, which facilitates the high dispersibility of GO in aqueous solution with long-term stability ${ }^{135}$, as well as the efficient doping into the PEDOT positively-charged backbone ${ }^{136,137}$. Nevertheless, the PEDOT:GO modified electrode suffers from the low conductivity and thus sluggish electron transfer kinetics for electrochemical performance owing to the well-known electronic insulating property of GO. Therefore, the reduced form of GO by chemical/electrochemical reduction, i.e. $\mathrm{RGO}$, restores the conductivity and electrochemical catalytic activity, and is used alternatively as dopant for PEDOT. However, the deprived negatively-charged oxygen-containing groups on RGO surfaces are inclined to agglomerate by $\pi-\pi$ stacking, resulting in low aqueous dispersibility and stability. They affect the doping efficiency of RGO into PEDOT and result in poor homogeneity of the resulting PEDOT:RGO composite film. 
In Paper III, we developed a bi-functional sulphonate-coupled reduced graphene oxide (S-RGO) with both high reduction degree and negatively-charged sulphonate terminal functional groups severing as an efficient dopant for PEDOT. The resulting S-RGO combined the advantages of $\mathrm{GO}$ and $\mathrm{RGO}$ mentioned above with good aqueous dispersibility, high conductivity and negatively-charged sulphonate terminal functionality (Figure 4.16), which is favourable for electropolymerisation of EDOT and effective doping to synthesise PEDOT:S-RGO with good homogeneity. The final PEDOT:S-RGO modified electrode not only possesses lower equivalent series resistance and charge transfer resistance, but showed enhanced electrochemical kinetics towards $\mathrm{Fe}(\mathrm{CN}) 6^{3-44-}$, DA, NADH and $\mathrm{H}_{2} \mathrm{O}_{2}$ catalytic transduction in the manner of faster electron transfer (smaller $\triangle \mathrm{Ep}$ ), increased current response and lower peak potential than that of PEDOT:GO and PEDOT:RGO.

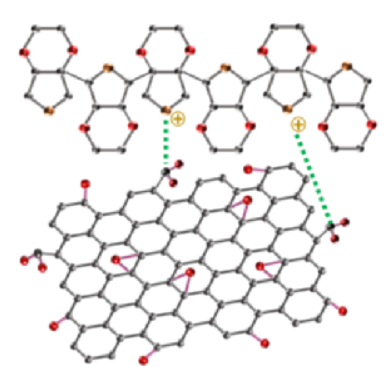

PEDOT:GO

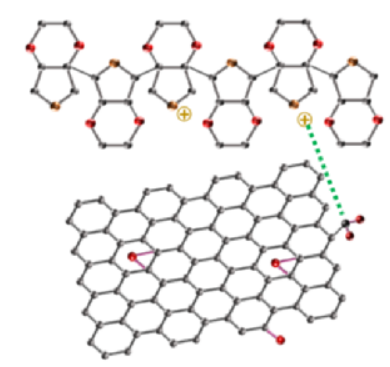

PEDOT:RGO

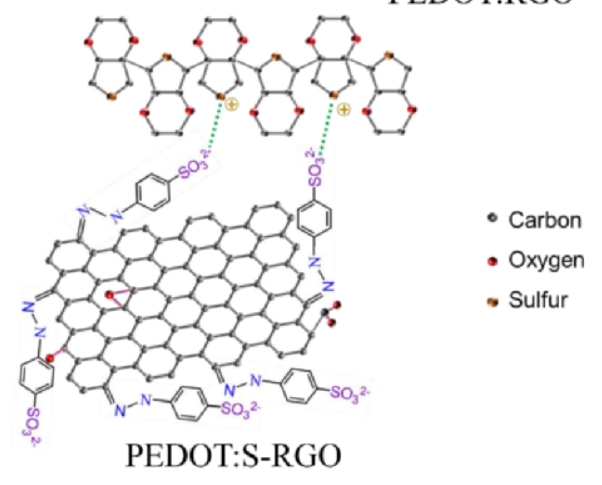

Figure 4.16 PEDOT doped with graphene derivative GO, RGO and S-RGO. 


\section{Chapter 5 Polymer-based Flexible and Wearable Bioelectronics}

\subsection{Flexible and Wearable Bioelectronics}

Nowadays, the ever-growing demand for healthcare services or self-health monitoring propels the development of $\mathrm{PoC}$ devices to track an individual's physiological information in decentralised locations at a physical fitness level or for medical treatment. The advancement in flexible and wearable bioelectronics in recent years is driving the trend of changing from hand-held devices, that dominate the market currently, to the next generation of flexible and wearable sensing devices that can be intimately attached to human skin and tissue externally or implanted internally, to deliver accurate, continuous and remote measurement of physiological parameters or biomarkers.

Flexible and wearable sensing devices possess several intriguing advantages for personal healthcare management. Their light-weight, flexible or conformable properties are important to fulfil the requirements of mechanical behaviour for intimate interfacing with soft, deformable, and complex biological tissue internally with enhanced physical/biological event recognition and transduction ${ }^{138}$. The attachment of such device externally onto human skin provides the promising potential for non-invasive or lessinvasive continuous monitoring. And the flexibility, stretchability, durability and userfriendly simplicity of the assembled sensors are crucial for long-term wearing without conspicuous discomfort.

The general structure of flexible and wearable bioelectronic devices is composed of several functional components, including flexible and compatible substrates, power supply, sensing elements for signal recognition and transduction, electrical circuits and data processing systems. A supporting substrate is preferably flexible, stretchable and highly biocompatible compared to the conventional rigid and bulk substrate materials, such as fabrics, organic polymers and silicone elastomers. Continuous power supply is vitally important for the reliable and continuous function of wearable or implantable bioelectronic devices. Rechargeable batteries (e.g. lithium-batteries) are very commonly used for portable electronic devices. Apart from batteries, various potential strategies for battery-free power sources and power storage have been developed in recent years on flexible substrates, such as printed solar cells, supercapacitors, piezoelectricity generators, triboelectric generators, thermoelectricity generators, bio-fuel cells and wireless power transfer ${ }^{139}$. Sensing elements and electrical circuits can be realised by 
conductive metal, metal oxide, carbon and CPs with advanced processing techniques (lithography, vapour deposition, printing etc.) and their modification or functionalisation. Data processing system and wireless communication systems are integrated onboard to process, analyse and deliver data ${ }^{140}$.

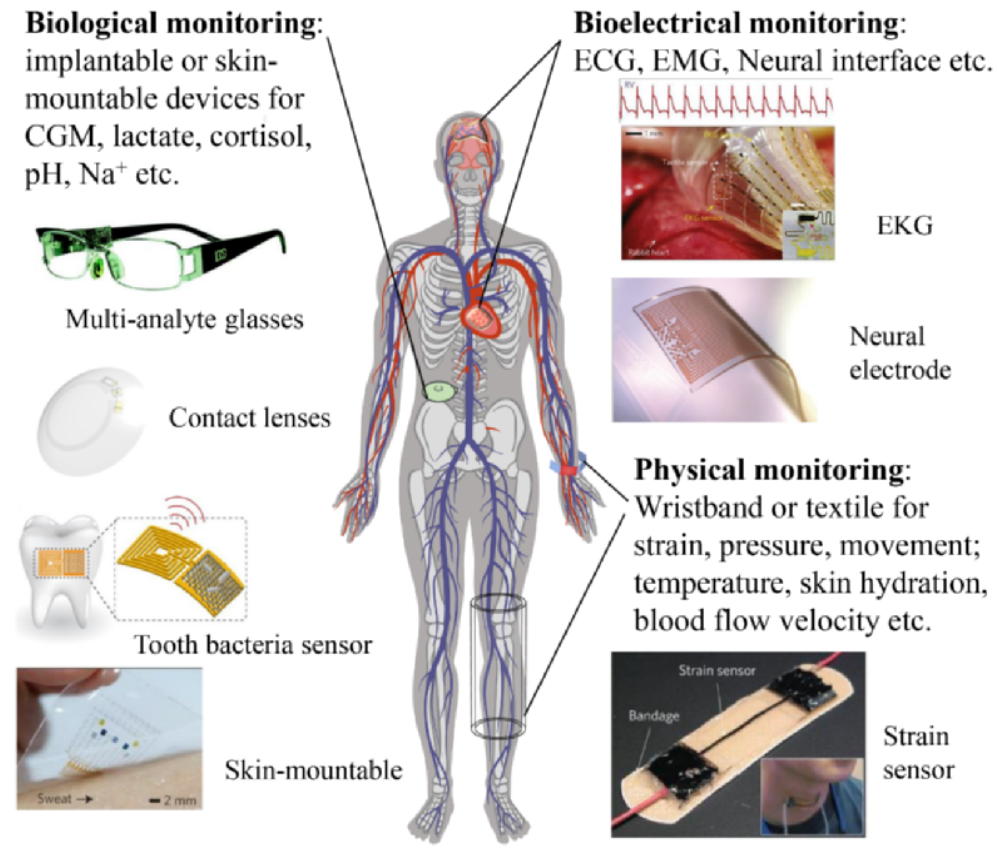

Figure 5.1 Flexible and wearable bioelectronic devices for healthcare monitoring. Parts of the insets are adapted with permission from Sempionatto, J.R., et al. ${ }^{141}$ for multianalytic glasses, Park, J. et al. ${ }^{142}$ for contact lenses, Mannoor, M.S., et al. ${ }^{143}$ for tooth bacteria sensor platform, Lee, H. et al. ${ }^{144}$ for skin-mountable platform, Kim, D.H., et $a l .{ }^{145}$ for electrogram (EKG), Tybrandt, k., et al. ${ }^{146}$ for neural electrode, Yamada, T. et $a l .{ }^{147}$ for stain sensor platform.

Based on these unique properties, the advancement in the field of flexible and wearable bioelectronics can be used for the development of wearable or implantable sensors and biosensors to detect relevant physical and biochemical signals for healthcare monitoring and disease diagnosis as shown in Figure 5.1. Firstly, flexible and wearable bioelectronic devices are promising to be used as human-machine interfaces with intimate contact with human tissues for bioelectricity monitoring, such as ECG, electromyogram (EMG), electrooculogram (EOG), electroencephalogram (EEG) of the central nervous system ${ }^{148}$. On the other hand, a variety of physical signs can be monitored by flexible and wearable electronic devices in the form of wristband or 
textiles, including strain, pressure, body movement, temperature, sweat rate and blood flow velocity etc. Additionally, through measurement of biofluids including blood, sweat, saliva, tear by implantable or skin-mountable bioelectronic devices, physiological information from a variety of biomarkers (e.g. glucose, lactate, cortisol etc.) can be collected in real-time. What is more, flexible and wearable bioelectronic devices hold great promise for the integration of multiplexed monitoring systems to detect multiplex biomarkers ${ }^{14}$ and feedback therapy system, such as the insulin infusion $^{149}$.

\subsection{Soft and Flexible Materials}

Advances in the field of soft and flexible materials have provided an unique opportunity to the development of flexible and wearable bioelectronic devices with minimised mismatch between conventional rigid electronic devices and soft tissues in nature. A key feature for a flexible and wearable bioelectronic device is to develop an effective interface possessing both mechanical flexibility and electronic properties. In this section, a variety of soft and flexible materials are discussed briefly regarding to their functions for the fabrication of effective human-machine sensing interface as base substrates and microfluidics, as well as conducive contacts, tracks and signal sensing interfaces.

Substrates, severing as the foundation of the electronic device, play an important role to support all the active components. Mechanical flexibility is the primary characteristic for substrates, ensuring the intimate contact between the electronic device interface with human tissue enabled by their bendable and conformable shaping into different structures. The ability to synthetically tailor organic polymers offers diversity and versatility over a wide range of flexible substrates, ranging from synthetic polymers such as polyethylene terephthalate (PET), polyurethane (PU), poly(methylmethacrylate) (PMMA), polycarbonate (PC), polyimide (PI) etc., to natural polymers and biodegradable polymers including cellulose, hydrogel and silk etc ${ }^{150}$. Besides, membranes and paper are also attractive and inexpensive materials for using as substrate in lateral-flow membrane-based strip tests and microfluidic paper-based analytical devices ( $\mu$ PADs). What is more, textiles and fabrics from wearable objects in daily life provide a large range of ideal materials as base substrates for flexible and wearable devices, due to their daily routine utilisation and good mechanical stability. In some cases, the substrates should possess high stretchability or elasticity to match the Young's modulus of human tissues, which is usually achieved by either the usage of elastic organic/inorganic polymers such as polydimethylsiloxane (PDMS) or the special design of the architectures in waved networks. Compatibility is of vital importance for 
biomedical devices to minimise the immune responses, especially for implantable bioelectronics devices.

To realise continuous and accurate analytical operations, microfluidics can be built on the flexible and wearable bioelectronic devices to provide additional function to extract and capture sample at the device-tissue interface with minimised sample evaporation and external contamination ${ }^{151}$. For instance, in a skin-mountable device, an epidermal microfluidic system can collect the sweat from the skin surface and then transport biofluid sweat through the microchannels into separated microchambers for multianalysis including sweat volume, sweat rate, creatinine level, $\mathrm{pH}$ and glucose level etc ${ }^{152,153}$. Conformable PDMS is widely used for the fabrication of microfluidic systems via soft-lithography methods or laser cutting ${ }^{154}$.

The conversion of physiological events into electric signals is usually realised by the recognition and transduction of these parameters into variations of the sensing layers' electrical parameters, such as resistance, conductivity, current flow and potential change. Such conversion relies on the fabrication of conductive contacts, tracks and sensing interface on the flexible substrate that can conform along with the base substrate. Conventionally, rigid conductors (e.g. metal) and semi-conductors (e.g. silicon) are the most commonly used materials for electronic devices. However, their stiffness and brittleness are not ideal for flexible and wearable bioelectronic devices. Much effort, therefore, has been devoted to improve the flexibility of these inorganic electronic materials by reducing their thickness and by tailoring their architectures with wavy configuration, island-bridge structure and serpentine or fractal interconnects design $^{148}$. Metals, such as $\mathrm{Au}, \mathrm{Pt}, \mathrm{Cu}$ and $\mathrm{Ag}$, and metal oxides, such as ITO and indiumzinc-oxide (IZO) can be deposited on a substrate surface in the form of thin films with adequate flexibility by electroplating, sputtering and vapour deposition. Alternatively, these metals can be prepared in the form of nanowires or nanotubes, and then used for the fabrication of metal mesh on a stretchable substrate with interconnected structure and corresponding stretchability ${ }^{150}$. Carbon-based materials are another class of inorganic materials used for the fabrication of flexible electrodes, including CNTs, graphene, graphite, and carbon black, which are common in the form of ink that allows the deposition on the flexible substrates via different printing techniques. Compared to the inorganic materials, CPs possess several advantages to be used as biotic/abiotic interface owing to their high electrical/ionic conductivity, intrinsic mechanical flexibility, biocompatibility, chemical structure and morphology tunability, light-weight and similarities in polymeric structure with biomolecules. The CPs discussed in Chapter 4 can be deposited on the flexible substrate via printing or coating techniques, in which processable PEDOT:PSS is widely used CPs for organic bioelectronics. 
In the Review paper, we tracked the evolution of affinity sensors from conventional lateral-flow test strips to wearable/implantable devices enabled by soft and flexible materials, including the membrane and paper materials which have been so successfully applied in PoC tests, multifarious polymer designs that provide both the base materials for sensor design and more advanced functionalised materials that are capable of both recognition and transduction, as well as skin-mountable, textile materials-based and contact lens-based materials for flexible and wearable sensing.

In Paper V, we developed a flexible and wearable sensor platform based on PI. As shown in Figure 5.2, PI served as the base substrate with high flexibility and durability owing to its polymeric property. Graphene network was mask-freely patterned on PI by laser (i.e. laser induced electrode, LIG) with 3D honeycomb-structure serving as conductive contacts, tracks and sensing interface, and subsequent deposition of PEDOT reinforced the mechanical stability as conductive polymeric binder. Such an LIG electrode was successfully integrated into a $3 \mathrm{D}$ waistband for a flexible and wearable lactate biosensor.

a. Design and preparation of flexible LIC electrodes

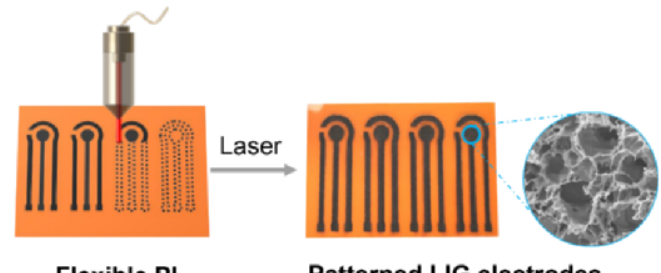

Flexible PI

Patterned LIG electrodes

\section{b. Integration of flexible and wearable lactate biosensor}
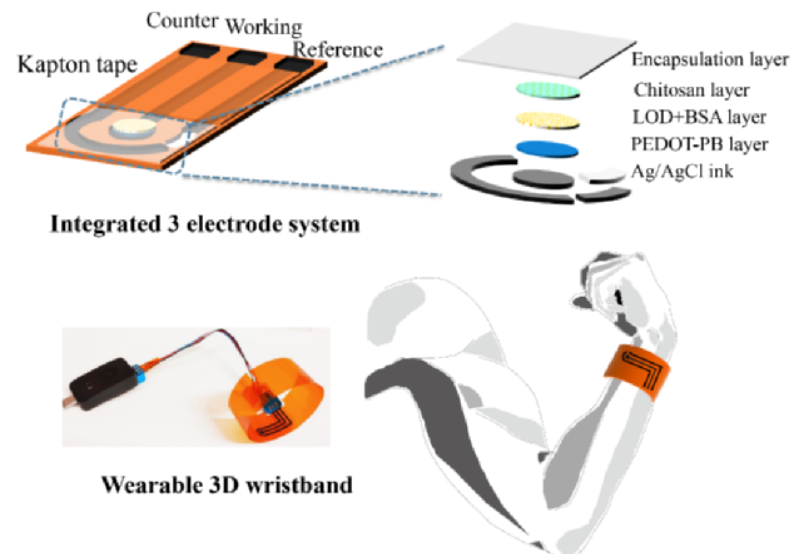

Figure 5.2 Schematic diagram of patternable LIG electrode system in a flexible and wearable lactate biosensor. 


\subsection{All-polymer based Bioelectronics}

Nowadays, metallic, inorganic semiconductors and carbon allotropes are still dominating the fabrication of bioelectronic devices by virtue of their rigidity and mechanical stability. However, the development of a new generation of flexible, wearable and implantable bioelectronic devices (i.e. sensors and biosensors) requires the introduction of unique mechanical flexibility and high biocompatibility. Advancements in the synthetic polymer field have facilitated the use of insulating organic polymers as flexible and stretchable substrates to carry all the active components to minimise the mismatch with soft human tissue ${ }^{155}$. Apart from the use of polymers as substrates, further exploration of CPs as electrochemical active components has been made recently. The combination of conventional polymer as the substrate and advanced CPs as active components results in a new concept of "all-polymer" based electronics and bioelectronics for biosensing and biomedical applications.

The organic electrochemical transistor (OECT) is a unique device using CPs (e.g. PEDOT:PSS) as organic semiconductors for ion-to-electron conversion that can be used for sensing and biosensing with the features of intrinsic signal amplification, low-cost and ease-of-miniaturisation. For instance, Gualandi et al. ${ }^{156}$ exploited an all PEDOT:PSS OECT device with optimised geometries and thicknesses for amperometric signal amplification of faradaic current originating from the electrooxidation of ascorbic acid. Further fabrication of OECT components on the polymeric substrate into all-polymer OECT offer intimate interfacing between solidstate electronics and the biological moiety. Basirico et al. ${ }^{157}$ reported the fabrication of all PEDOT:PSS based OECT on a PET substrate that was reproducible and robust enough for biosensors and low-cost circuitry.

Apart from OECT, all-polymer electrochemical biosensors have been extensively reported in the form of $\mathrm{CP}$ microarrays, microchannels, interdigitated electrodes, printed working electrodes and electrode pairs on substrates including PMMA, paper, Topas ${ }^{\circledR}$ polymer and PDMS for electrochemical detection of virus infection of human cells $\mathrm{s}^{158}$, capture of cardiac and neuronal signals ${ }^{159}$, label-free sensing of antibiotics ${ }^{160}$, detection of influenza A virus ${ }^{161}$ and glucose biosensors ${ }^{162}$. Some important prerequisites of allpolymer based bioelectronics for biosensors is the establishment of a polymer-based bio-interface with a stably immobilised biorecognition element and a related polymerbased transducer interface for signal transduction. In Paper IV, we developed a bioorganic (enzyme)/organic polymer (PEDOT) bio-interface based on the 3D nanofibrous and carboxylic acid functionalised PEDOT, which possesses functionality for bioconjugation and good electrochemical properties for signal transduction towards an all-polymer based biosensor. 


\section{Chapter 6 Summary of the Papers Included}

The papers included in this thesis focus on the tailoring of PEDOT interface for electrochemical sensing and biosensing with improved sensitivity, selectivity and stability, which are classified into 1) tailoring the PEDOT transduction interface for chemical sensing via modulating the charge effect, morphology effect and composite effect (Research Paper I-III); 2) tailoring the PEDOT-based bio-interface for biosensing (Research Paper IV); 3) tailoring the PEDOT-based device interface for flexible bioelectronics (Research Paper V and Review Paper I).

Tailoring the PEDOT transduction interface for chemical sensing: Research Paper

\section{I-III}

\section{Research Paper I}

Positively-charged hierarchical PEDOT interface with enhanced electrode kinetics for NADH-based biosensors
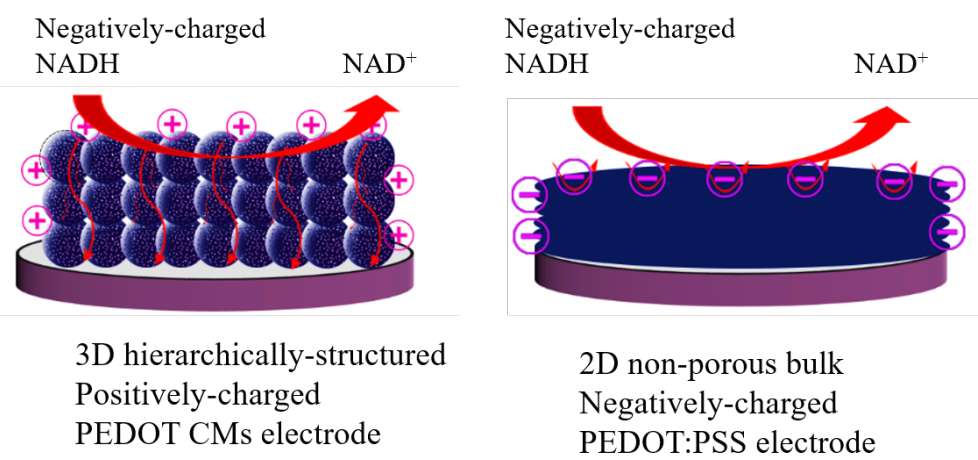

Figure 6.1 Schematic diagram of the PEDOT modified electrodes with charge and structure for NADH detection.

Inherent surface charge (Section 4.2.2) and opened structure (Section 4.3) of the chemically modified electrode are important for the electrode kinetics towards charged analyte molecules facilitated by the high assessable surface area and electrostatic effect.

In this paper, we demonstrated the facile preparation of a positively-charged and hierarchical micro-structured PEDOT electrochemical interface using processable PEDOT colloidal microparticles (PEDOT CMs) as building blocks, which were 
synthesised by hard-template assisted polymerisation. Compared to the bench-mark PEDOT:PSS resulted in non-porous bulk film, PEDOT CMs assembled 3D hierarchical structure possess a 2.8 times larger accessible electroactive surface area with pores and inter-particle spaces. The intrinsic positive charge of the PEDOT CMs further facilitated the electrochemical kinetics of negatively-charged molecules through electrostatic accumulation effect, while PEDOT:PSS showed electrostatic repulsion effect (Figure 6.1).

Based on the synergistic effect of hierarchical structure and positive charge, the PEDOT CMs electrodes exhibited improved electrooxidation of NADH at lower potential and enhanced electrocatalytic activity compared to that of PEDOT:PSS electrodes. The detection of NADH at the PEDOT CMs electrodes realised the wide linear range over $20-240 \mu \mathrm{M}$, with a high sensitivity of $0.0156 \mu \mathrm{A} / \mu \mathrm{M}$ and a LoD of $5.3 \mu \mathrm{M}$. What is more, the PEDOT CMs electrode possesses good anti-interference with large oxidation peak separation from the interferent ascorbic acid, and improved stability.

\section{Research Paper II}

Modulating electrode kinetics for discrimination of dopamine by a PEDOT:COOH interface doped with negatively charged tricarboxylate
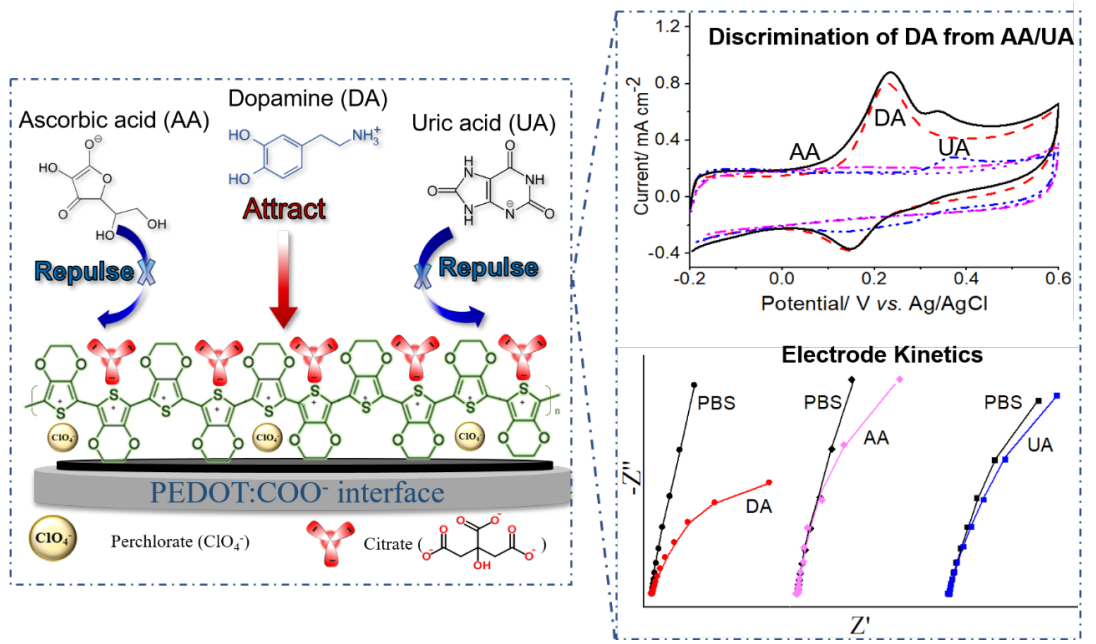

Figure 6.2 Illustration of PEDOT:COOH interface doped with negatively charged tricarboxylate for discrimination of DA by electrostatic effect, and the corresponding improved electrode kinetics for DA at PEDOT: $\mathrm{COOH}$ interface in the appearance of $\mathrm{UA}$ and AA. 
Apart from the positively-charged and hierarchically-structured PEDOT, a negativelycharged and structured PEDOT can be realised simultaneously by the incorporation of dopants as explained in Section 4.2.2.

In this paper, we demonstrated a facile and economic approach for the fabrication of a high-density and negatively-charged carboxylic-acid-group-functionalised PEDOT (PEDOT:COOH) interface using an inexpensive ternary carboxylic acid, citrate, as the dopant. The polymerisation efficiency of PEDOT:COOH was significantly improved with the assistance of $\mathrm{ClO}_{4}{ }^{-}$, yielding a dense PEDOT:COOH film with a high surface density of carboxylic acid groups $\left(0.129 \mu \mathrm{mol} \mathrm{cm}^{-2}\right)$ and homogeneously distributed globular structure.

The CV response of the PEDOT:COOH interface towards positively-charged DA demonstrated a significantly reduced $\Delta E p$ of $90 \mathrm{mV}$ and a 3 -fold increase in the $I_{p a}$ value compared to that of non-functionalised PEDOT interface. Besides, the electrode kinetics of DA, in the presence of the interferents AA and UA at the PEDOT:COOH interface, were further evaluated by CV and EIS, showing increased electrode kinetics and highly selective discrimination of DA. The improvement can be ascribed to that the negativelycharged carboxylic acid groups facilitate the transfer, preconcentration, and permeation of positively charged DA to deliver improved sensing performance while repelling the negatively charged AA and UA interferents. The PEDOT:COOH electrode detected DA over the range of $1-85 \mu \mathrm{M}$, with a high sensitivity of $0.228 \mu \mathrm{A} \mu \mathrm{M}^{-1}$, which is 4.1 times higher than that of a non-functionalised PEDOT electrode $\left(0.055 \mu \mathrm{A}^{-1}\right)$.

\section{Research Paper III}

Bi-functional sulphonate-coupled reduced graphene oxide as an efficient dopant for a conducting polymer with enhanced electrochemical performance

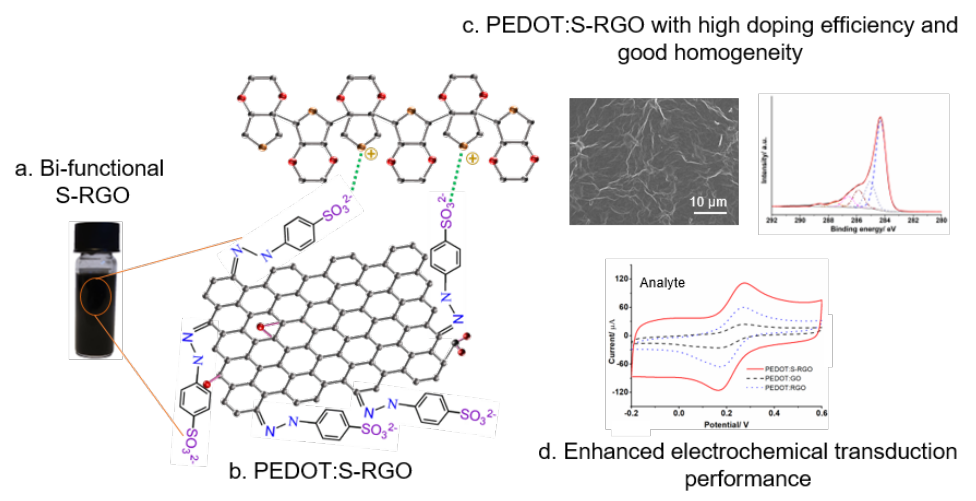

Figure 6.3 Bi-functional S-RGO as dopant for PEDOT polymerisation with enhanced electrochemical transduction performance. 
Despite the easy formation of a tenacious film on the electrode surface via electrochemical polymerisation as $\mathrm{CME}$ for electrochemical application including sensing and biosensing, pure PEDOT-modified electrodes still have the limitation of unsatisfactory catalytic properties towards a variety of analytes and low active surface area for effective electrochemical transduction, which result in low sensitivity, poor selectivity and surface fouling for electrochemical sensing. As discussed in Section 4.5, various functional materials with unique physicochemical properties, usually in their nanostructured architectures, have been combined with PEDOT, such as carbon-based nanomaterials, metal/metal oxide, dyes and biomolecules etc.

In this paper, taking 2D graphene as the model, we evaluated the influence of the quality and physicochemical nature of the dopants to the polymerisation efficiency of PEDOT. A bi-functional graphene oxide derivative possessing high reduction degree and negatively-charged sulphonate functionality, i.e. sulphonate-coupled reduced graphene oxide (S-RGO), was fabricated through one-pot hydrothermal reaction of GO with 4hydrazinobenzosulphonic acid (4-HBS) as the reductant and sulphonate precursor. Such S-RGO combines the advantages of the conventional GO (functionality) and RGO (high conductivity), displaying high aqueous dispersibility and stability (more than 6 months), high electrical conductivity (1493.0 S m $\mathrm{S}^{-1}$ ) and sulphonate functionality.

Due to these specific properties, S-RGO, serving as 2D dopants, demonstrated improved PEDOT electropolymerisation efficiency than that of GO and RGO. The resulting PEDOT:S-RGO displays as a homogenous film on electrode surface with uniformly distributed S-RGO owing to the sulphonate functionality, and possesses low equivalent series resistance and low charge transfer resistance due to the high electrical conductivity of S-RGO.

As a result of the synergistic effect of S-RGO and PEDOT, the PEDOT:S-RGO showed improved electrochemical performance towards 4 different analytes, including $\mathrm{Fe}(\mathrm{CN})_{6}^{3-/ 4-}$, DA, NADH and $\mathrm{H}_{2} \mathrm{O}_{2}$, with respect to faster electrode kinetics, increased current responses, and lower peak potentials compared to PEDOT:GO and PEDOT:RGO.

This bi-functional S-RGO dopant possessing both sulphonate functionality and high conductivity delivered the efficient polymerisation of an advanced PEDOT composites interface with improved electrochemical performance, which could potentially be employed in electrochemical sensors and biosensors. 


\section{Tailoring PEDOT-based bio-interface for biosensing: Research Paper IV}

\section{Research Paper IV}

Tunable 3D nanofibrous and bio-functionalised PEDOT network explored as a conducting polymer-based biosensor

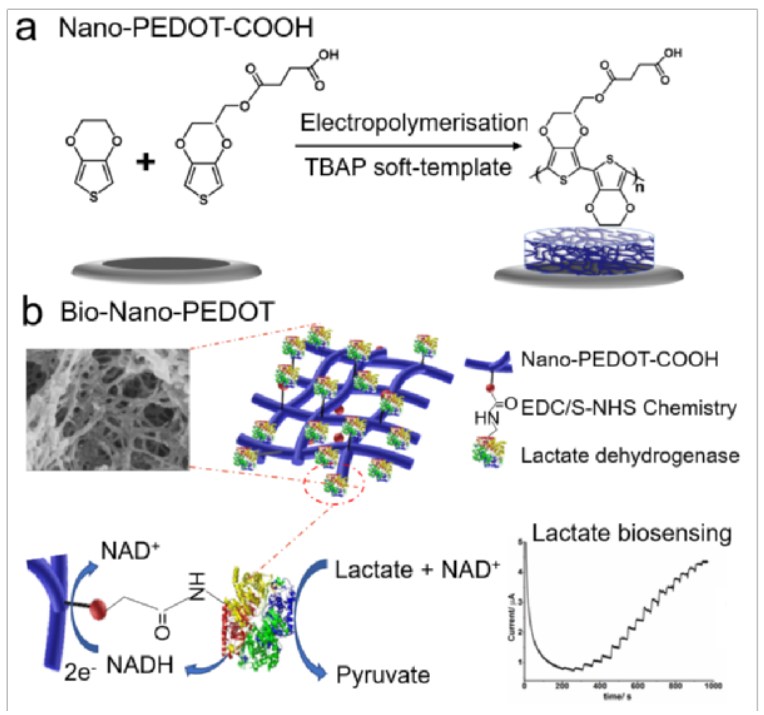

Figure 6.4 Schematic of (a) 3D Nano-PEDOT-COOH network fabrication; (b) NanoPEDOT-COOH for immobilisation of LDH via EDC/S-NHS chemistry for lactate biosensing.

As discussed in Section 2.3 and 4.4.3, PEDOT shows considerable potential as an interfacing material in biosensing to bridge between the organic electronics world and bioelectronics because of its extraordinary redox reversibility, biocompatibility, electrochemical properties and sufficient similarities in its organic chemical nature with biological molecules. In addition, the development of all-polymer biosensors relies on the creation of a CP-based bio-interface with good electrochemical properties and nanostructured morphology for signal transduction that does not need traditional nanocatalysts such as carbon materials, metal, metal oxides or dyes, as well as providing functionality for the bioconjugation of biomolecules.

In this paper, we demonstrated the development of an innovative bioorganic/organic polymer bio-interface possessing functionality for bioconjugation and good electrochemical properties for signal transduction towards an all-polymer based biosensor. The CP-based interface was prepared by controlled co-polymerisation of 
EDOT and EDOT-COOH monomers using TBAP as a soft-template (Figure 6.4a), delivering the simultaneous formation of tunable 3D nanofibrous network and carboxylic acid groups (i.e. Nano-PEDOT-COOH). The ratio between EDOT and EDOT-COOH was evaluated to modulate the resulting nanostructure morphologies and density of the carboxylic acid groups.

The 3D nanofibrous network endowed the Nano-PEDOT-COOH interface with enhanced electron transfer and catalytic activity towards the electrooxidation of NADH, which can be ascribed to the relatively large 3D active surface area and porous structure. Furthermore, the carboxylic acid functionality provided anchoring sites for the stable immobilisation of a NADH-dependent dehydrogenase (i.e. lactate dehydrogenase) via EDC/S-NHS chemistry for the fabrication of a Bio-Nano-PEDOT-based biosensor for lactate detection that delivered a fast response and high sensitivity (Figure 6.4b). This strategy also provides a generic design for electrocatalytic devices based on the many available NADH-dependent dehydrogenases. 


\section{Tailoring PEDOT-based device interface for flexible bioelectronics: Research Paper V and Review Paper I}

\section{Research Paper V}

Conducting polymeric binder reinforced graphene network for 3D porous enzymatic electrodes and their application in wearable lactate biosensor

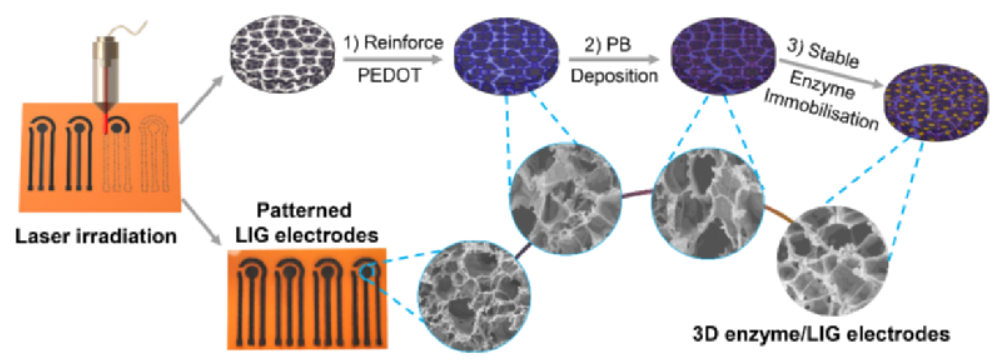

Figure 6.5 Schematic diagram of patternable LIG electrode system with 3D porous graphene structure for an enzymatic electrode in a flexible and wearable biosensor. 1) reinforcement of 3D porous graphene structure with PEDOT as polymeric binder; 2) nanoscale-coating of PB catalyst; 3) stable and effective immobilisation of enzyme into interconnected porous structure.

As discussed in Chapter 5, the trend in diagnostics nowadays is moving from conventional invasive $\mathrm{PoC}$ tests towards non-invasive flexible and wearable devices. The light-weight, flexible or conformable properties of flexible and wearable sensing devices can fulfil the requirements of mechanical behaviour for intimate interfacing with soft, deformable, and complex biological tissue with enhanced physical/biological event recognition and transduction. Despite the fact that various configurations have ingeniously exploited for flexible and wearable electrochemical sensors for physical and chemical signs monitoring, the implementation of these flexible and wearable sensors for electrochemical biosensing of biomarkers is extremely challenging owing to the need for biomolecular recognition elements layered on top of a transducer, for which purpose catalytic enzymes are most commonly applied.

In this paper, we demonstrate the development of a flexible and wearable platform for durable and sensitive detection of lactate. As shown in Figure 6.5, the flexible and wearable platform was based on the direct laser irradiation writing of honeycombstructured graphene network onto a conformable polyimide substrate. Subsequent polymerisation of PEDOT improved the mechanical stability as conductive polymeric 
binder by reinforcing the porous structure. Nano-deposited Prussian blue (PB) serves as the electrocatalyst. LOx enzyme was then stably and effectively immobilised into the pores as a 3D porous enzymatic electrode, and the open interconnected porous structure was well maintained. The resulting 3D enzymatic electrode system displayed excellent electrochemical biosensing of lactate with high sensitivity of $11.83 \mu \mathrm{A} \mathrm{mM}{ }^{-1}$ and selectivity due to the facilitated mass transport and charge transfer due to the specific $3 \mathrm{D}$ porous structure.

Further integration of LIG electrode into 3-electrode system realised a flexible and wearable platform for amperometric lactate biosensing in artificial sweat over a wide linear range of $0-16 \mathrm{mM}$ with a high sensitivity of $2.23 \mu \mathrm{A} \mathrm{mM}^{-1}$. The design and integration of the 3 -electrode system into conformable $2 \mathrm{D}$ patch and $3 \mathrm{D}$ wristband made no difference to the accurate detection of lactate. What is more, the dual-channel working electrode was designed and employed for simultaneous monitoring of glucose and lactate without any apparent cross-talk. Such an integrated flexible and wearable biosensing platform can be potentially expanded for multichannel and non-invasive monitoring of several physiological analytes in sweat, such as $\mathrm{pH}$, electrolyte, glucose, lactate and cortisol.

\section{Review Paper I}

Soft and flexible material-based affinity sensors

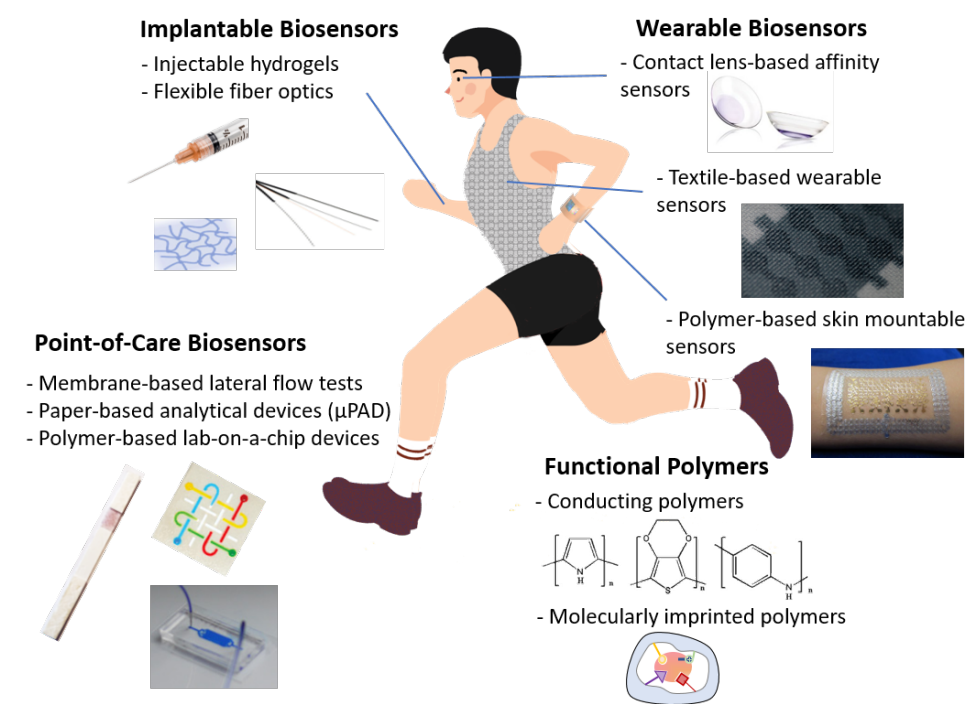

Figure 6.6 Illustration of soft and flexible materials employed for the development of affinity biosensors including PoC tests, wearable biosensors and implantable affinity biosensors. 
As discussed in Chapter 5 in this thesis, recent advances in biosensors and $\mathrm{PoC}$ devices are poised to change and expand the delivery of diagnostics from the conventional lateral-flow assays and test strips that dominate the market currently, to newly emerging wearable and implantable devices that can provide continuous monitoring. Soft and flexible materials, as discussed in Section 5.2, are playing a key role in propelling these trends towards real-time and remote health monitoring.

In this review paper, we tracked the evolution of affinity sensors from conventional lateral-flow test strips to wearable/implantable devices enabled by soft and flexible materials. Initially, we highlight the conventional affinity sensors exploiting membrane and paper materials which have been so successfully applied in PoC tests, such as lateral-flow immunoassay strips and emerging microfluidic paper-based devices. We then turn our attention to the multifarious polymer designs that provide both the base materials for sensor designs, such as PDMS, and more advanced functionalised materials that are capable of both recognition and transduction, such as conducting and molecularly imprinted polymers. The subsequent content discusses the wearable soft and flexible material-based affinity sensors, classified as flexible and skin-mountable, textile materials-based and contact lens-based affinity sensors. In the final sections, we explore the possibilities for implantable/injectable soft and flexible material-based affinity sensors, including hydrogels, microencapsulated sensors and optical fibers. The future importance of soft and flexible materials in affinity sensors for health management, therapy, food quality, environmental monitoring and research in the life sciences is clear and we need to bring chemists, engineers, users and entrepreneurs together to implement effective ways forward. 


\section{Chapter 7 Conclusion and Outlook}

This thesis demonstrates the tailoring of the CP-based interface with specific physicochemical characteristics for sensing and biosensing, such as positive/negative charge, micro/nano-structure and hybrid materials. The tailored CP-based interface served as a high-quality sensing interface through chemical signal transduction at the transducer interface, a biomolecule coupled bio-interface for specific analyte recognition, and a flexible and wearable bioelectronic device interface, delivering the enhanced analytical performance with improved sensitivity, selectivity and stability for sensing and biosensing.

The employment of CP-based interface for sensing and biosensing in this thesis for $\mathrm{NADH}, \mathrm{DA}$, lactate and glucose are based on the electrocatalytic or enzyme-coupled bioelectrocatalytic mechanism, which can be expanded to a wide range of biomarkers by immobilisation of biorecognition elements for affinity biosensing, such as antibody, nucleic acid, bacteria and so on. In addition to signal transduction based on electron transfer due to the electronic conductivity of CPs, further understanding and comparison of the sensing and biosensing mechanism arising from other effects (e.g. ionic conductivity changes, optical changes and conformable changes) are interesting to be elucidated. Additionally, further research in functionalisation of CPs with the aids of computer modelling such as COMSOL multiphysics for the rational design on the surface charge, morphologies and surface properties of CPs with optimal analytical performance, and minimized non-specific interactions with improved selectively.

By the combination of the excellent electrochemical performance of CPs as transducers and bio-interfaces demonstrated in this thesis with the soft polymeric substrates, CPs provide great opportunities for further development of all-polymer biosensors with unique mechanical flexibility and high biocompatibility, promoting the shift from classical metal and silicon to soft polymer electronics. The concept of all-polymer biosensors poses great challenges. The first consideration is the long-term stability and durability of bioreceptors. In addition, the exploration of CPs as other active components, such as conductors, power supplies and signal transmission processors, are all important. What is more, the mechanical compliance of $\mathrm{CP}$ active components to the polymeric substrates determines the attachment and stability of the all-polymer biosensors, since the large deformation (bending, twisting, and stretching, etc.) of such devices could potentially cause active component layer delamination from the electrode surface. 
Nowadays, much research interest has been devoted to the development of multiplex sensors that measure multiple physiological parameters ranging from physical signs (temperature, heart rate, body motion and strain etc.), to chemical-markers ( $\mathrm{pH}$, ions and humidity ect.) and to biomarkers (glucose, lactate and antibody etc.). The tailoring of CPs can help deliver a wide range of marker detection. The integration of physical, chemical and/or biological sensors are of vital importance for the development of advanced flexible and wearable bioelectronic devices with multiple functions. Soft and flexible materials will play a key role in the development of such devices, but development needs to keep pace and materials have to evolve from simple monofunctional components to composites and hybrid materials that integrate several of the required recognition, transduction and transmission functions to deliver more manufacturable and efficient sensor systems. 


\section{References}

1. W. H. Organization, World Health Organization. https://www.who.int/chp/about/integrated cd/en/, 2016.

2. A. P. Turner, Chem. Soc. Rev., 2013, 42, 3184-3196.

3. Y. Chen, Y.-S. Kim, B. W. Tillman, W.-H. Yeo and Y. Chun, Materials, 2018, 11, 522.

4. J. Li, W.-Y. Wong and X.-m. Tao, Nanoscale, 2020, 12, 1281-1306.

5. L. Meng, A. P. Turner and W. C. Mak, Biotechnol. Adv., 2020, 39, 107398.

6. S. Inal, J. Rivnay, A.-O. Suiu, G. G. Malliaras and I. McCulloch, Acc. Chem. Res., 2018, 51, 1368-1376.

7. D. Mawad, E. Stewart, D. L. Officer, T. Romeo, P. Wagner, K. Wagner and G. G. Wallace, Adv. Funct. Mater., 2012, 22, 2692-2699.

8. M. R. Abidian, K. A. Ludwig, T. C. Marzullo, D. C. Martin and D. R. Kipke, $A d v$. Mater., 2009, 21, 3764-3770.

9. M. R. Abidian, D. H. Kim and D. C. Martin, Adv. Mater., 2006, 18, 405-409.

10. R. Baughman, Synth. Met., 1996, 78, 339-353.

11. L. Meng, A. P. Turner and W. C. Mak, Biotechnol. Adv., 2019.

12. G. Walker, J. Ramsey, R. Cavin III, D. Herr, C. Merzbacher and V. Zhirnov, Natl. Inst. Stan., 2009.

13. H. A. Ledesma, X. Li, J. L. Carvalho-de-Souza, W. Wei, F. Bezanilla and B. Tian, Nat. Nanotechnol., 2019, 14, 645-657.

14. W. Gao, S. Emaminejad, H. Y. Y. Nyein, S. Challa, K. Chen, A. Peck, H. M. Fahad, H. Ota, H. Shiraki and D. Kiriya, Nature, 2016, 529, 509.

15. M. Rivera-Ruiz, C. Cajavilca and J. Varon, Tex. Heart I.J., 2008, 35, 174.

16. E. Neher and B. Sakmann, Sci. Am., 1992, 266, 44-51.

17. M. A. Lebedev and M. A. L. Nicolelis, Trends Neurosci., 2006, 29, 536-546.

18. Y. Fang, X. Li and Y. Fang, J. Mater. Chem. C, 2015, 3, 6424-6430.

19. A. H. B. Wu, Clin. Chim. Acta, 2006, 369, 119-124.

20. A. Sassolas, B. D. Leca-Bouvier and L. J. Blum, Chem. Rev., 2008, 108, 109-139.

21. S. Thakur, V. K. Thakur and O. A. Arotiba, in Hydrogels, Springer, 2018, pp. 2950 .

22. H. Yuk, B. Lu and X. Zhao, Chem. Soc. Rev., 2019, 48, 1642-1667.

23. A. Zhang and C. M. Lieber, Chem. Rev., 2016, 116, 215-257.

24. D. Berger, MLO Med. Lab. Obs., 1999, 31, 28-30.

25. A. Pavé, in Hierarchy in Natural and Social Sciences, Springer, 2006, ch. 2, pp. 3970.

26. H. K. Walker, W. D. Hall and J. W. Hurst, in Clinical Methods: The History, Physical, and Laboratory Examinations, Butterworths, 1990, ch. 217, pp. 987-989.

27. R. Mayeux, NeuroRx, 2004, 1, 182-188.

28. T. R. Walsh and M. R. Knecht, Chem. Rev., 2017, 117, 12641-12704.

29. M. Zhang, G. Qing and T. Sun, Chem. Soc. Rev., 2012, 41, 1972-1984.

30. T. Sun, G. Qing, B. Su and L. Jiang, Chem. Soc. Rev., 2011, 40, 2909-2921.

31. M. Berggren and A. Richter - Dahlfors, Adv. Mater., 2007, 19, 3201-3213.

32. H.-P. Loock and P. D. Wentzell, Sensor. Actuat. B-Chem, 2012, 173, 157-163. 
33. N. Elgrishi, K. J. Rountree, B. D. McCarthy, E. S. Rountree, T. T. Eisenhart and J. L. Dempsey, J. Chem. Educ., 2018, 95, 197-206.

34. F. R. Simões and M. G. Xavier, in Nanoscience and its Applications, William Andrew, 2016, ch. 6, pp. 155-178.

35. A. J. Bard, J. Chem. Educ., 1983, 60, 302.

36. E. E. Tanner and R. G. Compton, Electroanalysis, 2018, 30, 1336-1341.

37. A. Karyakin, in Electrochemical sensors, biosensors and their biomedical applications, Academic Press, 2008, ch. 13, pp. 411-439.

38. C. Gervais, M.-A. Languille, S. Reguer, M. Gillet, S. Pelletier, C. Garnier, E. P. Vicenzi and L. Bertrand, J. Anal. At. Spectrom., 2013, 28, 1600-1609.

39. X. Wang, N. Yang, Q. Wan and X. Wang, Sensor. Actuat. B-Chem, 2007, 128, 8390.

40. N. Vishnu, A. S. Kumar, G.-T. Pan and T. C. K. Yang, Sensor. Actuat. B-Chem, 2018, 275, 31-42.

41. H. Häkkinen, Nat. Chem., 2012, 4, 443.

42. J. Kochana, K. Starzec, M. Wieczorek, P. Knihnicki, M. Góra, A. Rokicińska, P. Kościelniak and P. Kuśtrowski, J. Solid State Electrochem., 2019, 23, 1463-1472.

43. S. R. Nxele, P. Mashazi and T. Nyokong, Electroanalysis, 2015, 27, 2468-2478.

44. F. S. Omar, N. Duraisamy, K. Ramesh and S. Ramesh, Biosens. Bioelectron., 2016, 79, 763-775.

45. S. A. Kumar and S.-M. Chen, Sensors, 2008, 8, 739-766.

46. Y. Shu, J. Xu, J. Chen, Q. Xu, X. Xiao, D. Jin, H. Pang and X. Hu, Sensor. Actuat. B-Chem, 2017, 252, 72-78.

47. W. Chen, S. Cai, Q.-Q. Ren, W. Wen and Y.-D. Zhao, Analyst, 2012, 137, 49-58.

48. H. Liu, L. Weng and C. Yang, Microchim. Acta, 2017, 184, 1267-1283.

49. S. B. Hall, E. A. Khudaish and A. L. Hart, Electrochim. Acta, 1998, 43, 2015-2024.

50. T. Q. Trung and N. E. Lee, Adv. Mater., 2016, 28, 4338-4372.

51. S. A. Piletsky and A. P. Turner, Electroanalysis, 2002, 14, 317-323.

52. E. J. Cho, J.-W. Lee and A. D. Ellington, Annu. Rev. Anal. Chem., 2009, 2, 241-264.

53. C. Zhu, G. Yang, H. Li, D. Du and Y. Lin, Anal. Chem., 2015, 87, 230-249.

54. L. Gorton, A. Lindgren, T. Larsson, F. Munteanu, T. Ruzgas and I. Gazaryan, Anal. Chim. Acta, 1999, 400, 91-108.

55. S. Shleev, J. Tkac, A. Christenson, T. Ruzgas, A. I. Yaropolov, J. W. Whittaker and L. Gorton, Biosens. Bioelectron., 2005, 20, 2517-2554.

56. A. K. Yagati, T. Lee, J. Min and J.-W. Choi, Bioelectrochemistry, 2011, 80, 169174.

57. Y. Ito, J. Okuda-Shimazaki, W. Tsugawa, N. Loew, I. Shitanda, C.-E. Lin, J. La Belle and K. Sode, Biosens. Bioelectron., 2019, 129, 189-197.

58. Y. Yamashita, I. Lee, N. Loew and K. Sode, Curr. Opin. Electrochem., 2018, 12, 92-100.

59. B. L. Treu and S. D. Minteer, Bioelectrochemistry, 2008, 74, 73-77.

60. A. Sassolas, L. J. Blum and B. D. Leca-Bouvier, Biotechnol. Adv., 2012, 30, 489511.

61. I. Mazurenko, K. Monsalve, P. Infossi, M.-T. Giudici-Orticoni, F. Topin, N. Mano and E. Lojou, Energ. Environ. Sci., 2017, 10, 1966-1982.

62. D. Sarauli, K. Peters, C. Xu, B. Schulz, D. Fattakhova-Rohlfing and F. Lisdat, ACS Appl. Mater. Inter., 2014, 6, 17887-17893. 
63. L. Shen, J. Ying, L. Ren, Y. Yao, Y. Lu, Y. Dong, G. Tian, X.-Y. Yang and B.-L. Su, J. Phys. Chem. Solids, 2019, 130, 1-5.

64. K. Rathee, V. Dhull, R. Dhull and S. Singh, Biochem. Biophys. Rep., 2016, 5, 3554.

65. L. Rassaei, W. Olthuis, S. Tsujimura, E. J. Sudhölter and A. van den Berg, Anal. Bioanal. Chem., 2014, 406, 123-137.

66. P. J. Derbyshire, H. Barr, F. Davis and S. P. Higson, J. Physiol. Sci., 2012, 62, 429440.

67. E. V. Karpova, A. I. Laptev, E. A. Andreev, E. E. Karyakina and A. A. Karyakin, ChemElectroChem, 2020, 7, 191-194.

68. J. Wang, Chem. Rev., 2008, 108, 814-825.

69. J. Kim, A. S. Campbell, B. E.-F. de Ávila and J. Wang, Nat. Biotechnol., 2019, 37, 389-406.

70. J. D. Newman and A. P. Turner, Biosens. Bioelectron., 2005, 20, 2435-2453.

71. S. Ferri, K. Kojima and K. Sode, J. Diabetes Sci. Technol., 2011.

72. J. Okuda-Shimazaki, H. Yoshida and K. Sode, Bioelectrochemistry, 2020, 132, 107414.

73. P. Chandrasekhar, Conducting polymers, fundamentals and applications: including carbon nanotubes and graphene, Springer, 2018.

74. S. C. Luo, H. h. Yu, A. C. Wan, Y. Han and J. Y. Ying, Small, 2008, 4, 2051-2058.

75. J. M. Pringle, M. Forsyth, D. R. MacFarlane, K. Wagner, S. B. Hall and D. L. Officer, Polymer, 2005, 46, 2047-2058.

76. T. K. Das and S. Prusty, Polymer-Plast. Technol., 2012, 51, 1487-1500.

77. J. G. Ibanez, M. E. Rincón, S. Gutierrez-Granados, M. h. Chahma, O. A. JaramilloQuintero and B. A. Frontana-Uribe, Chem. Rev., 2018, 118, 4731-4816.

78. T. Nezakati, A. Seifalian, A. Tan and A. M. Seifalian, Chem. Rev., 2018, 118, 67666843.

79. L. Groenendaal, F. Jonas, D. Freitag, H. Pielartzik and J. R. Reynolds, Adv. Mater., 2000, 12, 481-494.

80. S. A. Spanninga, D. C. Martin and Z. Chen, J. Phys. Chem. C, 2010, 114, 1499815004.

81. W. Shi, Q. Yao, S. Qu, H. Chen, T. Zhang and L. Chen, NPG Asia Mater., 2017, 9, e405-e405.

82. T. Goda, M. Toya, A. Matsumoto and Y. Miyahara, ACS Appl. Mater. Inter., 2015, 7, 27440-27448.

83. P. Chandrasekhar, in Conducting Polymers, Fundamentals and Applications, Springer, 2018, ch. 30, pp. 283-309.

84. W. Hai, T. Goda, H. Takeuchi, S. Yamaoka, Y. Horiguchi, A. Matsumoto and Y. Miyahara, ACS Appl. Mater. Inter., 2017, 9, 14162-14170.

85. B. Yu, J. Liu, S. Liu and F. Zhou, Chem. Commun., 2010, 46, 5900-5902.

86. K. Kwak, S. S. Kumar and D. Lee, Nanoscale, 2012, 4, 4240-4246.

87. A. Liu, M. D. Wei, I. Honma and H. Zhou, Adv. Funct. Mater., 2006, 16, 371-376.

88. A.-L. Sun, Y.-F. Zhang, G.-P. Sun, X.-N. Wang and D. Tang, Biosens. Bioelectron., 2017, 89, 659-665.

89. R. F. Vreeland, C. W. Atcherley, W. S. Russell, J. Y. Xie, D. Lu, N. D. Laude, F. Porreca and M. L. Heien, Anal. Chem., 2015, 87, 2600-2607.

90. L. Meng, A. P. F. Turner and W. C. Mak, Biosens. Bioelectron., 2020, 159, 112181. 
91. L. Zhang, Y. Wen, Y. Yao, J. Xu, X. Duan and G. Zhang, Electrochim. Acta, 2014, 116, 343-354.

92. N. F. Atta, A. Galal and R. A. Ahmed, Bioelectrochemistry, 2011, 80, 132-141.

93. V.-Q. Nguyen, D. Schaming, P. Martin and J.-C. Lacroix, ACS Appl. Mater. Inter., 2015, 7, 21673-21681.

94. J. Travas-Sejdic, N. Aydemir, B. Kannan, D. Williams and J. Malmström, J. Mater. Chem. B, 2014, 2, 4593-4609.

95. X. Zhang, J.-S. Lee, G. S. Lee, D.-K. Cha, M. J. Kim, D. J. Yang and S. K. Manohar, Macromolecules, 2006, 39, 470-472.

96. B. Dong, N. Lu, M. Zelsmann, N. Kehagias, H. Fuchs, C. M. Sotomayor Torres and L. F. Chi, Adv. Funct. Mater., 2006, 16, 1937-1942.

97. S. M. Fonseca, T. Moreira, A. J. Parola, C. Pinheiro and C. A. Laia, Sol. Energy Mater. Sol. Cells, 2017, 159, 94-101.

98. J.-W. Back, S. Lee, C.-R. Hwang, C.-S. Chi and J.-Y. Kim, Macromol. Res., 2011, 19, 33-37.

99. H. Yoon, M. Chang and J. Jang, Adv. Funct. Mater., 2007, 17, 431-436.

100. Y.-F. Lin, C.-T. Li and K.-C. Ho, J. Mater. Chem. A, 2016, 4, 384-394.

101. L. Xia, Z. Wei and M. Wan, J. Colloid Interface Sci., 2010, 341, 1-11.

102. S. C. Luo, J. Sekine, B. Zhu, H. C. Zhao, A. Nakao and H. H. Yu, Acs Nano, 2012, 6, 3018-3026.

103. E. Poverenov, M. Li, A. Bitler and M. Bendikov, Chem. Mater., 2010, 22, 40194025.

104. N. Liu, G. Fang, J. Wan, H. Zhou, H. Long and X. Zhao, J. Mater. Chem., 2011, 21, 18962-18966.

105. C. L. Choong, M. B. Shim, B. S. Lee, S. Jeon, D. S. Ko, T. H. Kang, J. Bae, S. H. Lee, K. E. Byun and J. Im, Adv. Mater., 2014, 26, 3451-3458.

106. S. J. Wilks, S. M. Richardson-Burn, J. L. Hendricks, D. Martin and K. J. Otto, Front. Neuroeng., 2009, $2,7$.

107. D. Mantione, I. del Agua, A. Sanchez-Sanchez and D. Mecerreyes, Polymers, 2017, 9, 354.

108. Y. Yao, Y. Wen, L. Zhang, Z. Wang, H. Zhang and J. Xu, Anal. Chim. Acta, 2014, 831, 38-49.

109. S. Macher, M. Schott, M. Sassi, I. Facchinetti, R. Ruffo, G. Patriarca, L. Beverina, U. Posset, G. A. Giffin and P. Löbmann, Adv. Funct. Mater., 2020, 30, 1906254.

110. I. Schwendeman, C. L. Gaupp, J. M. Hancock, L. Groenendaal and J. R. Reynolds, Adv. Funct. Mater., 2003, 13, 541-547.

111. C. A. Cutler, M. Bouguettaya, T.-S. Kang and J. R. Reynolds, Macromolecules, 2005, 38, 3068-3074.

112. H. Zhao, B. Zhu, S.-C. Luo, H.-A. Lin, A. Nakao, Y. Yamashita and H.-h. Yu, ACS Appl. Mater. Inter., 2013, 5, 4536-4543.

113. S.-C. Luo, Polym. Rev., 2013, 53, 303-310.

114. C. H. Chen and S. C. Luo, ACS Appl Mater Interfaces, 2015, 7, 21931-21938.

115. J. Sekine, S. C. Luo, S. Wang, B. Zhu, H. R. Tseng and H. h. Yu, Adv. Mater., 2011, 23, 4788-4792.

116. S.-C. Luo, E. Mohamed Ali, N. C. Tansil, H.-h. Yu, S. Gao, E. A. Kantchev and J. Y. Ying, Langmuir, 2008, 24, 8071-8077. 
117. E. M. Ali, E. A. B. Kantchev, H.-h. Yu and J. Y. Ying, Macromolecules, 2007, 40, 6025-6027.

118. S.-C. Luo, H. Xie, N. Chen and H.-h. Yu, ACS Appl. Mater. Inter., 2009, 1, 14141419.

119. Y. Wang, S. Bai, L. Cheng, N. Wang, J. Wang, F. Gao and W. Huang, Adv. Mater., 2016, 28, 4532-4540.

120. K. Sun, S. Zhang, P. Li, Y. Xia, X. Zhang, D. Du, F. H. Isikgor and J. Ouyang, J. Mater. Sci.: Mater. Electron., 2015, 26, 4438-4462.

121. J. Wang, G. Cai, X. Zhu and X. Zhou, J. Appl. Polym. Sci., 2012, 124, 109-115.

122. S. Kirchmeyer and K. Reuter, J. Mater. Chem., 2005, 15, 2077-2088.

123. R. Singh, J. Tharion, S. Murugan and A. Kumar, ACS Appl. Mater. Inter., 2017, 9, 19427-19435.

124. S.-M. Kim, C.-H. Kim, Y. Kim, N. Kim, W.-J. Lee, E.-H. Lee, D. Kim, S. Park, K. Lee and J. Rivnay, Nat. Commun., 2018, 9, 3858.

125. Y. Hui, C. Bian, S. Xia, J. Tong and J. Wang, Anal. Chim. Acta, 2018, 1022, 1-19. 126. R. Temmer, A. Maziz, C. Plesse, A. Aabloo, F. Vidal and T. Tamm, Smart Mater. Struct., 2013, 22, 104006.

127. F. Pires, Q. Ferreira, C. A. Rodrigues, J. Morgado and F. C. Ferreira, BBA-Gen. Subjects, 2015, 1850, 1158-1168.

128. D. W. Hatchett and M. Josowicz, Chem. Rev., 2008, 108, 746-769.

129. T.-Y. Huang, C.-W. Kung, H.-Y. Wei, K. M. Boopathi, C.-W. Chu and K.-C. Ho, J. Mater. Chem. A, 2014, 2, 7229-7237.

130. G. Xu, B. Li, X. T. Cui, L. Ling and X. Luo, Sensor. Actuat. B-Chem, 2013, 188, 405-410.

131. A. Balamurugan, K.-C. Ho, S.-M. Chen and T.-Y. Huang, Colloid. Surface. A, 2010, 362, 1-7.

132. J. Wang, Y. Wang, M. Cui, S. Xu and X. Luo, Microchim. Acta, 2017, 184, 483489.

133. A. Kros, S. W. van Hövell, N. A. Sommerdijk and R. J. Nolte, Adv. Mater., 2001, 13, $1555-1557$.

134. H. Zheng, S. Zhang, X. Liu, Y. Zhou and S. Alwarappan, Biosens. Bioelectron., 2020, 162, 112234.

135. D. Li, M. B. Müller, S. Gilje, R. B. Kaner and G. G. Wallace, Nat. Nanotechnol., 2008, 3, 101.

136. W. Si, W. Lei, Z. Han, Y. Zhang, Q. Hao and M. Xia, Sensor. Actuat. B-Chem, 2014, 193, 823-829.

137. I. M. Taylor, E. M. Robbins, K. A. Catt, P. A. Cody, C. L. Happe and X. T. Cui, Biosens. Bioelectron., 2017, 89, 400-410.

138. M. Xu, D. Obodo and V. K. Yadavalli, Biosens. Bioelectron., 2019, 124, 96-114.

139. G. Yao, C. Yin, Q. Wang, T. Zhang, S. Chen, C. Lu, K. Zhao, W. Xu, T. Pan and M. Gao, J. Materiomics, 2020, 6, 397-413.

140. Y. Yang, X. Yang, Y. Tan and Q. Yuan, Nano Res., 2017, 10, 1560-1583.

141. J. R. Sempionatto, T. Nakagawa, A. Pavinatto, S. T. Mensah, S. Imani, P. Mercier and J. Wang, Lab Chip, 2017, 17, 1834-1842.

142. J. Park, J. Kim, S.-Y. Kim, W. H. Cheong, J. Jang, Y.-G. Park, K. Na, Y.-T. Kim, J. H. Heo and C. Y. Lee, Sci. Adv., 2018, 4, eaap9841. 
143. M. S. Mannoor, H. Tao, J. D. Clayton, A. Sengupta, D. L. Kaplan, R. R. Naik, N. Verma, F. G. Omenetto and M. C. McAlpine, Nat. Commun., 2012, 3, 763.

144. H. Lee, T. K. Choi, Y. B. Lee, H. R. Cho, R. Ghaffari, L. Wang, H. J. Choi, T. D. Chung, N. Lu and T. Hyeon, Nat. Nanotechnol., 2016, 11, 566-572.

145. D.-H. Kim, N. Lu, R. Ghaffari, Y.-S. Kim, S. P. Lee, L. Xu, J. Wu, R.-H. Kim, J. Song and Z. Liu, Nat. Mater., 2011, 10, 316-323.

146. K. Tybrandt, D. Khodagholy, B. Dielacher, F. Stauffer, A. F. Renz, G. Buzsáki and J. Vörös, Adv. Mater., 2018, 30, 1706520.

147. T. Yamada, Y. Hayamizu, Y. Yamamoto, Y. Yomogida, A. Izadi-Najafabadi, D. N. Futaba and K. Hata, Nat. Nanotechnol., 2011, 6, 296.

148. Y. Chen, Y. Zhang, Z. Liang, Y. Cao, Z. Han and X. Feng, npj Flex. Electron., 2020, 4, 1-20.

149. H. Lee, C. Song, Y. S. Hong, M. S. Kim, H. R. Cho, T. Kang, K. Shin, S. H. Choi, T. Hyeon and D.-H. Kim, Sci. Adv., 2017, 3, e1601314.

150. J. C. Costa, F. Spina, P. Lugoda, L. Garcia-Garcia, D. Roggen and N. Münzenrieder, Technologies, 2019, 7, 35.

151. S. Xu, Y. Zhang, L. Jia, K. E. Mathewson, K.-I. Jang, J. Kim, H. Fu, X. Huang, P. Chava and R. Wang, Science, 2014, 344, 70-74.

152. J. Xiao, Y. Liu, L. Su, D. Zhao, L. Zhao and X. Zhang, Anal. Chem., 2019, 91, 14803-14807.

153. A. Martín, J. Kim, J. F. Kurniawan, J. R. Sempionatto, J. R. Moreto, G. Tang, A. S. Campbell, A. Shin, M. Y. Lee and X. Liu, ACS sensors, 2017, 2, 1860-1868.

154. H. Lin, Y. Zhao, S. Lin, B. Wang, C. Yeung, X. Cheng, Z. Wang, T. Cai, W. Yu and K. King, Lab Chip, 2019, 19, 2844-2853.

155. T. Someya, Z. Bao and G. G. Malliaras, Nature, 2016, 540, 379-385.

156. I. Gualandi, E. Scavetta, F. Mariani, D. Tonelli, M. Tessarolo and B. Fraboni, Electrochim. Acta, 2018, 268, 476-483.

157. L. Basiricò, P. Cosseddu, A. Scidà, B. Fraboni, G. G. Malliaras and A. Bonfiglio, Org. Electron., 2012, 13, 244-248.

158. K. Kiilerich-Pedersen, C. R. Poulsen, T. Jain and N. Rozlosnik, Biosens. Bioelectron., 2011, 28, 386-392.

159. A. Blau, A. Murr, S. Wolff, E. Sernagor, P. Medini, G. Iurilli, C. Ziegler and F. Benfenati, Biomaterials, 2011, 32, 1778-1786.

160. J. Dapra, L. H. Lauridsen, A. T. Nielsen and N. Rozlosnik, Biosens. Bioelectron., 2013, 43, 315-320.

161. J. Kirkegaard and N. Rozlosnik, in Biosensors and Biodetection, Springer, 2017, ch. 5 , pp. 55-70.

162. E. Bihar, S. Wustoni, A. M. Pappa, K. N. Salama, D. Baran and S. Inal, npj Flex. Electron., 2018, 2, 1-8. 


\section{Papers}

The papers associated with this thesis have been removed for copyright reasons. For more details about these see:

http://urn.kb.se/resolve?urn=urn:nbn:se:liu:diva-169499 


\section{FACULTY OF SCIENCE AND ENGINEERING}

Linköping Studies in Science and Technology, Dissertation No. 2094, 2020

Department of Physics, Chemistry and Biology

Linköping University

SE-581 83 Linköping, Sweden

www.liu.se 